\title{
Centromere identity is dependent on nuclear spatial organization
}

\author{
Weifang $\mathrm{Wu}^{1,2}$, Toni McHugh ${ }^{1,2}$, David A. Kelly ${ }^{1,2}$, Alison L. Pidoux ${ }^{1,2}$ \\ and Robin C. Allshire ${ }^{1,2, *}$
}

${ }^{1}$ Wellcome Trust Centre for Cell Biology, ${ }^{2}$ Institute of Cell Biology,

School of Biological Sciences, The University of Edinburgh,

Edinburgh EH9 3BF, Scotland UK.

* Corresponding author: robin.allshire@ed.ac.uk

Tel: 00-44-7753-741307 


\section{Summary}

The establishment of centromere-specific CENP-A chromatin is influenced by epigenetic and genetic processes. Central domain sequences from fission yeast centromeres are preferred substrates for CENP-A ${ }^{\text {Cnp1 }}$ incorporation, but their use is context dependent, requiring adjacent heterochromatin. CENP-A ${ }^{\text {Cnp1 }}$ overexpression bypasses heterochromatin dependency, suggesting heterochromatin ensures exposure to conditions or locations permissive for CENP-A ${ }^{C n p 1}$ assembly. Centromeres cluster around spindle-pole bodies (SPBs). We show that heterochromatin-bearing minichromosomes localize close to SPBs, consistent with this location promoting CENP-A ${ }^{\text {Cnp1 }}$ incorporation. We demonstrate that heterochromatin-independent de novo CENP-A ${ }^{\text {Cnp1 }}$ chromatin assembly occurs when central domain DNA is placed near, but not far from, endogenous centromeres or neocentromeres. Moreover, direct tethering of central domain DNA at SPBs permits CENP-A ${ }^{\text {Cnp1 }}$ assembly, suggesting that the nuclear compartment surrounding SPBs is permissive for CENP-A ${ }^{\text {Cnp1 }}$ incorporation because target sequences are exposed to high levels of CENP-A ${ }^{\text {Cnp1 }}$ and associated assembly factors. Thus, nuclear spatial organization is a key epigenetic factor that influences centromere identity.

Keywords: Centromere identity, CENP-A, Spindle Pole Body, heterochromatin, spatial organization, fission yeast, S. pombe. 


\section{Introduction}

Centromeres are specialized chromosomal sites where multiprotein complexes known as kinetochores are assembled. Kinetochores attach chromosomes to spindle microtubules to mediate accurate mitotic and meiotic chromosome segregation. The assembly of kinetochores in many eukaryotes including yeasts and humans relies on specialized centromeric chromatin in which canonical histone $\mathrm{H} 3$ is replaced by the CENP-A/cenH3 histone $\mathrm{H} 3$ variant (Cnp1 in fission yeast, Schizosaccharomyces pombe). CENP-A-containing chromatin provides the underlying epigenetic mark that specifies the chromosomal site at which kinetochores assemble. CENP-A is required to establish and maintain centromere identity and thus indicates active centromeres (Allshire and Karpen, 2008; Mellone and Fachinetti, 2021).

In organisms with monocentric chromosomes centromeres are confined to a single locus on each chromosome. Such centromeres are often composed of long tandem arrays of repetitive sequences such as $\alpha$-satellite repeats on human chromosomes (DeBose-Scarlett and Sullivan, 2021). These repeats provide a substrate for the de novo establishment of CENP-A chromatin and assembly of functional kinetochores when introduced into human cells. Thus, $\alpha$-satellite repeats trigger centromere formation. Acentric chromosomes lacking centromeres are unable to attach to spindle microtubules and are lost during cell division. However, following centromere ablation through centromere inactivation or deletion of centromere DNA, neocentromeres can arise spontaneously at unusual locations that lack sequence similarity to normal centromere DNA but allow stable segregation of such acentric chromosomes (DeBose-Scarlett and Sullivan, 2021; Ishii et al., 2008). Thus, centromeric DNAs are not the only sequences that can trigger the assembly of functional kinetochores. Once assembled at a particular location, including neocentromeres or sites that do not usually incorporate CENP-A, CENP-A chromatin is stably propagated at that site though cell division using intrinsic maintenance mechanisms (Mitra et al., 2020; Westhorpe and Straight, 2015). Consequently, prior CENP-A assembly can mark a chromosomal locus for continued persistence of CENP-A chromatin on one homologue whereas the same locus remains devoid of CENP-A on the other (DeBose-Scarlett and Sullivan, 2021).

The fission yeast genome is carried on three monocentric chromosomes with regional centromeres of 40-110 kb comprised of two distinct domains (see Figure S1): CENP-A ${ }^{\text {Cnp1 }}$ chromatin assembles 
across the central domain consisting of central core $(c c)$ and flanking innermost repeat (imr) DNA, which are surrounded outer repeats (otr-dg/dh) assembled in Clr4 Histone H3 lysine 9 methyl(H3K9me)-transferase-dependent heterochromatin (Allshire and Ekwall, 2015; Martienssen and Moazed, 2015). The central core of centromere 2 (cc2) is unique but the central cores of cen1 and cen3 share the same sequence. imr elements are unique to each centromere and mark the transition between CENP-A ${ }^{\text {Cnp1 }}$ chromatin and the heterochromatic otr-dg/dh repeats which are conserved in sequence, but not arrangement, between the three centromeres (Clarke et al., 1993; Takahashi et al., 1992). tRNA genes that reside in each imr element demarcate these distinct centromeric domains and prevent heterochromatin from encroaching into the central CENP-A ${ }^{\text {Cnp1 }}$ chromatin domain (Noma et al., 2006; Scott et al., 2006). Two divergent Schizosaccharomyces species (S. octosporus and S. cryophilus) share a similar centromere domain organization (Tong et al., 2019).

Like human $\alpha$-satellite centromeric DNA, fission yeast central domain DNA is a preferred substrate for CENP-A ${ }^{\text {Cnp1 }}$ and kinetochore assembly. This preferred status is underscored by the observation that, in contrast to other sequences, naïve central domain DNA borne on minichromosomes readily assembles and maintains CENP-A ${ }^{\text {Cnp1 }}$ chromatin following transient CENP-A ${ }^{\text {Cnp1 }}$ overexpression, bypassing the usual requirement for adjacent heterochromatin (Castillo et al., 2013; Catania et al., 2015). Interestingly, despite having no sequence homology with S. pombe centromeres, central domains from S. octosporus and S. cryophilus are competent to assemble CENP-A ${ }^{\text {Cnp1 }}$ chromatin and functional centromeres in S. pombe, indicating that fission yeast central domains possess conserved instructive features (Tong et al., 2019). S. pombe central domain sequences are transcribed by RNAPII and exhibit high rates of histone $\mathrm{H} 3$ turnover which may contribute to the replacement of $S$ phase-deposited placeholder $H 3$ with CENP-A ${ }^{\mathrm{Cnp} 1}$ during the subsequent $\mathrm{G} 2$ (Shukla et al., 2018; Singh et al., 2020). H3 is evicted from central domain chromatin even in the absence of CENP-A and kinetochore proteins (Shukla et al., 2018). The Mis18 complex acts in concert with the CENP-A chaperone HJURP to recognize pre-existing CENP-A nucleosomes and ensure their persistence at particular locations by mediating $\mathrm{H} 3$ replacement with CENP-A in new H3-containing nucleosomes assembled during the preceding S-phase (Mitra et al., 2020; Westhorpe and Straight, 2015; Zasadzińska and Foltz, 2017). Thus, fission yeast central domain DNA possesses innate sequence-driven properties that program H3 eviction, making it a favored substrate for CENP-A $\mathrm{An}^{\mathrm{Cnp}}$ chromatin assembly, which, once assembled is rendered heritable though 
an intricate read-write mechanism.

Centromeres are tightly clustered around spindle pole bodies (SPBs; centrosome equivalents) during interphase in both fission (S. pombe) and budding (Saccharomyces cerevisiae) yeast (Funabiki et al., 1993; Muller et al., 2019; Winey and O'Toole, 2001). In S. cerevisiae SPB-tocentromere microtubules persist in G1 and mediate SPB-centromere clustering (Guacci et al., 1997; Jaspersen, 2021; Winey and O'Toole, 2001). Proper centromere clustering around S. pombe SPBs is dependent on the functions of SPB component Sad1 (LINC complex SUN domain protein) and Lem2 (LEM domain inner nuclear membrane protein) which is distributed around the entire nuclear envelope (NE) but is concentrated at SPBs (Ebrahimi et al., 2018; Fernández-Álvarez et al., 2016). Csi1, resides at the kinetochore-SPB interface and is required for Lem2 accumulation around SPBs and acts with Lem2 to maintain SPB-centromere associations (Barrales et al., 2016; Ebrahimi et al.,

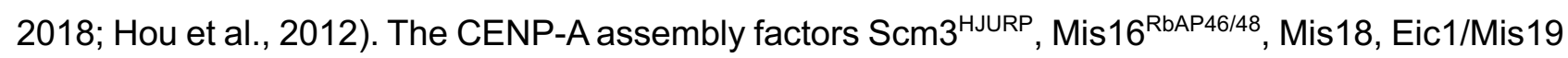
are concentrated at centromeres clustered close to SPBs from late anaphase to prophase, including during G2 when new CENP-A ${ }^{\text {Cnp1 }}$ is incorporated (Hayashi et al., 2004; Pidoux et al., 2009; Shukla et al., 2018; Subramanian et al., 2014; Williams et al., 2009).

Although fission yeast centromeric central domains are the preferred substrate for CENP-A ${ }^{\text {Cnp1 }}$ assembly, establishment of CENP-A ${ }^{\mathrm{Cnp} 1}$ chromatin is subject to epigenetic regulation. The de novo assembly of CENP-A $A^{\text {Cnp1 }}$ chromatin and functional centromeres on central domain sequences is dependent on the presence of adjacent outer repeat heterochromatin (see Figure S2) (Folco et al., 2008; Steiner and Clarke, 1994). Direct transformation of naked minichromosomes into cells lacking heterochromatin compared to crossing preassembled minichromosomes from wild-type cells results in a different fate: in the former central domain is assembled in H3 chromatin, in the latter it is assembled in CENP-A ${ }^{\text {Cnp1 }}$ chromatin (see Figure S2B, C) (Folco et al., 2008). These observations indicate that both context and prior history are important for determining chromatin state. Synthetic heterochromatin, assembled by tethering the Clr4 H3K9-methyltransferase, substitutes for outer repeats in promoting CENP-A ${ }^{\mathrm{Cnp} 1}$ assembly on minichromosomes when placed next to central domain DNA (see Figure S2F) (Kagansky et al., 2009). Thus, the properties of adjacent heterochromatin itself rather than other features of outer repeat elements, are critical for de novo CENP-A ${ }^{\text {Cnp1 }}$ assembly. Heterochromatin could promote the establishment of CENP-A ${ }^{\text {Cnp1 }}$ chromatin 
by recruitment of chromatin modifiers that influence turnover or other properties of histone $\mathrm{H} 3$ chromatin on adjacent central domain to favor CENP-A ${ }^{\text {Cnp1 }}$ deposition (Catania et al., 2015; Shukla et al., 2018). Alternatively, since CENP-A $A^{C n p 1}$ overexpression circumvents the need for flanking heterochromatin in such CENP-A ${ }^{\text {Cnp1 }}$ chromatin establishment assays (Catania et al., 2015) (see Figure S2) and endogenous heterochromatin domains are located at the nuclear periphery (Alfredsson-Timmins et al., 2007; Funabiki et al., 1993; Pichugina et al., 2016), it is possible that centromeric heterochromatin places such minichromosomes at a nuclear location that encourages de novo CENP-A ${ }^{\text {Cnp1 }}$ chromatin assembly. The centromere clusters at $S$. pombe SPBs would be expected to provide a compartment naturally enriched with CENP-A ${ }^{\mathrm{Cnp} 1}$ and its loading factors.

Here we test if the positioning of centromeric DNA relative to existing centromeres and/or SPBs influences de novo CENP-A ${ }^{\text {Cnp1 }}$ chromatin assembly and recruitment of kinetochore proteins. Heterochromatin-bearing plasmids localize close to SPBs, suggesting that heterochromatin may play a positioning role in promoting establishment of CENP-A ${ }^{\text {Cnp1 }}$ chromatin. We demonstrate that potentially functional centromeric central domain DNA does not assemble CENP-A ${ }^{\text {Cnp1 }}$ or kinetochore proteins unless inserted close to an already functional native centromere or neocentromere. Thus, proximity to an existing centromere in cis on the same chromosome promotes CENP-A ${ }^{\text {Cnp1 }}$ and kinetochore assembly. Direct tethering of naïve minichromosome-borne central domain DNA to SPB-associated proteins in the absence of flanking heterochromatin revealed that proximity in trans to SPB-centromere clusters is also sufficient to trigger assembly of CENP-A ${ }^{\text {Cnp1 }}$ chromatin and recruitment of kinetochore components. Thus, we define a key role for spatial genome organization, in particular centromere clustering, and resulting nuclear compartmentalization in determining centromere identity. Our findings reveal that centromeric heterochromatin functions to position centromeres within a nuclear compartment that ensures de novo CENP-A ${ }^{\text {Cnp1 }}$ chromatin assembly. 


\section{Results}

\section{Centromeric heterochromatin mediates association with the SPB-centromere cluster}

The de novo assembly of CENP-A ${ }^{\text {Cnp1 }}$ chromatin on naïve centromeric central domain DNA freshly introduced into fission yeast as DNA by transformation on plasmid-based minichromosomes requires H3K9me-dependent heterochromatin formation on flanking outer $d g / d h(K / L)$ centromere repeat DNA (Figure S2A, B and D) (Folco et al., 2008). Heterochromatin may influence CENP-A ${ }^{\text {Cnp1 }}$ chromatin establishment through nuclear positioning cues. To test if centromeric heterochromatin promotes association with SPBs we utilized autonomously replicating minichromosomes which are less constrained than endogenous chromosomal regions with respect to their positioning within nuclei. In all strains used, $6 \mathrm{~kb}$ of endogenous $c c 2$ was replaced with $5.5 \mathrm{~kb}$ of cen 1 central domain DNA (cc2A::cc1; Figure S1B). Thus, cc2 DNA carried by minichromosomes are unique sequences in these strains, allowing their specific analysis by quantitative chromatin immunoprecipitation ( $q$ ChIP). As a consequence of this manipulation, sequences common to wild-type $c c 1$ and $c c 3$ are present at all three endogenous centromeres in $c c 2 \Delta:: c c 1$ cells and provide a positive control comparator for CENP-A ${ }^{\mathrm{Cnp} 1}$ and kinetochore protein association. The pHet minichromosome carries outer repeat DNA (K”, $2 \mathrm{~kb}$ ) that is sufficient to trigger Clr4-dependent de novo heterochromatin formation when transformed into wild-type, but not clr4 $\Delta$, cells (Figure 1A, B; Table S2) (Folco et al., 2008; Kagansky et al., 2009). pcc2 carries 8.6 kb of cen2 central domain DNA but lacks outer repeat DNA (Figure 1A; Table S2) and thus cannot form heterochromatin and does not assemble CENP$A^{\text {Cnp1 }}$ chromatin or kinetochores (Figure 1C; Figure S2D) (Catania et al., 2015; Folco et al., 2008). However, pHcc2, carrying both outer repeat and cc2 DNA (Figure 1A; Table S2) forms heterochromatin which permits CENP-A ${ }^{\mathrm{Cnp} 1}$ chromatin (Figure $1 \mathrm{C}, \mathrm{D}$ ), kinetochores and functional centromeres to be frequently established in wild-type cells following transformation (Figure S2A, Figure S3) (Catania et al., 2015; Folco et al., 2008).

Fluorescent In Situ Hybridization (FISH) to the backbone plasmid and/or cc2 sequences allowed pHet, pcc2 or pHcc2 minichromosome localization in wild-type cells relative to SPBs (Cdc11, SPBspecific centriolin ortholog; Figure 1A). pcc2 was found at, or in close proximity to, SPBs in $17 \%$ of cells, however, the presence of a heterochromatic repeat on $\mathrm{pHcc} 2$ with resulting CENP-A ${ }^{\mathrm{Cnp} 1}$ and kinetochore assembly increased SPB association to $100 \%$ (Figure 1E, F). Consistent with a 
requirement for heterochromatin for SPB association, only low levels of pHcc2-SPB association were

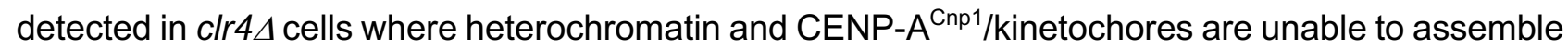
(Figures 1C-F; S3A). Moreover, pHet, which only assembles heterochromatin, localized close to SPBs in $73 \%$ of wild type cells but only $13 \%$ of clr4 $\Delta$ cells (Figure $1 \mathrm{~B}$, E and F).

Together these data indicate that centromeric outer repeat-induced heterochromatin is sufficient to mediate frequent contact with SPBs where centromeres and CENP-A ${ }^{\text {Cnp1 }}$ assembly factors are concentrated. Thus, we propose that centromeric heterochromatin promotes exposure of adjacent cc2 centromere DNA to this CENP-A ${ }^{\text {Cnp1 }}$ assembly factor-rich nuclear compartment, thereby ensuring the assembly of CENP-A ${ }^{C n p 1}$ chromatin and kinetochores.

\section{Centromeric central domain DNA assembles CENP-A ${ }^{\text {Cnp1 }}$ chromatin when inserted close to native centromeres}

To test if a nuclear compartment formed by SPB-centromere clustering might stimulate de novo CENP-A ${ }^{\text {Cnp } 1}$ chromatin assembly we inserted $8.6 \mathrm{~kb}$ of $c c 2$ DNA near or far from cen 1 and assayed for the presence of CENP-A ${ }^{\text {Cnp1 }}$ chromatin. In all strains used endogenous $c c 2$ had been replaced with $c c 1$ so that regions $L-t o-Q$ of the resulting $8.6 \mathrm{~kb} c c 2$ insertions are unique (cc2 $:: . c c 1$; Figure $\mathrm{S1B})$. The lys1 locus resides just $26 \mathrm{~kb}$ from $c c 1,11.3 \mathrm{~kb}$ from the left otr1 heterochromatin repeat while ade 3 is a distant 2,438 $\mathrm{kb}$ from cc1 (Figure 2A). Microscopy measurements demonstrated that lys1 and ade3 decorated with Lacl-GFP on lacO-array insertions (Ding and Hiraoka, 2017) are positioned in close proximity to or distant from SPBs, respectively, in three dimensional nuclear space (Figure 2B, C). qChIP analysis showed that CENP-A ${ }^{\text {Cnp1 }}$ was uniformly incorporated onto regions L-P across $c c 2$ following insertion at lys1 (lys1:cc2). In contrast, no CENP-A ${ }^{\text {Cnp1 }}$ enrichment was observed on cc2 inserted at ade3 (ade3:cc2) (Figure 2D). In addition, kinetochore proteins CENP-C ${ }^{\mathrm{Cnp} 3}$, CENP-K $\mathrm{K}^{\mathrm{Sim} 4}$ and $\mathrm{Knl} 1^{\mathrm{Spc7}}$ were also recruited to lys 1:cc2 (Figure 2E-G), indicating that CENP-A ${ }^{\text {Cnp1 }}$ deposition on lys 1:cc2 results in recruitment of both inner and outer kinetochore proteins. CENP-A ${ }^{C n p 1}$ was also incorporated on $c c 2$ inserted at $s d h 1,24 \mathrm{~kb}$ to the right of cen1-cc1, or at a location we named itg10, $27 \mathrm{~kb}$ from on the right side of cen2-cc2 $\Delta:: c c 1$ (itg10:cc2; Figures S4A, B). Insertion of $c c 2$ at locations $41 \mathrm{~kb}$ (vps29:cc2) and $47 \mathrm{~kb}$ (bud6:cc2) further away on the left side

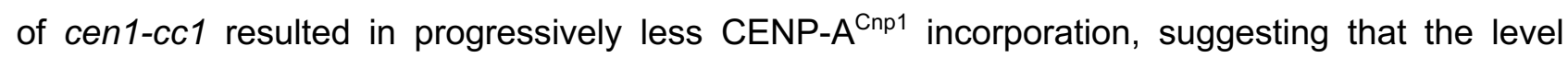
incorporated on inserted cc2 DNA is dependent on its proximity in cis to functional cen1 (Figure S4C). 
Thus, either proximity to an endogenous centromere in cis on the same chromosome, or exposure to a distinct nuclear compartment formed by SPB-centromere clusters, effectively mediates de novo CENP-A ${ }^{\text {Cnp1 }}$ assembly and kinetochore protein recruitment on naïve central domain DNA. cc2 DNA inserted close to cen 1 might acquire CENP-A ${ }^{\mathrm{Cnp} 1}$ chromatin as a result of it spreading from cen1 into lys1:cc2. However, little or no CENP-A ${ }^{\text {Cnp1 }}$ enrichment was detected at three positions (i-iii) between cen1 and lys1:cc2 (Figure S4D). Thus CENP-A ${ }^{\text {Cnp1 }}$ does not uniformly spread along the chromosome from its normal location at cen1-cc1 into the lys1:cc2 insert.

These analyses demonstrate that $c c 2$ DNA, a known substrate for fission yeast CENP-A ${ }^{\text {Cnp1 }}$ and kinetochore assembly, incorporates CENP-A ${ }^{\mathrm{Cnp} 1}$ when inserted in cis close to native centromeres. The finding that the levels of CENP-A ${ }^{\text {Cnp1 }}$ incorporated decrease with increasing distance from a centromere suggests that proximity to native centromeres provides an environment that is more favorable for CENP-A ${ }^{\text {Cnp1 }}$ and kinetochore assembly on naïve centromere DNA.

\section{Proximity to functional centromeres, not locus-specific context, promotes CENP-A ${ }^{\text {Cnp1 }}$ chromatin establishment}

Neocentromeres form near fission yeast telomeres when an endogenous centromere is deleted (Ishii et al., 2008) (Figure 3A). Deletion of cen1 (cen1A) results in neocentromeres being formed over the left (neo1L; cd39) or right (neo1R; cd60) subtelomeric regions on chromosome I (Ishii et al., 2008). FISH demonstrates that prior to neocentromere formation the subtelomeric neo1R locus is not located near SPBs whereas upon CENP-A ${ }^{\mathrm{Cnp} 1}$ assembly and neocentromere formation neo1R joins the interphase SPB-centromere cluster in $94 \%$ of cells, where CENP-A is concentrated (Figure 3AE). Unlike when $c c 2$ was inserted at lys1 in cells with a nearby functional cen1 (Figure 2), insertion of $c c 2$ at lys 1 in cen $1 \Delta$ cells with the neo1R neocentromere $1.8 \mathrm{Mb}$ away failed to incorporate CENP$A^{C n p 1}$ (Figure 4A, B). This finding suggests that CENP-A ${ }^{C n p 1}$ fails to be incorporated at lys1:cc2 upon insertion in cells with this neocentromere because lys 1 is displaced from the centromere cluster. Thus, a prediction is that insertion of $c c 2$ close to a region where neocentromeres can form will only result in CENP-A ${ }^{\text {Cnp1 }}$ incorporation when an active neocentromere is present. We therefore inserted cc2 at locations $73 \mathrm{~kb}$ (itg6), $60 \mathrm{~kb}$ (itg7) and $7 \mathrm{~kb}$ (itg8) from the neo1R region in cells with a wildtype cen 1 (no sub-telomeric neocentromere) or with an active neocentromere neo1R (wild-type cen1 deleted) (Figure 4A). 
Unlike wild-type cells where itg8 was spatially distant from the SPB, itg8 was positioned close to the SPB-centromere cluster in 96\% of neo1R cells (Figure 4C-E). CENP-A ${ }^{\text {Cnp1 }}$ was enriched on lys 1:cc2, but not itg8:cc2, in cells with wild-type cen1 (Figure 2D; Figure 4B). However, in cells with cen1د

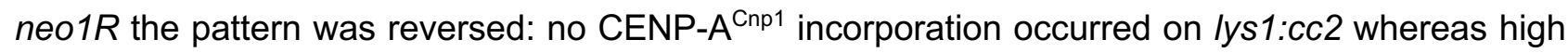
levels of CENP-A ${ }^{\mathrm{Cnp} 1}$ were detected on itg8:cc2 located $8 \mathrm{~kb}$ from the active neo1 $R$ neocentromere. Little or no CENP-A ${ }^{\text {Cnp1 }}$ was detected on the itg6:cc2 and itg7:cc2 insertions at greater distances from this neocentromere (Figure 4B). In cells with CENP-A ${ }^{C n 1}$ incorporation at itg8:cc2 we tested for CENP-A $\mathrm{A}^{\mathrm{Cnp} 1}$ enrichment at five positions (sites $\mathrm{i}-\mathrm{v}$ ) between itg8:cc2 and the active neo1R centromere (Figure 4A). As expected, high levels of CENP-A ${ }^{\text {Cnp1 }}$ were detected at two positions (sites vi, vii) within the characterized neo1R neocentromere (Ishii et al., 2008). However, substantial CENP-A ${ }^{\text {Cnp1 }}$ incorporation was only observed $5.2 \mathrm{~kb}$ from neo1R (site i; $1.6 \mathrm{~kb}$ from itg8) when $c c 2$ had been inserted at itg8, whereas little or no CENP-A ${ }^{\mathrm{Cnp} 1}$ enrichment was detected at sites i-v between itg 8 and neo1R (Figure 4F).

These analyses demonstrate that deletion of native cen1 prevents de novo CENP-A ${ }^{\text {Cnp1 }}$ incorporation on $c c 2$ subsequently inserted at lys 1 but permits CENP-A ${ }^{\text {Cnp1 }}$ assembly on $c c 2$ when inserted close to a resulting neocentromere. The fact that CENP-A ${ }^{\mathrm{Cnp} 1}$ is not detected at most positions between the neo1R centromere and itg8:cc2 indicates that, as at native cen1 (Figure S4D), CENP-A ${ }^{\text {Cnp1 }}$ chromatin does not spread uniformly from the pre-existing neocentromere to the nearby inserted cc2 DNA. We conclude that it is the proximity of lys 1 or itg 8 to functional centromeres, rather than properties of sequences immediately flanking these loci that allows the naturally CENP-A $A^{\text {Cnp1. }}$ permissive $c c 2$ DNA substrate to assemble CENP-A $A^{C n p 1}$ when inserted these locations.

\section{Centromeric heterochromatin is not required for de novo CENP-A ${ }^{\mathrm{Cnp} 1}$ incorporation on} centromere DNA placed close to an existing centromere

In minichromosome-based establishment assays $\mathrm{H} 3 \mathrm{~K} 9$ me-dependent heterochromatin is needed to

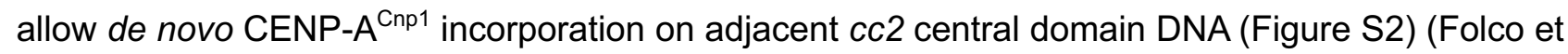
al., 2008). If the nuclear environment formed by SPB-centromere clustering is sufficient to promote de novo CENP-A ${ }^{\text {Cnp1 }}$ assembly, a prediction is that centromeric heterochromatin would not be required when central domain DNA is inserted close to endogenous centromeres. The lys1:cc2 insertion is positioned only $11.3 \mathrm{~kb}$ from endogenous cen 1 heterochromatic dh/otr1 repeats (Figure 
S5A). To determine if centromeric heterochromatin influences CENP-A ${ }^{\text {Cnp1 }}$ chromatin establishment at lys 1 we inserted cc2 DNA at this locus in either wild-type (wt) or heterochromatin-deficient $c / r 4 \Delta$ cells (lack Clr4 H3K9 methyltransferase). FISH confirmed that the lys1 locus and lys1:cc2 insertion remain near SPBs in cells lacking Clr4 (Figure S5B-E). qChIP demonstrated that CENP-A ${ }^{\text {Cnp1 }}$ was established on lys1:cc2 insertions made in either wild-type or clr4 $\Delta$ cells and that both CENP-C ${ }^{\text {Cnp3 }}$ and $\mathrm{Knl} 1^{\mathrm{Spc7}}$ kinetochore proteins were recruited (Figure S5F-H). Thus, the de novo assembly of CENP-A ${ }^{\text {Cnp1 }}$ and kinetochore proteins at lys1:cc2 occurs independently of nearby centromeric heterochromatin.

We conclude that centromeric heterochromatin is not required to assemble CENP-A $\mathrm{A}^{\mathrm{Cnp} 1}$ and kinetochore proteins on freshly introduced centromeric DNA if that DNA is positioned in cis close to an existing centromere which clusters with other centromeres, and associated CENP-A ${ }^{\text {Cnp1 }}$ plus its assembly factors, around SPBs. The placement of centromeric central domain DNA close to active centromeres bypasses the requirement for heterochromatin. This lack of a need for centromeric heterochromatin is consistent with heterochromatin normally influencing establishment of CENP$A^{\text {Cnp1 }}$ chromatin by sequestering freshly introduced centromeric DNA at SPBs.

\section{Direct tethering of centromeric DNA to SPBs mediates establishment of CENP-A ${ }^{\text {Cnp1 }}$ chromatin}

Insertion of central domain cc2 DNA near endogenous centromeres indicates that proximity in cis to SPB-centromere clusters enhances CENP-A ${ }^{\mathrm{Cnp} 1}$ chromatin establishment. If the SPB-centromere cluster creates a nuclear compartment that promotes CENP-A ${ }^{\mathrm{Cnp} 1}$ assembly then positioning centromeric cc2 DNA in trans near SPBs might also lead to CENP-A ${ }^{\mathrm{Cnp} 1}$ and kinetochore assembly. To directly test if the SPB-centromere compartment influences CENP-A ${ }^{\text {Cnp1 }}$ chromatin establishment on centromeric DNA we artificially tethered episomal minichromosomes to SPBs. The inner nuclear membrane (INM) protein Lem2 localizes around the nuclear envelope and also exhibits strong colocalization with SPBs (Figure 5A) (Ebrahimi et al., 2018). Lem2 is also specifically enriched across the central domain of fission yeast centromeres (Barrales et al., 2016; Iglesias et al., 2020). Arrays of lacO sites (2.8 kb; $~ 90$ lacO sites) were placed in pcc2, generating pcc2-lacO (Table S2) which was then transformed into cells constitutively expressing a Lacl-GFP fusion protein (binds pcc2-lacO) and Lem2 fused to both GFP-binding protein (GBP) and mCherry (Lem2-GBP-mCherry; 
Figure 5A, B). Therefore, cells expressing both Lem2-GBP-mCherry and Lacl-GFP should tether pcc2-lacO to SPBs. Indeed, Lem2-mediated tethering resulted in the pcc2-lacO FISH signal being in close proximity to SPBs in $77 \%$ of cells, whereas in the absence of tethering components it was located away from SPBs in $>77 \%$ of cells examined (Figure 5C, D). Crucially, this Lem2-mediated

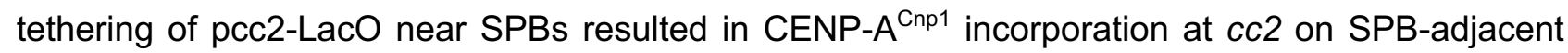
pcc2-lacO, whereas CENP-A ${ }^{\text {Cnp1 }}$ was not detected on untethered pcc2-lacO or pcc2 itself (Figure $5 E)$. In addition to CENP-A $A^{C n p 1}$, the inner kinetochore protein CENP- $C^{C n p 3}$ and outer kinetochore protein $\mathrm{Knl} 1^{\mathrm{Spc} 7}$ were also assembled on the $c c 2$ central domain of pcc2-lacO, but only when it was tethered at SPBs (Figure 5F, G).

These analyses demonstrate that direct tethering of $c c 2$ DNA to SPBs enables CENP-A ${ }^{\text {Cnp1 }}$ chromatin to be established without the need for adjacent heterochromatin. However, Lem2 is not an SPB-specific protein and thus Lem2-mediated pcc2-lacO tethering does not rule out the possibility that the non-SPB fraction of Lem2, localized around the nuclear envelope (Figure 5A), somehow contributes to CENP-A ${ }^{\text {Cnp1 }}$ and kinetochore protein enrichment. The Alp4 and Alp6 proteins are components of the SPB associated $\mathrm{y}$-tubulin complex and a proportion of both proteins localize on the nucleoplasmic side of SPBs in interphase (Bestul et al., 2017). Cells expressing Alp4-GBPmCherry or Alp6-GBP-mCherry fusion proteins and Lacl-GFP, transformed with pcc2-LacO were therefore generated (Figure S6A, B). Both Alp4-GBP- or Alp6-GBP-mediated tethering resulted in pcc2-lacO being located close to SPBs in $82-90 \%$ of cells, whereas in $>79 \%$ of cells lacking tethering components pcc2-lacO was located distant from SPBs (Figure S6C-E). Importantly, SPB tethering

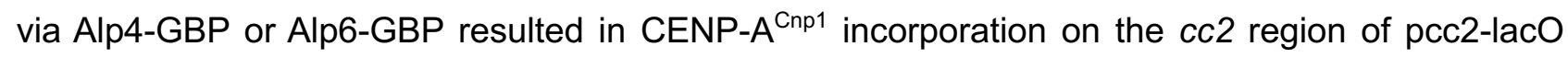
(Figure S6F, G). Thus, the direct tethering of cc2 DNA to SPBs via SPB-specific components enables CENP-A ${ }^{\text {Cnp1 }}$ chromatin establishment. The establishment of CENP-A ${ }^{\text {Cnp1 }}$ chromatin on pcc2-lacO transformed into Lem2-GBP/Lacl-GFP-expressing cells was unaffected by the absence of Clr4 H3K9 methyltransferase (clr4s; Figure S7).

Together these manipulations reveal that in the absence of adjacent heterochromatin the forced localization of centromeric central domain DNA, the native substrate for fission yeast CENP-A ${ }^{\text {Cnp1 }}$ assembly, to SPBs is sufficient to trigger CENP-A ${ }^{\text {Cnp1 }}$ chromatin and kinetochore assembly. 


\section{Loss of centromere-SPB association prevents CENP-A ${ }^{\mathrm{Cnp} 1}$ chromatin establishment}

If the de novo establishment of CENP-A $\mathrm{A}^{\mathrm{Cnp} 1}$ chromatin on centromeric DNA tethered near SPBs depends on the surrounding nuclear compartment, then loss of centromere-SPB association would be expected to hinder CENP-A ${ }^{\text {Cnp1 }}$ incorporation. The accumulation of Lem2 at SPBs requires the Csi1 protein; in cells lacking Csi1 (csi1 $\Delta$ ) Lem2 is mainly localized around the nuclear periphery (Figure 6A) (Ebrahimi et al., 2018). We therefore used csi1s cells to test if loss of the SPBassociated Lem2 pool affects Lem2-mediated tethering of pcc2-lacO at SPBs (Figure 6A, B). Indeed, pcc2-lacO was located near SPBs in only $23 \%$ of csi1 1 cells compared to $77 \%$ of wild-type cells expressing Lem2-GBP-mCherry and Lacl-GFP (Figure 6D, E). Furthermore, csi1 $\Delta$ cells were unable to establish CENP-A ${ }^{\text {Cnp1 }}$ chromatin on Lem2-tethered pcc2-lacO (Figure 6F). However, CENP-A ${ }^{\text {Cnp1 }}$ can assemble de novo on cc2 of pHcc2 transformed into csi1 $\Delta$ cells (Figure 6G), indicating that Csi1 itself is not required for CENP-A ${ }^{\text {Cnp1 }}$ establishment. Thus, Lem2 needs to be concentrated at SPBs

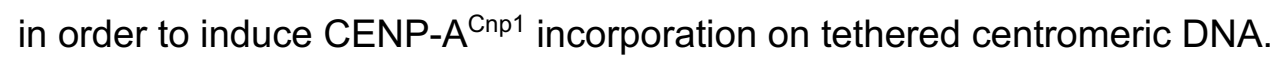

Together these data indicate that pericentromeric heterochromatin is sufficient to mediate frequent contact with SPBs where centromeres and CENP-A ${ }^{\mathrm{Cnp} 1}$ assembly factors are concentrated. We conclude that heterochromatin promotes exposure of adjacent $c c 2$ centromere DNA to this CENP. $A^{\text {Cnp1 }}$ assembly factor-rich nuclear sub-compartment, thus ensuring the assembly of CENP-A chromatin and kinetochores.

\section{Discussion}

Assembly of CENP-A chromatin is epigenetically regulated. Here we demonstrate that, in addition to the impact of chromatin context and prior CENP-A history, spatial location within the nucleus is an epigenetic influence on the chromatin fate of centromeric DNA. We show that heterochromatin causes minichromosomes to localize near SPBs, providing a likely explanation for the role of heterochromatin in promoting CENP-A ${ }^{\mathrm{Cnp} 1}$ chromatin establishment on adjacent centromeric sequences. By placing a CENP-A ${ }^{\mathrm{Cnp} 1}$ assembly-competent sequence $(c c 2)$ in various spatial contexts we demonstrate that being in the vicinity of centromere clusters at SPBs triggers CENP. $\mathrm{A}^{\text {Cnp1 }}$ chromatin establishment. 
Despite epigenetic factors being important in the establishment of CENP-A chromatin, certain sequences are preferred, including human $\alpha$-satellite arrays and fission yeast central domains. Rather than the precise sequence being critical, evidence suggests that innate properties of central domain regions, such as their unusual transcriptional landscape and high rates of histone $\mathrm{H} 3$

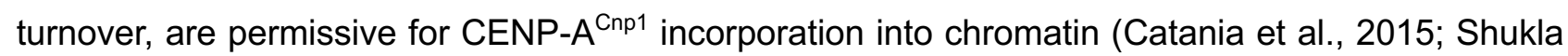
et al., 2018; Singh et al., 2020). Although central domain sequences are the preferred substrate for CENP-A ${ }^{\text {Cnp1 }}$ assembly in fission yeast, de novo assembly of CENP-A ${ }^{\text {Cnp1 }}$ chromatin is context dependent. Outer repeat-directed or synthetic heterochromatin promotes CENP-A ${ }^{\text {Cnp1 }}$ chromatin establishment on adjacent central domain DNA (Folco et al., 2008; Kagansky et al., 2009). CENP$A^{\text {Cnp1 }}$ overexpression induces de novo CENP-A ${ }^{\text {Cnp1 }}$ chromatin establishment on plasmid-based minichromosomes devoid of heterochromatin and carrying only central domain sequences (Catania et al., 2015).

We have previously suggested two models to explain these observations (Catania et al., 2015; Choi et al., 2012; Folco et al., 2008). In the 'modifier' model, heterochromatin performs a chromatindirected role such as recruitment of histone-modifying enzymes or remodelers that influence histone

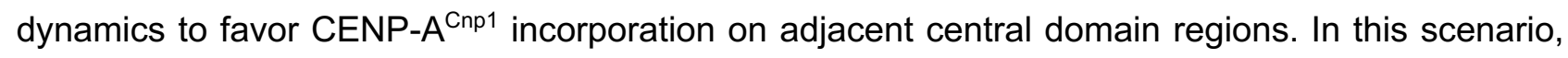
CENP-A ${ }^{\text {Cnp1 }}$ overexpression would shift the equilibrium away from transcription-dependent histone $\mathrm{H} 3$ recycling and towards CENP-A $\mathrm{A}^{\mathrm{Cnp} 1}$ deposition. In the 'positioning' model, the role of heterochromatin, due to its own localization, would place central domain DNA at a nuclear location permissive for CENP-A ${ }^{\text {Cnp1 }}$ deposition, such as a compartment exhibiting high levels of CENP-A ${ }^{\text {Cnp1 }}$ and associated assembly factors. In this model, overexpression of CENP-A ${ }^{\text {Cnp } 1}$ would bypass heterochromatin's function by making a greater proportion of nuclear space permissive for CENP. $A^{\text {Cnp1 }}$ assembly.

Here we have utilized FISH to demonstrate that minichromosome-borne heterochromatin preferentially locates close to SPBs. We hypothesize that any sequence positioned at this location will be exposed to high concentrations of CENP-A ${ }^{\text {Cnp1 }}$ and its assembly factors because centromeres are clustered at SPBs for most of the cell cycle. However, only sequences such as centromeric central domain DNA, with embedded properties that drive transcription-coupled $\mathrm{H} 3$ replacement with CENP-A ${ }^{\text {Cnp1 }}$, actually incorporate CENP-A ${ }^{\text {Cnp1 }}$ (Shukla et al., 2018). 
Support for the hypothesis that heterochromatin's role in CENP-A ${ }^{\text {Cnp1 }}$ establishment is to position central domain within the SPB-centromere cluster compartment of the nucleus is provided by our finding that centromeric cc2 DNA inserted close to endogenous or neo- centromeres assembled CENP-A ${ }^{\text {Cnp1 }}$ chromatin, whereas $c c 2$ inserted at locations far away from centromeres did not. The positioning of lys1 and itg 8 close to SPBs in wild-type and neocentromere-containing cells, respectively, correlates with the incorporation of CENP-A $A^{C n p 1}$ on $c c 2$ when inserted at these sites. Although the failure of CENP-A ${ }^{\text {Cnp1 }}$ to assemble on centromere-distal sites such as ade 3 could be attributed to selection against deleterious dicentric formation on this endogenous chromosome, we have previously shown that $c c 2$ present on the arm of a 530-kb non-essential linear minichromosome also does not normally assemble CENP-A ${ }^{\text {Cnp1 }}$. However, that minichromosome is capable of dicentric formation because overexpressed CENP-A $\mathrm{A}^{\mathrm{Cnp} 1}$ incorporates into $c c 2$ and causes missegregation (Catania et al., 2015). Thus, placing central domain DNA near centromeres in cis results in CENP$\mathrm{A}^{\mathrm{Cnp1}}$ incorporation. Moreover, direct tethering of minichromosome-borne central domain DNA in trans to SPB-associated proteins also triggered de novo assembly of CENP-A ${ }^{\text {Cnp1 }}$ chromatin, bypassing the requirement for heterochromatin. Thus, when susceptible sequences are positioned in the vicinity of SPBs, establishment of CENP-A ${ }^{C n p 1}$ chromatin is uncoupled from the presence of heterochromatin. These observations indicate that nuclear positioning is an epigenetic factor important for establishing centromere function and the function that heterochromatin provides is positioning information.

Fission yeast neocentromeres arise most frequently in subtelomeric regions and immature neocentromeres near rDNA can be stabilized by relocation to subtelomeric regions or upon acquisition of adjacent heterochromatin (Ishii et al., 2008; Ogiyama et al., 2013). When

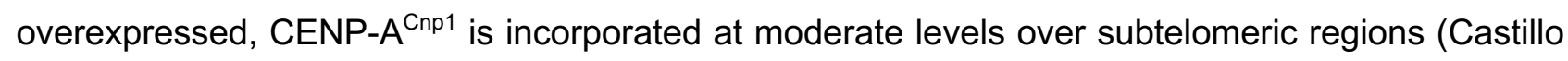
et al., 2013). Therefore, subtelomeric regions represent favored, but secondary, sites for CENP-A ${ }^{\text {Cnp1 }}$ and kinetochore assembly. H3K9me-dependent heterochromatin is normally assembled adjacent to telomeres (Kanoh et al., 2005). During interphase fission yeast telomeres are attached to the nuclear envelope via inner nuclear membrane proteins Bqt3 and Bqt4 (Chikashige et al., 2009). Moreover, $\mathrm{Hi}-\mathrm{C}$ analysis detects frequent contacts between telomere and centromere regions (Mizuguchi et al., 2014). We suggest that as consequence of their association with the nuclear periphery, subtelomeric regions perform a highly constrained exploration of the nucleus, making them more likely to meet 
the SPB-centromere cluster which exposes them to the immediate nuclear compartment containing high levels of CENP-A ${ }^{\text {Cnp1 }}$ assembly factors. Thus, association with the nuclear envelope offers an attractive explanation for the subtelomeric location of most fission yeast neocentromeres. Neocentromeres arise at telomeres even in the absence telomeric heterochromatin (Ishii et al., 2008), suggesting that it is telomere anchoring at the nuclear envelope, not subtelomeric heterochromatin, that designates them as secondary CENP-A ${ }^{\text {Cnp1 }}$ assembly sites.

In fission yeast, centromeres are clustered at the SPB throughout the cell cycle, apart from during mitosis, returning to the SPB in anaphase. CENP-A ${ }^{C n p 1}$ and several CENP-A ${ }^{\text {Cnp1 }}$ assembly factors and chaperones such as Scm3 ${ }^{\text {HJURP }}$, Mis16 ${ }^{\mathrm{RbAP} 46 / 48}$, Mis18, Eic1/Mis19 are concentrated on centromeres around the SPB during interphase (Hayashi et al., 2004; Pidoux et al., 2009; Subramanian et al., 2014; Williams et al., 2009). Mammalian centromeres are not localized close to centrosomes (SPB equivalent) during most of the cell cycle. However, after mitotic chromosome segregation, mammalian centromeres transiently cluster at spindle poles in late anaphase/telophase, subsequently dispersing during G1 (Gerlich et al., 2003). Centromere clustering is also pronounced in plants that exhibit an overt 'Rabl' configuration where centromeres and telomeres are clustered at opposite sides of interphase nuclei (Oko et al., 2020). Intriguingly, the Mis18 CENP-A assembly complex is normally recruited to human centromeres in late anaphase/telophase prior to arrival of the HJURP CENP-A chaperone and new CENP-A incorporation in early G1 (Zasadzińska and Foltz, 2017), although loss of CDK (cyclin-dependent kinase) regulation allows premature CENP-A deposition in G2 cells (Stankovic et al., 2017). Therefore, the centromeres of complex eukaryotes are briefly clustered together at precisely the time when assembly factors are recruited to centromeres. This spatio-temporal co-ordination may maximize the local concentration of CENP-A and its assembly factors to ensure the efficient removal of H3 placeholder nucleosomes and replenishment of CENP-A nucleosomes in centromeric chromatin (Dunleavy et al., 2011).

Once CENP-A $\mathrm{A}^{\mathrm{Cnp} 1}$ chromatin and kinetochores are assembled at fission yeast centromeres, it is clear that heterochromatin-independent connections with SPBs are established. Centromeres remain clustered at SPBs in the absence of pericentromeric H3K9me-dependent heterochromatin but SPBcentromere clustering is disrupted when essential kinetochore components such as Mis6 or Nuf2 are defective (Appelgren et al., 2003; Saitoh et al., 1997). Thus, once assembled, an intact 
bioRxiv preprint doi: https://doi.org/10.1101/2021.12.16.473016; this version posted December 16, 2021. The copyright holder for this preprint (which was not certified by peer review) is the author/funder, who has granted bioRxiv a license to display the preprint in perpetuity. It is made available under aCC-BY-NC-ND 4.0 International license.

interphase kinetochore structure rather than pericentromeric heterochromatin appears to provide the main physical link between functional centromeres and SPBs.

Here we have demonstrated that the specific location of centromere sequences within nuclei (i.e. their spatial context) exerts an epigenetic influence on the eventual CENP-A chromatin state attained by specific DNA sequences. Our analyses demonstrate that the SPB-centromere cluster forms a sub-compartment within the nucleus that promotes CENP-A and kinetochore assembly on DNA sequences presenting the required features to facilitate CENP-A chromatin assembly in place of canonical H3 chromatin. Thus, spatial positioning in the nucleus is a hitherto unrecognized epigenetic determinant of centromere identity.

\section{Author Contributions}

Conceptualization, W.W., A.L.P. and R.C.A.; Methodology, W.W., T.M., D.A. and A.L.P.; Investigation, W.W.; Visualization, W.W.; Writing - First Draft, W.W.; Writing - Review \& Editing, W.W., A.L.P. and R.C.A.; Funding Acquisition, R.C.A.; Supervision, A.L.P. and R.C.A.

\section{Acknowledgments}

We are grateful to members of the Allshire lab especially Manu Shukla, Nitobe London and Dominik Hoelper for helpful suggestions and comments on the manuscript and Sharon White for organizational support. Takeshi Urano is thanked for the 5.1.1 (H3K9me) antibody; Ken Sawin for the Cdc11 antibody; Kevin Hardwick for the Spc7 antibody; Julie Cooper for the plasmid pF6a-GBPmCherry-Hyg; and Nick Rhind for S. octosporus strain. W.W. is supported by the Darwin Trust of Edinburgh. R.C.A. is a Wellcome Principal Research Fellow (095021; 200885); the Wellcome Centre for Cell Biology is supported by core funding from Wellcome (203149). The Allshire lab dedicates this study to the memory of our dear colleague and friend Sasha Kagansky whose research and insights were an inspiration for this project.

\section{Declaration of Interests}

The authors declare no competing interests. 
bioRxiv preprint doi: https://doi.org/10.1101/2021.12.16.473016; this version posted December 16, 2021. The copyright holder for this preprint (which was not certified by peer review) is the author/funder, who has granted bioRxiv a license to display the preprint in perpetuity. It is made available under aCC-BY-NC-ND 4.0 International license.

\section{Figure Legends}

Figure 1. Centromeric heterochromatin associates with the SPB-centromere cluster

(A) Diagram of pHet, pcc2 and pHcc2 minichromosomes. Black bars above each plasmid map represent qChIP primer sites on ampicillin gene (amp), cc2 and $K$ " repeats of plasmids, respectively. Dashed red line on plasmids indicates position of FISH probe.

(B-D) qChIP analyses for H3K9me2 levels on amp gene of pHet (B), K" repeats of pHcc2 (D), dg

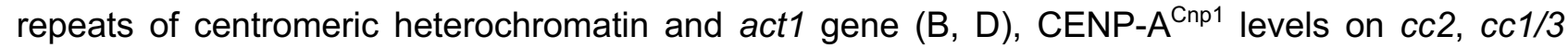
(indicates sequences common to $c c 1$ and $c c 3$ ) and act1 in wt and clr4 $\Delta$ cells containing $c c 2 \Delta:: c c 1$ at cen2 transformed with pHet (B), pcc2 (C) or pHcc2 (C, D). \%IP levels in S. pombe were normalized to \%IP of cen3 heterochromatin (HC) repeats from spiked-in S. octosporus chromatin in (B). qChIP results in (C, D) are reported as \%IP. Data are mean \pm SD (error bars) ( $n=3-4$ experimental replicates). ${ }^{*}, p<0.05 ;{ }^{* *}, p<0.005 ;{ }^{* * *}, p<0.0005$ (Unpaired t-test).

(E) Representative images of plasmid DNA FISH (red; probe as indicated in A), SPB location (green; anti-Cdc11) and DNA staining (blue, DAPI) in wt and clr4 $\Delta$ cells transformed with pcc2, pHcc2 or pHet. Images were scaled relative to the maximum values of histogram. Scale bar, $5 \mu \mathrm{m}$.

(F) Cells were classified into three groups according to the 3D distances between plasmid and SPB (Cdc11): overlap $(\leq 0.3 \mu \mathrm{m})$, adjacent $(0.3-0.5 \mu \mathrm{m})$ or separate $(0.5-3 \mu \mathrm{m})$. Percentage of interphase cells ( $n$, number analyzed from 3 independent experiments) in each category. Avg, average distance. ns, no significance; ${ }^{* *}, p<0.001 ;{ }^{* * *}, p<0.0001$ (Mann-Whitney $U$ test) (See also STAR Methods and Figures S1-S3). 
bioRxiv preprint doi: https://doi.org/10.1101/2021.12.16.473016; this version posted December 16, 2021. The copyright holder for this preprint (which was not certified by peer review) is the author/funder, who has granted bioRxiv a license to display the preprint in perpetuity. It is made available under aCC-BY-NC-ND 4.0 International license.

Figure 2. CENP-A ${ }^{\text {Cnp1 }}$ chromatin is established on centromere-adjacent lys1:cc2 central domain

(A) Ectopic cc2, carrying $880 \mathrm{bp} i m r 2 L, 6.8 \mathrm{~kb} c c 2$ (subdivided into K-to-Q regions; $6 \mathrm{~kb}$ is unique) and 920 bp imr2R DNA, were inserted at lys1 (lys1:cc2; $26 \mathrm{~kb}$ from cc1) or ade3 (ade3:cc2; $2438 \mathrm{~kb}$ from cc1) on Chrl in $c c 2 \Delta:: c c 1$ strain.

(B) Representative images of live cells expressing Sad1-dsRed (SPB marker) and Lacl-GFP bound to lys 1:lacO or ade3:lacO (Ding and Hiraoka, 2017). Images were scaled relative to the maximum intensity in the set of images. Scale bar, $5 \mu \mathrm{m}$.

(C) 3D distances between lys1:lacO or ade3:lacO and SPBs (Sad1). Percentage of G2 cells (n, number analyzed from 3 independent experiments) in each category, classified as in Figure 1. Avg, average distance. ${ }^{* *}, \mathrm{p}<0.0001$ (Mann-Whitney U test) (See also STAR Methods.)

(D) qChIP for CENP-A ${ }^{\text {Cnp1 }}$ at regions L-P of $c c 2, c c 1 / 3$ and act1 in wt cens strain carrying endogenous cen2-cc2 or cen2-cc2 $\Delta:: c c 1$ strain with lys1:cc2 or ade3:cc2 insertions. \# number indicates individual isolates.

$(E-G)$ qChIP analyses for CENP-C ${ }^{\mathrm{Cnp} 3}(\mathrm{E}), \mathrm{CENP}_{-} \mathrm{K}^{\mathrm{Sim} 4}(\mathrm{~F}), \mathrm{Knl}^{\mathrm{Spc7}}(\mathrm{G})$ levels at $c c 2, c c 1 / 3$ and act1 gene in in wt cens strain carrying endogenous cen2-cc2 or cen2-cc2 $:: c c 1$ strain with $/ y s 1: c c 2 . \% \mathrm{IP}$ levels in S. pombe were normalized to \%IP of S. octosporus central core from spiked-in chromatin in $(E)$. qChIP results in $(D, F, G)$ were reported as \%IP. Data are mean $\pm S D(n=3)$. ns, no significance; ${ }^{*}, p<0.05$ (Unpaired t-test) (See also Figure S1, S4, S5). 
bioRxiv preprint doi: https://doi.org/10.1101/2021.12.16.473016; this version posted December 16, 2021. The copyright holder for this preprint (which was not certified by peer review) is the author/funder, who has granted bioRxiv a license to display the preprint in perpetuity. It is made available under aCC-BY-NC-ND 4.0 International license.

Figure 3. neo1R neocentromere clusters with endogenous centromeres at the SPB during interphase

(A) Diagram represents strains with cen 1 or lacking cen 1 but carrying neo1R neocentromere (cen $1 \Delta$ $n e 01 R)$. Red line indicates position of neo1R DNA FISH probe (Chrl: 5,513,871-5,530,124).

(B, D) Representative images of neo1R DNAFISH (red; probe as indicated in A), SPB location (green; anti-Cdc11; B) or centromere clusters (green; anti-CENP ${ }^{\text {nnp1 }}$; D) and DNA staining (blue, DAPI) in wt cen1 $(\mathrm{B})$ and cen1 $\Delta$ neo1R cells. Images were scaled as in Figure 1. Scale bar, $5 \mu \mathrm{m}$.

(C, E) 3D distances between neo1R DNA and SPBs (Cdc11; C) or centromere clusters (CENP-A ${ }^{\text {Cnp1; }}$ E). Percentage of interphase cells ( $n$, number analyzed) in each distance category, classified as in Figure 1. Avg, average distance. ${ }^{* * *}, p<0.0001$ (Mann-Whitney $U$ test).

Figure 4. CENP-A ${ }^{\text {Cnp1 }}$ chromatin can establish on the inserted neocentromere proximalcentral domain

(A) Ectopic cc2 inserted at lys1, itg6 (Chrl: 5,435,010-5,435,237), itg7 (Chrl: 5,447,816-5,448,235) and itg 8 (Chrl: 5,501,647-5,502,134), $1.8 \mathrm{Mb}, 73 \mathrm{~kb}, 60 \mathrm{~kb}$ and $7 \mathrm{~kb}$ from neo1R CENP-A ${ }^{\text {Cnp1 }}$ domain

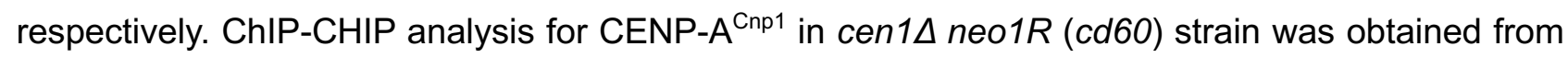
(Ishii et al., 2008). Red lines indicate itg8 and 7 qChIP primer sites (i-vii).

(B) qChIP analyses of CENP-A ${ }^{\text {Cnp } 1}$ levels at $c c 2, c c 1 / 3$ and act1 in wt cen 1 or cen $1 \Delta$ neo1R strain with lys1:cc2, itg6:cc2, itg7:cc2 or itg8:cc2 insertions (genome positions as indicated in A).

(C) Diagram represents wt-cen1 or cen1 1 neo1R strains. Red line indicates position of itg8 DNA FISH probe (Chrl 5,495,975-5,508,459).

(D) Representative images of itg8 DNA FISH (red; probe as indicated in C), SPB location (green; anti-Cdc11; B) and DNA staining (blue, DAPI) in wt cen1 (B) and cen1 $\Delta$ neo1R cells. Images scaled as in Figure 1. Scale bar, $5 \mu \mathrm{m}$.

(E) 3D distances between itg8 and SPBs (Cdc11), percentage of interphase cells ( $n$, number analyzed) in each category, classified as in Figure 1. Avg, average distance. ${ }^{* * *}, p<0.0001$ (MannWhitney $U$ test).

(F) qChIP analyses for CENP-A ${ }^{\text {Cnp1 }}$ levels at 7 loci (i-vii, positions as indicated in A) and act1 in cen1 $\Delta$ neo1R strain with or without itg8:cc2 insertion. \%IP levels in S. pombe were normalized to \%IP of $S$. octosporus central core from spiked-in chromatin $(B, F)$. Data are mean $\pm S D(n=3)$. ns, no significance; ${ }^{*}, p<0.05 ;{ }^{* *}, p<0.005 ;{ }^{* * *}, p<0.0005$ (Unpaired t-test). 


\section{Figure 5. Tethering $c c 2$ DNA to Lem2 allows CENP-A ${ }^{\text {Cnp1 }}$ incorporation and kinetochore protein recruitment}

(A) Representative images of live cells expressing Lem2-GFP and Sad1-dsRed or Lacl-GFP and Lem2-GBP-mCherry. Images were scaled as in Figure 2. Scale bar, $5 \mu \mathrm{m}$.

(B) Schematic representation of the tethering system used to force pcc2-lacO association with Lem2GBP-mCherry at the NE and SPB. pcc2-lacO is bound by Lacl-GFP and ultimately tethered to Lem2GBP-mCherry via GFP/GBP interaction.

(C) Representative images of cc2 DNA FISH (red), SPB location (green; anti-Cdc11) and DNA staining (blue, DAPI) in wt cens strain carrying endogenous cen2-cc2 or cen2-cc2 $\Delta:: c c 1$ strain expressing Lacl-GFP or both Lacl-GFP and Lem2-GBP-mCherry transformed with pcc2 or pcc2lacO. Fluorescence of Lacl-GFP and Lem2-GBP-mCherry was dissipated by the Immunofluorescence/DNA-FISH procedure and did not contribute punctate signal. Images were scaled as in Figure 1. Scale bar, $5 \mu \mathrm{m}$.

(D) 3D distances between $c c 2$ and SPBs (Cdc11), percentage of interphase cells ( $n$, number analyzed) in each category, classified as in Figure 1. Avg, average distance. ns, no significance; ${ }^{* * *}$, $p<0.0001$ (Mann-Whitney $U$ test).

$(E-G)$ qChIP analyses for CENP-A ${ }^{\mathrm{Cnp} 1}(\mathrm{E}), \mathrm{CENP}_{-} \mathrm{C}^{\mathrm{Cnp} 3}(\mathrm{~F}), \mathrm{Knl} 1^{\mathrm{Spc} 7}(\mathrm{G})$ levels at $c c 2, c c 1 / 3$ and act1 in wt cens strain carrying endogenous cen2-cc2 or cen2-cc2 $\Delta:: c c 1$ strain expressing Lacl-GFP or Lem2-GBP-mCherry or both of them transformed with pcc2 or pcc2-lacO. \%IP levels in S. pombe were normalized to \%IP of S. octosporus central core from spiked-in chromatin in (E, F). qChIP results in $(G)$ reported as \%IP. Data are mean $\pm S D(n=3) .{ }^{* *}, p<0.005 ;{ }^{* * *}, p<0.0005$ (Unpaired ttest) (See also Figure S6, S7). 
bioRxiv preprint doi: https://doi.org/10.1101/2021.12.16.473016; this version posted December 16, 2021. The copyright holder for this preprint (which was not certified by peer review) is the author/funder, who has granted bioRxiv a license to display the preprint in perpetuity. It is made available under aCC-BY-NC-ND 4.0 International license.

Figure 6. Loss of Csi1 prevents CENP-A ${ }^{\mathrm{Cnp} 1}$ chromatin establishment on Lem2-tethered pcc2IacO

(A, C) Representative images of live wt and csi1 $\Delta$ cells expressing Lem2-GFP and Sad1-dsRed (A) or Lacl-GFP and Lem2-GBP-mCherry (C). Images were scaled as in Figure 2. Scale bar, $5 \mu \mathrm{m}$.

(B) Forced association of pcc2-lacO with Lem2-GBP-mCherry at NE in csi1 $\Delta$ using same tethering system as in Figure 5. In csi1 1 , pcc2-lacO is expected to detach from the SPB due to loss of Lem2 from SPB.

(D) Representative images of cc2 DNA FISH (red), SPB location (green; anti-Cdc11) and DNA staining (blue, DAPI) wt or csi1 $\Delta$ strains expressing both Lacl-GFP and Lem2-GBP-mCherry transformed with pcc2 or pcc2-lacO. Images were scaled as in Figure 1. Scale bar, $5 \mu \mathrm{m}$.

(E) Percentage of interphase cells ( $n$, number analyzed) displaying distinct degrees of $c c 2$ DNA colocalization with SPBs (Cdc11). Cells were classified into three groups as in Figure 1. Avg, average distance. ns, no significance; ${ }^{* * *}, \mathrm{p}<0.0001$ (Mann-Whitney $U$ test).

$(F, G)$ qChIP analyses for CENP-A ${ }^{C n p 1}$ at $c c 2, c c 1 / 3$ and act1 in indicated strains transform with pcc2 or pcc2-lacO $(\mathrm{F})$ or pHcc2 $(\mathrm{G})$. qChIP primer site on pHcc2-borne $c c 2$ is indicated as black bar above plasmid map (G). \%IP levels in S. pombe were normalized to \%IP of S. octosporus central core from spiked-in chromatin. Data are mean $\pm S D(n=3)$. ns, no significance; * $p<0.05$ (Unpaired t-test). 
Figure 7. Model: Centromere identity is influenced by nuclear spatial organization

Due to clustering of endogenous centromeres (CENP-A ${ }^{\text {Cnp1 }}$-assembled central domains; red circles; heterochromatic outer repeats: green) at SPBs and incorporation of CENP-A ${ }^{\mathrm{Cnp} 1}$ at centromeres in G2, the zone around SPBs forms a nuclear sub-compartment rich in CENP-A ${ }^{\text {Cnp1 }}$ and its assembly factors (red shaded cloud). Ectopic central domain (outlined circles) inserted at centromere-proximal sites are exposed the high-CENP-A $\mathrm{A}^{\mathrm{Cnp} 1} \mathrm{SPB} / \mathrm{centromere}$ sub-compartment, promoting de novo incorporation of CENP-A ${ }^{\text {Cnp1 }}$, unlike centromere-distal locations. Similarly, only minichromosomes bearing heterochromatin, which mediates association with the SPB, exposes adjacent central domain to the high-CENP-A $A^{\text {Cnp1 }}$ SPB/centromere sub-compartment, resulting in CENP-A ${ }^{\text {Cnp1 }}$ incorporation. Heterochromatin, green; CENP-A ${ }^{\mathrm{Cnp} 1}$, red; neutral H3 chromatin, grey. 


\section{Methods}

\section{Yeast strains}

Yeast strains used in this study and their genotypes are listed in Table S1.

Standard genetic and molecular methods were used as described (Moreno et al., 1991). All ectopic $c c 2$ insertions were made in $c c 2 \Delta:: c c 1$ strains (Catania et al., 2015) by integrating linear cen2 central domain constructs ( $\sim 80 \mathrm{bp}$ imr2L, $-6.8 \mathrm{~kb} c c 2$ and $\sim 920 \mathrm{bp} i m r 2 R$, abbreviated as cc2) by homologous recombination (HR). pMC52 (Table S2), bearing $8.6 \mathrm{~kb}$ of $c c 2$ and kanMX6 selection cassette, was used as a starting plasmid for linear cc2 constructs. Two flanking DNA fragments of the desired target locus for cc2 insertions were amplified using primers listed in Table S3 by PCR. Restriction enzyme Kpnl/Xhol-digested first fragment was cloned into Kpnl/Xhol-digested pMC52, which were then digested by Sacl/Mscl and ligated with Sacl/Mscl-digested second PCR fragment by T4 DNA ligase (M0202S; NEB). Linear cc2 constructs were obtained by Sacl/Kpnl digestion of the resulting plasmids and transformed into desired strain for $c c 2$ insertion.

For the construction of Lem2/Alp4/Alp6-GBP-mCherry and Lem2-GFP, the GBP-mCherry-hygMX6 and GFP-natMX6 cassette in plasmid pFA6a-GBP-mCherry-hygMX6 (Fernández-Álvarez et al., 2016) and pFA6a-GFP-NatMX6 were amplified by PCR and integrated into genome by HR (Bähler et al., 1998).

clr4 $\Delta$ mutant was created by CRISPR/Cas9 method as described previously (Torres-Garcia et al., 2020). Briefly, clr4 gene specific sgRNA was cloned into Cas9 containing pLSB-KAN plasmid by Golden Gate Assembly kit (E1601S, NEB). The resulting plasmid clr4-pLSB-KAN and clr4 HR template obtained by annealing primer pair WW748-clr4-HR-F/WW749-clr4-HR-R (Table S3) were co-transformed into $S$. pombe by sorbitol-electroporation method.

Transformants were grown on appropriate selection plates and screened for correct integration or clr4 $\Delta$ mutant by yeast colony PCR using primers listed in Table S3. All plasmids and primers used in this study are listed in Table S2, Table S3 respectively.

\section{Yeast growth medium and conditions}


bioRxiv preprint doi: https://doi.org/10.1101/2021.12.16.473016; this version posted December 16, 2021. The copyright holder for this preprint (which was not certified by peer review) is the author/funder, who has granted bioRxiv a license to display the preprint in perpetuity. It is made available under aCC-BY-NC-ND 4.0 International license.

\section{Yeast growth medium and conditions}

All strains were grown at $32^{\circ} \mathrm{C}$ in YES (Yeast Extract with Supplements) rich medium or PMG (Pombe Minimal Glutamate) minimal medium, as appropriate. Selection for dominant markers was performed on YES medium supplemented with $100 \mu \mathrm{g} / \mathrm{ml}$ clonNAT (96736-11-7, Werner BioAgents), $100 \mu \mathrm{g} / \mathrm{ml}$ G418 (10131027, Gibco), or $123 \mu \mathrm{g} / \mathrm{ml}$ HygMX6 (31282-04-9, Duchefa Biochemie). clr4 $\Delta$ transformants were selected on YES supplemented with G418 plate and re-streaked to non-selective YES medium to allow loss of plasmid clr4-pLSB-KAN. Transformants with $c c 2$ insertions were selected on YES supplemented G418. Plasmids pcc2 (pMC2; carrying 8.6 kb of cc2) and pcc2-LacO (pMC12; carrying $8.6 \mathrm{~kb}$ of $c c 2$ and $2.8 \mathrm{~kb}$ of lacO) were selected on YES containing $100 \mu \mathrm{g} / \mathrm{ml} \mathrm{G418}$ in wt strains or on PMG-uracil in csi1 $($ csi14::ura4) strain. Strains carrying plasmid pHet (pMC183; carrying $2 \mathrm{~kb}$ of $K$ " repeats) or pHcc2 ( $\mathrm{H}$ denotes $5.6 \mathrm{~kb}$ of $K$ " repeats, $c c 2$ denotes $8.6 \mathrm{~kb}$ of $c c 2)$ were selected on YES supplemented with clonNAT or PMG-adenine-uracil medium respectively.

\section{Bacteria}

DH5a E. coli strains (C2987H, NEB) were grown in LB medium at $37^{\circ} \mathrm{C}$. E. coli competent cells carrying plasmids were selected on LB agar plates supplemented with $100 \mu \mathrm{g} / \mathrm{ml}$ of ampicillin or LB liquid supplemented with $50 \mu \mathrm{g} / \mathrm{ml}$ Carbenicillin (10177012; Invitrogen).

\section{Methods details}

\section{Yeast genetic crosses}

To obtain desired genotypes, two strains with opposite mating type (h+/h-) were mixed and grown on the nitrogen starved ME plate for sporulation at $32^{\circ} \mathrm{C}$ for 2 days. Asci were digested in glusulase (NEE-154, NEN) to release spores that were then plated on appropriate selective medium and grown at $32^{\circ} \mathrm{C}$.

\section{Yeast colony PCR}

Yeast strains were suspended in SPZ buffer (1.2 M sorbitol, $100 \mathrm{mM}$ sodium phosphate and 2.5 $\mathrm{mg} / \mathrm{ml}$ Zymolyase-100T (08320932, MP Biomedicals)) and incubated at $37^{\circ} \mathrm{C}$ for $30 \mathrm{~min}$. The resulted mixtures were used as PCR template for strain genotyping by Roche FastStart ${ }^{\mathrm{TM}}$ Taq polymerase PCR kit (12032953001, Roche) supplemented with primers. 


\section{Yeast transformation}

Yeast cells were transformed by sorbitol-electroporation method. Log phase cultures were harvested and resuspended in pre-transformation buffer (25 mM DTT, $0.6 \mathrm{M}$ sorbitol and $20 \mathrm{mM} \mathrm{HEPES,} \mathrm{pH7.6)}$ and incubated at $32^{\circ} \mathrm{C}$ with $180 \mathrm{rpm}$ shaking for $10 \mathrm{~min}$. Cells were washed three times in ice-cold 1.2 M sorbitol, mixed in an ice-cold cuvette with $200 \mathrm{ng}$ of plasmid DNA or purified DNA fragments obtained by QIAquick PCR Purification Kit (28104, QIAGEN) and then pulsed by an electroporator (Bio-Rad Gene Pulser II) at a setting of $2.25 \mathrm{kV}, 200 \Omega$ and $25 \mu \mathrm{F}$. Cells were either directly plated on medium with prototrophic selection directly or grown overnight in non-selective liquid before selection for antibiotic resistance (G418/cloNAT/HygMX6). Single colonies were isolated from selective medium.

\section{Minichromosome establishment assay}

Medium-sized colonies carrying circular plasmid-based minichromosome pHcc2 were replica-plated from PMG-adenine-uracil to PMG-low-adenine $(10 \mu \mathrm{g} / \mathrm{ml}$ adenine $)$ and incubated at $32^{\circ} \mathrm{C}$ for 2 days to determine functional centromere establishment frequency. Plasmid pHcc2 contains sup3e tRNA selection marker that suppresses ade6-704 mutation within strains, thus colony color on these PMGlow-adenine plates will indicate minichromosome loss (red colonies) or retention (white/pale pink colonies). In the absence of centromere establishment, minichromosomes behave as episomes that are rapidly lost. Minichromosomes that have established functional centromere segregate efficiently during mitosis. Minichromosomes occasionally integrate at genome will give a false-positive white phenotype. To assess the frequency of such integration events and to confirm establishment of centromere segregation function, colonies giving the white/pale-pink phenotype upon replica plating were re-streaked to single colonies on PMG-low-adenine plates. Red/white sectored colonies are indicative of centromere function with low levels of minichromosome loss, whereas pure white colonies are indicative of integration into endogenous chromosomes. Therefore, the percentage of sectored colonies number over the total colony numbers was used to represent centromere establishment frequency on minichromosome pHcc2.

\section{Quantitative chromatin immunoprecipitation (qChIP)}

Three independent cell cultures were grown in appropriate medium until log phase and fixed in $1 \%$ formaldehyde (F8775, MERCK) for 15 min followed by quenching in 125 mM Glycine (G8790, 
MERCK) at room temperature. ChIP was performed as previously described (Castillo et al., 2007). $2.5 \times 10^{8}$ cells were used for each ChIP. Briefly, cells were lysed by bead beating (Biospec) in $350 \mu l$ Lysis Buffer (50 mM Hepes-KOH pH 7.5, 140 mM NaCl, 1 mM EDTA, 1\% (v/v) Triton X-100 and 0.1\% (w/v) sodium deoxycholate) supplemented with $3.5 \mu$ l of 100 mM PMSF (329-98-6, MERCK) and 3.5 $\mu \mathrm{l}$ of $100 \mathrm{mM}$ yeast protease inhibitor (P8215, MERCK). Where indicated, $\sim 5 \times 10^{7}$ fixed, lysed S. octosporus cells (Rhind et al., 2011) were added to each initial crude cell lysates as a spike-in control. Crude cell lysates were sonicated using a Bioruptor (Diagenode) at $4^{\circ} \mathrm{C}$ on high voltage for $20 \mathrm{~min}$ (20 cycles of $30 \mathrm{~s}$ ON/OFF), followed by centrifugation at $13000 \mathrm{rpm}$ for $10 \mathrm{~min}$ to pellet cell debris. The resulting supernatant was used for following steps.

For H3K9me2 ChIP, $10 \mu$ lysate was retained as crude 'input' sample, whereas $300 \mu$ of the remaining lysates were incubated overnight with $20 \mu \mathrm{l}$ of washed protein G Dynabeads (10009D, Thermo Fisher Scientific) and $1 \mu \mathrm{l}$ of mouse anti-H3K9me2 (mAb5.1.1, gift from Takeshi Urano).

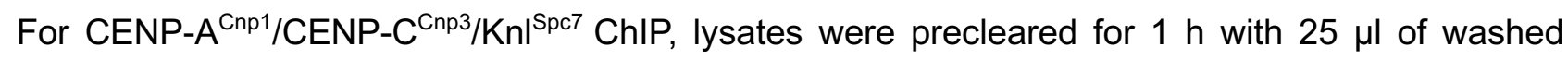
protein-G agarose beads (11243233001, Roche) and $10 \mu \mathrm{l}$ of precleared lysate was retained as crude 'input' sample. $300 \mu \mathrm{l}$ of the remaining pre-cleared lysates were incubated overnight with appropriate amount of antibody $\left(10 \mu \mathrm{l}\right.$ of sheep CENP-A ${ }^{\text {Cnp1 }}, C^{2}$ ENP-C ${ }^{\text {Cnp3 }}$, CENP-K ${ }^{\operatorname{Sim} 4}$ serum (Catania et al., 2015) (in-house preparation), $3 \mu$ of affinity-purified sheep anti-Spc7 (a gift from Kevin Hardwick) and $25 \mu$ of protein-G agarose beads.

After immunoprecipitation, the crude "IP" samples on beads were washed in Lysis Buffer, Lysis Buffer supplemented with $500 \mathrm{mM} \mathrm{NaCl}$, Wash Buffer (10 mM Tris-HCl pH 8, $250 \mathrm{mM} \mathrm{LiCl,} \mathrm{0.5 \%} \mathrm{IGEPAL}$ NP40 (56741, MERCK) 0.5\% (w/v) sodium deoxycholate and $1 \mathrm{mM}$ EDTA) and TE Buffer (10mM Tris- $\mathrm{HCl} \mathrm{pH} 8,1 \mathrm{mM}$ EDTA). DNA was recovered from input and IP samples using Chelex resin (1421253, BioRad). Quantitative PCR reactions (qPCR) were performed using a LightCycler 480 SybrGreen Master Mix (04887352001, Roche) and analyzed using Roche LightCycler software (version 1.5.1.62). Primers used for $\mathrm{qPCR}$ are listed in Table S3. ChIP enrichments on regions of interest were calculated as the ratio of "IP" sample to the corresponding "input" sample using the $\Delta \mathrm{CT}$ method and represented as \%IP. Where indicated, for spike-in qChIPs, \%IP levels in S. pombe were normalized to \%IP from spiked-in S. octosporus chromatin (specified in the figure legends). 


\section{Fluorescence microscopy}

Live fission yeast cells were mounted on a $2 \%$ agarose pad formed on $1 \mathrm{~mm}$ SuperFrost slides (Thermo Scientific) whereas fixed cells (immunofluorescence and DNA FISH) were mounted in VECTASHIELD Mounting Medium (H-1000-10, Vector Laboratories) on $1 \mathrm{~mm}$ Polysine slides (Thermo Scientific). Microscopy was performed with Nikon Ti2 inverted microscope equipped with a $\times 1001.49$ NA CFI Plan Apochromat TIRF objective, Lumencor Spectra X light source (Lumencor, Beaverton, OR USA) and a Photometrics Prime 95B camera (Teledyne Photometrics, Birmingham, UK), all controlled by Nikon NIS Elements software version 5.21.03 (RRID:SCR_014329). Filter sets from Semrock (Semrock, Rochester, New York, USA) were used to image Lem2-GFP, Lacl-GFP, Alexa Fluor 488 (A-11015, Invitrogen) at excitation 488 nm, emission 535 nm, Sad1-dsRed, Rhodamine at excitation $554 \mathrm{~nm}$, emission $590 \mathrm{~nm}$, Lem2/Alp4/Alp6-mCherry at excitation $578 \mathrm{~nm}$, emission $630 \mathrm{~nm}$ and DAPI, excitation $378 \mathrm{~nm}$, emission $460 \mathrm{~nm}$. A Mad City nano drive (Mad City Labs, Madison, WI, USA) was used to produce whole cell 3 dimensional (3D) images with a step size of $0.3 \mu \mathrm{m}$. All images were processed by Fiji software (RRID:SCR_002285). Live cell images were scaled relative to the maximum intensity in the set of images to allow comparison between images, but fixed cell images were scaled relative to the maximum value of histogram (specified in figure legends).

\section{Immunofluorescence/DNA FISH}

For Immunofluorescence/DNA FISH, cells were initially subjected to a similar Immunofluorescence protocol as described previously with some modifications (Castillo et al., 2007) and subsequent FISH process. Briefly, log phase yeast cultures were fixed with $3.7 \%$ formaldehyde for $7 \mathrm{~min}$ at room temperature, washed by PEM buffer (100 mM PIPES pH 7, $1 \mathrm{mM}$ EDTA, $1 \mathrm{mM} \mathrm{MgCl}$ ) and PEMS buffer (100 mM PIPES pH 7, $1 \mathrm{mM}$ EDTA, $1 \mathrm{mM} \mathrm{MgCl}$, $1.2 \mathrm{M}$ Sorbitol), followed by cell-wall digestion in PEMS buffer supplemented with $1 \mathrm{mg} / \mathrm{ml}$ Zymolyase-100T and $1 \mathrm{mg} / \mathrm{ml}$ Lallzyme (Lallzyme-MMX, Litmus Wines) at $37^{\circ} \mathrm{C}$ for $90 \mathrm{~min}$. After permeabilization in PEMS containing $1 \%$ Triton X-100 for 5 min at room temperature, cells were washed, blocked in PEMBAL (PEM containing 1\% BSA (A0281, MERCK), 0.1\% sodium azide, $100 \mathrm{mM}$ lysine hydrochloride (657-27-2, MERCK)) for $1 \mathrm{~h}$. Cells were then incubated overnight at $4{ }^{\circ} \mathrm{C}$ with 1:500 anti-Cdc11 (Castillo et al., 2007) (a

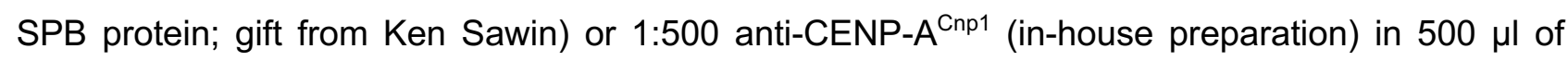
PEMBAL. Cells were then washed three times with PEMBAL and incubated overnight with 1:500 
Alexa-488-coupled donkey anti-sheep secondary antibody (A-11015, Invitrogen) in $500 \mu l$ of PEMBAL. Cells were then washed in PEMBAL and PEM buffer and re-fixed in $3.7 \%$ formaldehyde and $0.25 \%$ glutaraldehyde (111-30-8, MERCK) for $15 \mathrm{~min}$, washed with PEM buffer and treated with $1 \mathrm{mg} / \mathrm{ml}$ sodium borohydride in PEM buffer. After incubation with $2 \mu \mathrm{l}$ of $10 \mathrm{mg} / \mathrm{ml}$ RNase A (19101, Qiagen) in $100 \mu \mathrm{l}$ of PEMBAL at $37^{\circ} \mathrm{C}$ for $2 \mathrm{~h}$, cells were denatured in $100 \mu \mathrm{l}$ of freshly prepared 0.1 $\mathrm{M} \mathrm{NaOH}$ for $1 \mathrm{~min}$ and hybridized with $2 \mu$ of DNA FISH probe in $100 \mu$ l hybridization buffer (10\% Dextran sulphate (D8906, MERCK), 50\% deionized formamide (S4117, MERCK), 2XSSC, 5X Denhardts (D2532, MERCK), $0.5 \mathrm{mg} / \mathrm{ml}$ denatured salmon sperm DNA) at $37^{\circ} \mathrm{C}$ overnight.

For lys1, itg8 and neo1R FISH probe, a 12.5 kb region (Chrl: 3,727,604-3,737,389 and Chrl: 3,739,857-3,742,327) spanning lys1 gene, $\sim 12.5$ kb region (Chrl: 5,495,975-5,508,459) spanning itg8 locus (Chrl: 5,500,986-5,502,881) and $\sim 16.3 \mathrm{~kb}$ region (Chrl: 5,513,871-5,530,124) within neo1R CENP-A ${ }^{\text {Cnp1 }}$ domain were amplified by PCR using primers listed in Table S3 respectively. Plasmid pMC52, pMC1 was used to make cc2 and plasmid backbone DNAFISH probes, respectively. cc2 DNA FISH probe was used to locate $c c 2$ at endogenous cen2, lys1 and plasmid pcc2 and pHcc2, while plasmid backbone probe was used to locate pHet. FISH probes were obtained by DIG labeling 500 ng DNA (PCR products or plasmids) using DIG-Nick Translation Mix (11745816910, Roche) supplemented with $1 \mu$ l of 1:50 diluted DNase I (AM2222, Ambion).

After hybridization with DNA FISH probe, cells were washed with 2 XSSC containing $0.1 \%$ sodium azide and incubated with 1:100 sheep anti-DIG-Rhodamine (11207750910; Roche) in $100 \mu$ l of PBSBAG (PBS buffer supplemented with 1\% BSA (A0281, MERCK), 0.1\% sodium azide and $0.5 \%$ cold water fish gelatin (G7765, MERCK)) at room temperature overnight. Cells were finally stained with 4',6-diamidino-2-phenylindole (DAPI), mounted in VECTASHIELD Mounting Medium on Polylysine slides and imaged using Nikon NIS Elements software (version 5.21.03) on a Nikon Ti2 inverted microscope as indicated above. All images are scaled relative to the maximum value of histogram.

\section{D distance measurements}

3D distances between spots in two channels (green and red): Cdc11/CENP-A ${ }^{\text {Cnp1 }}$ (green) and DNA FISH (red) or lys1:lacO (ade3:lacO)/Lacl-GFP (green) and Sad1-dsred (red), were measured by Fiji using in-house script (https://doi.org/10.5281/zenodo.5657360). Briefly, the center of spot in each 
channel were determined in $\mathrm{X}-\mathrm{Y}$ using the Fiji "Find Maxima..." function with same threshold (Prominence $>500$ ), applied to a Z-projection. The Z-positions of each spot were then determined as the slice with the maximum pixel intensity at each $X-Y$ position. The distance to the nearest red spot for each green spot was reported if within $3 \mu \mathrm{m}$ representing the diameter of the fission yeast nucleus. If no red spot was detected with $3 \mu \mathrm{m}$ then that green spot was not included in the analysis. Distances between the resulting spots in each channel were measured by equation:

$$
d=\sqrt{ }\left(\Delta x^{2}+\Delta y^{2}+\Delta z^{2}\right)
$$

Live mono-nuclear cells 8-12 $\mu \mathrm{m}$ in length and only one SPB (Sad1-dsRed) nucleus-associated dot were recognized as G2 cells and subjected to distance measurements between Lacl-GFP (binds to lys1:lacO or ade3:IacO) and Sad1-dsRed. For immunofluorescence/DNA FISH, mononuclear cells with nuclear green-red spot pairs and only one SPB (Cdc11) or centromere cluster (CENP-A ${ }^{\text {Cnp1 }}$ ) spot were recognized as interphase cells and retained for distance measurement between DNA FISH locus (red) and protein Cdc11 or CENP-A ${ }^{\text {Cnp1 }}$ (green).

\section{Quantification and Statistical Analysis}

All quantification and statistical details of experiments are described in the figure legends or in the methods section. The qChIP results are obtained from more than 3 independent experimental replicates $(n \geq 3)$ and represented as mean \pm SD (standard deviation, error bars). Significance of the differences in qChIP results was evaluated using Unpaired t-test with a $p$ value threshold $<0.05$, by Prism Version 9.1.0 software (RRID:SCR_002798). 3D distance measurement results were obtained by analyzing $n$ number of interphase cells from 3 independent experimental replicates. Average distance for each strain were calculated and indicated as "Avg" (specified in figure legends). Cells were classified into three groups according to the distance: overlap $(\leq 0.3 \mu \mathrm{m})$, adjacent $(0.3-0.5 \mu \mathrm{m})$ or separate $(0.5-3 \mu \mathrm{m})$. The results were reported as percentage of cells (\% cells) in each group. For statistical significance analysis of distance data, Mann-Whitney $U$ test with a $p$ value threshold $<0.01$ was performed by Prism Version 9.1.0 software (RRID:SCR_002798) and the detailed results were showed in Table S4. 


\section{References}

Alfredsson-Timmins, J., Henningson, F., and Bjerling, P. (2007). The Clr4 methyltransferase determines the subnuclear localization of the mating-type region in fission yeast. J. Cell Sci. 120, 1935-1943.

Allshire, R.C., and Ekwall, K. (2015). Epigenetic Regulation of Chromatin States in Schizosaccharomyces pombe. Cold Spring Harb. Perspect. Biol. 7, a018770.

Allshire, R.C., and Karpen, G.H. (2008). Epigenetic regulation of centromeric chromatin: old dogs, new tricks? Nat. Rev. Genet. 9, 923-937.

Appelgren, H., Kniola, B., and Ekwall, K. (2003). Distinct centromere domain structures with separate functions demonstrated in live fission yeast cells. J. Cell Sci. 116, 4035-4042.

Bähler, J., Wu, J.Q., Longtine, M.S., Shah, N.G., McKenzie, A., 3rd, Steever, A.B., Wach, A., Philippsen, P., and Pringle, J.R. (1998). Heterologous modules for efficient and versatile PCR-based gene targeting in Schizosaccharomyces pombe. Yeast 14, 943-951.

Barrales, R.R., Forn, M., Georgescu, P.R., Sarkadi, Z., and Braun, S. (2016). Control of heterochromatin localization and silencing by the nuclear membrane protein Lem2. Genes Dev. 30, 133-148.

Bestul, A.J., Yu, Z., Unruh, J.R., and Jaspersen, S.L. (2017). Molecular model of fission yeast centrosome assembly determined by superresolution imaging. J. Cell Biol. 216, 2409-2424.

Castillo, A.G., Mellone, B.G., Partridge, J.F., Richardson, W., Hamilton, G.L., Allshire, R.C., and Pidoux, A.L. (2007). Plasticity of fission yeast CENP-A chromatin driven by relative levels of histone H3 and H4. PLoS Genet. 3, e121.

Castillo, A.G., Pidoux, A.L., Catania, S., Durand-Dubief, M., Choi, E.S., Hamilton, G., Ekwall, K., and Allshire, R.C. (2013). Telomeric repeats facilitate CENP-A(Cnp1) incorporation via telomere binding proteins. PLoS One 8, e69673.

Catania, S., Pidoux, A.L., and Allshire, R.C. (2015). Sequence features and transcriptional stalling within centromere DNA promote establishment of CENP-A chromatin. PLoS Genet. 11, e1004986.

Chikashige, Y., Yamane, M., Okamasa, K., Tsutsumi, C., Kojidani, T., Sato, M., Haraguchi, T., and Hiraoka, Y. (2009). Membrane proteins Bqt3 and -4 anchor telomeres to the nuclear envelope to ensure chromosomal bouquet formation. J. Cell Biol. 187, 413-427.

Choi, E.S., Strålfors, A., Catania, S., Castillo, A.G., Svensson, J.P., Pidoux, A.L., Ekwall, K., and 
Allshire, R.C. (2012). Factors that promote H3 chromatin integrity during transcription prevent promiscuous deposition of CENP-A(Cnp1) in fission yeast. PLoS Genet. 8, e1002985.

Clarke, L., Baum, M., Marschall, L.G., Ngan, V.K., and Steiner, N.C. (1993). Structure and function of Schizosaccharomyces pombe centromeres. Cold Spring Harb. Symp. Quant. Biol. 58, 687-695.

DeBose-Scarlett, E.M., and Sullivan, B.A. (2021). Genomic and Epigenetic Foundations of Neocentromere Formation. Annu. Rev. Genet. 55, 331-348.

Ding, D.Q., and Hiraoka, Y. (2017). Visualization of a Specific Genome Locus by the lacO/Lacl-GFP System. Cold Spring Harb. Protoc. 10, pdb.prot091934.

Dunleavy, E.M., Almouzni, G., and Karpen, G.H. (2011). H3. 3 is deposited at centromeres in S phase as a placeholder for newly assembled CENP-A in G1 phase. Nucleus 2, 146-157.

Ebrahimi, H., Masuda, H., Jain, D., and Cooper, J.P. (2018). Distinct 'safe zones' at the nuclear envelope ensure robust replication of heterochromatic chromosome regions. Elife 7, e32911.

Fernández-Álvarez, A., Bez, C., O'Toole, E.T., Morphew, M., and Cooper, J.P. (2016). Mitotic Nuclear Envelope Breakdown and Spindle Nucleation Are Controlled by Interphase Contacts between Centromeres and the Nuclear Envelope. Dev. Cell 39, 544-559.

Folco, H.D., Pidoux, A.L., Urano, T., and Allshire, R.C. (2008). Heterochromatin and RNAi are required to establish CENP-A chromatin at centromeres. Science 319, 94-97.

Funabiki, H., Hagan, I., Uzawa, S., and Yanagida, M. (1993). Cell cycle-dependent specific positioning and clustering of centromeres and telomeres in fission yeast. J. Cell Biol. 121, 961-976. Gerlich, D., Beaudouin, J., Kalbfuss, B., Daigle, N., Eils, R., and Ellenberg, J. (2003). Global chromosome positions are transmitted through mitosis in mammalian cells. Cell 112, 751-764.

Guacci, V., Hogan, E., and Koshland, D. (1997). Centromere position in budding yeast: evidence for anaphase A. Mol. Biol. Cell 8, 957-972.

Hayashi, T., Fujita, Y., Iwasaki, O., Adachi, Y., Takahashi, K., and Yanagida, M. (2004). Mis16 and Mis18 are required for CENP-A loading and histone deacetylation at centromeres. Cell 118, 715-729. Hou, H., Zhou, Z., Wang, Y., Wang, J., Kallgren, S.P., Kurchuk, T., Miller, E.A., Chang, F., and Jia, S. (2012). Csi1 links centromeres to the nuclear envelope for centromere clustering. J. Cell Biol. 199, 735-744.

Iglesias, N., Paulo, J.A., Tatarakis, A., Wang, X., Edwards, A.L., Bhanu, N.V., Garcia, B.A., Haas, W., Gygi, S.P., and Moazed, D. (2020). Native Chromatin Proteomics Reveals a Role for Specific Nucleoporins in Heterochromatin Organization and Maintenance. Mol. Cell 77, 51-66.e58. 
Ishii, K., Ogiyama, Y., Chikashige, Y., Soejima, S., Masuda, F., Kakuma, T., Hiraoka, Y., and Takahashi, K. (2008). Heterochromatin integrity affects chromosome reorganization after centromere dysfunction. Science 321, 1088-1091.

Jaspersen, S.L. (2021). Anatomy of the fungal microtubule organizing center, the spindle pole body. Curr. Opin. Struct. Biol. 66, 22-31.

Kagansky, A., Folco, H.D., Almeida, R., Pidoux, A.L., Boukaba, A., Simmer, F., Urano, T., Hamilton, G.L., and Allshire, R.C. (2009). Synthetic heterochromatin bypasses RNAi and centromeric repeats to establish functional centromeres. Science $324,1716-1719$.

Kanoh, J., Sadaie, M., Urano, T., and Ishikawa, F. (2005). Telomere binding protein Taz1 establishes Swi6 heterochromatin independently of RNAi at telomeres. Curr. Biol. 15, 1808-1819.

Martienssen, R., and Moazed, D. (2015). RNAi and heterochromatin assembly. Cold Spring Harb. Perspect. Biol. 7, a019323.

Mellone, B.G., and Fachinetti, D. (2021). Diverse mechanisms of centromere specification. Curr. Biol. 31, R1491-R1504.

Mitra, S., Srinivasan, B., and Jansen, L.E.T. (2020). Stable inheritance of CENP-A chromatin: Inner strength versus dynamic control. J. Cell Biol. 219, e202005099.

Mizuguchi, T., Fudenberg, G., Mehta, S., Belton, J.M., Taneja, N., Folco, H.D., FitzGerald, P., Dekker, J., Mirny, L., Barrowman, J., and Grewal, S.I.S. (2014). Cohesin-dependent globules and heterochromatin shape 3D genome architecture in S. pombe. Nature 516, 432-435.

Moreno, S., Klar, A., and Nurse, P. (1991). Molecular genetic analysis of fission yeast Schizosaccharomyces pombe. Methods Enzymol. 194, 795-823.

Muller, H., Gil, J., Jr., and Drinnenberg, I.A. (2019). The Impact of Centromeres on Spatial Genome Architecture. Trends Genet. 35, 565-578.

Noma, K., Cam, H.P., Maraia, R.J., and Grewal, S.I. (2006). A role for TFIIIC transcription factor complex in genome organization. Cell 125, 859-872.

Ogiyama, Y., Ohno, Y., Kubota, Y., and Ishii, K. (2013). Epigenetically induced paucity of histone H2A.Z stabilizes fission-yeast ectopic centromeres. Nat. Struct. Mol. Biol. 20, 1397-1406.

Oko, Y., Ito, N., and Sakamoto, T. (2020). The mechanisms and significance of the positional control of centromeres and telomeres in plants. J. Plant Res. 133, 471-478.

Pichugina, T., Sugawara, T., Kaykov, A., Schierding, W., Masuda, K., Uewaki, J., Grand, R.S., Allison, J.R., Martienssen, R.A., Nurse, P., et al. (2016). A diffusion model for the coordination of DNA 
replication in Schizosaccharomyces pombe. Sci. Rep. 6, 18757.

Pidoux, A.L., Choi, E.S., Abbott, J.K., Liu, X., Kagansky, A., Castillo, A.G., Hamilton, G.L., Richardson, W., Rappsilber, J., He, X., and Allshire, R.C. (2009). Fission yeast Scm3: A CENP-A receptor required for integrity of subkinetochore chromatin. Mol. Cell 33, 299-311.

Rhind, N., Chen, Z., Yassour, M., Thompson, D.A., Haas, B.J., Habib, N., Wapinski, I., Roy, S., Lin, M.F., Heiman, D.I., et al. (2011). Comparative functional genomics of the fission yeasts. Science 332, 930-936.

Saitoh, S., Takahashi, K., and Yanagida, M. (1997). Mis6, a fission yeast inner centromere protein, acts during G1/S and forms specialized chromatin required for equal segregation. Cell 90, 131-143. Scott, K.C., Merrett, S.L., and Willard, H.F. (2006). A heterochromatin barrier partitions the fission yeast centromere into discrete chromatin domains. Curr. Biol. 16, 119-129.

Shukla, M., Tong, P., White, S.A., Singh, P.P., Reid, A.M., Catania, S., Pidoux, A.L., and Allshire, R.C. (2018). Centromere DNA Destabilizes H3 Nucleosomes to Promote CENP-A Deposition during the Cell Cycle. Curr. Biol. 28, 3924-3936.e4.

Singh, P.P., Shukla, M., White, S.A., Lafos, M., Tong, P., Auchynnikava, T., Spanos, C., Rappsilber, J., Pidoux, A.L., and Allshire, R.C. (2020). Hap2-Ino80-facilitated transcription promotes de novo establishment of CENP-A chromatin. Genes Dev. 34, 226-238.

Stankovic, A., Guo, L.Y., Mata, J.F., Bodor, D.L., Cao, X.J., Bailey, A.O., Shabanowitz, J., Hunt, D.F., Garcia, B.A., Black, B.E., and Jansen, L.E.T. (2017). A Dual Inhibitory Mechanism Sufficient to Maintain Cell-Cycle-Restricted CENP-A Assembly. Mol. Cell 65, 231-246.

Steiner, N.C., and Clarke, L. (1994). A novel epigenetic effect can alter centromere function in fission yeast. Cell 79, 865-874.

Subramanian, L., Toda, N.R., Rappsilber, J., and Allshire, R.C. (2014). Eic1 links Mis18 with the CCAN/Mis6/Ctf19 complex to promote CENP-A assembly. Open Biol. 4, 140043.

Takahashi, K., Murakami, S., Chikashige, Y., Funabiki, H., Niwa, O., and Yanagida, M. (1992). A low copy number central sequence with strict symmetry and unusual chromatin structure in fission yeast centromere. Mol. Biol. Cell 3, 819-835.

Tong, P., Pidoux, A.L., Toda, N.R.T., Ard, R., Berger, H., Shukla, M., Torres-Garcia, J., Müller, C.A., Nieduszynski, C.A., and Allshire, R.C. (2019). Interspecies conservation of organisation and function between nonhomologous regional centromeres. Nat. Commun. 10, 2343.

Torres-Garcia, S., Di Pompeo, L., Eivers, L., Gaborieau, B., White, S.A., Pidoux, A.L., Kanigowska, 
P., Yaseen, I., Cai, Y., and Allshire, R.C. (2020). SpEDIT: A fast and efficient CRISPR/Cas9 method for fission yeast. Wellcome Open Res. 5, 274.

Westhorpe, F.G., and Straight, A.F. (2015). The centromere: epigenetic control of chromosome segregation during mitosis. Cold Spring Harb. Perspect. Biol. 7, a015818.

Williams, J.S., Hayashi, T., Yanagida, M., and Russell, P. (2009). Fission yeast Scm3 mediates stable assembly of Cnp1/CENP-A into centromeric chromatin. Mol. Cell 33, 287-298.

Winey, M., and O'Toole, E.T. (2001). The spindle cycle in budding yeast. Nat. Cell Biol. 3, E23-27.

Zasadzińska, E., and Foltz, D.R. (2017). Orchestrating the Specific Assembly of Centromeric Nucleosomes. Prog. Mol. Subcell. Biol. 56, 165-19. 


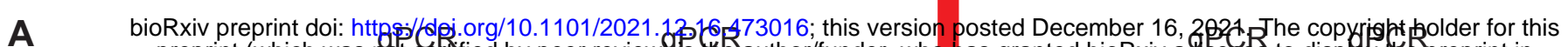
preprint (which was not ceftified by peer review) ts the author/funder, who has granted bioRxiv a flcense to display the preprint in anperpetuity. It is made aveitable under aCC-BY-NC-ND 4.0 International lic đin'se.

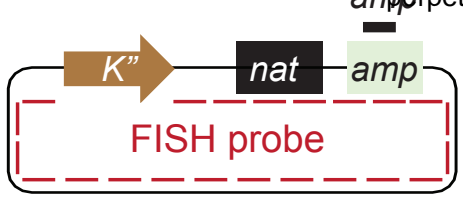

pHet

B

qChIP: H3K9me2

amp dg act1

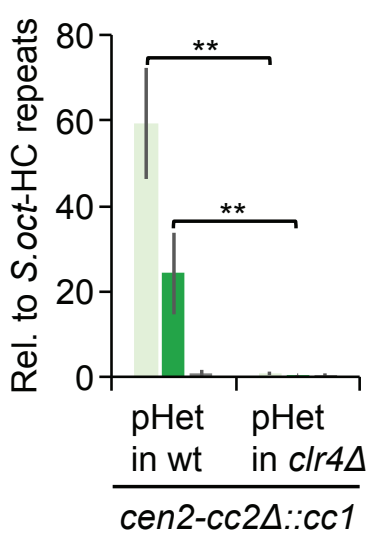

C

qChIP: CENP-A ${ }^{\text {Cnp1 }}$

cc2 cc1/3act1

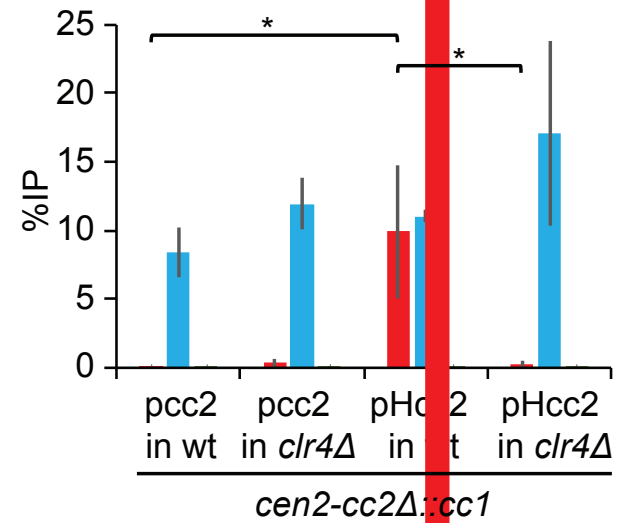

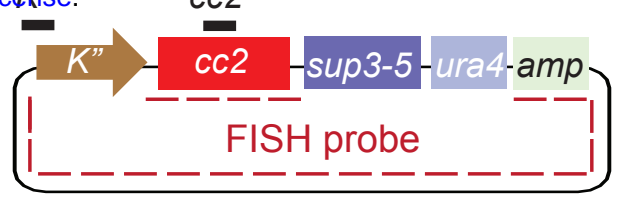

pHcc2

D

qChIP: H3K9me2

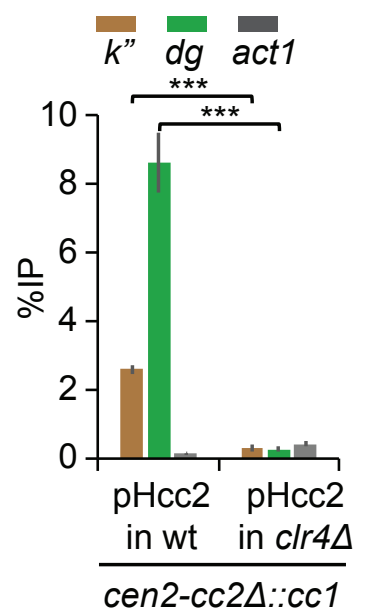

E

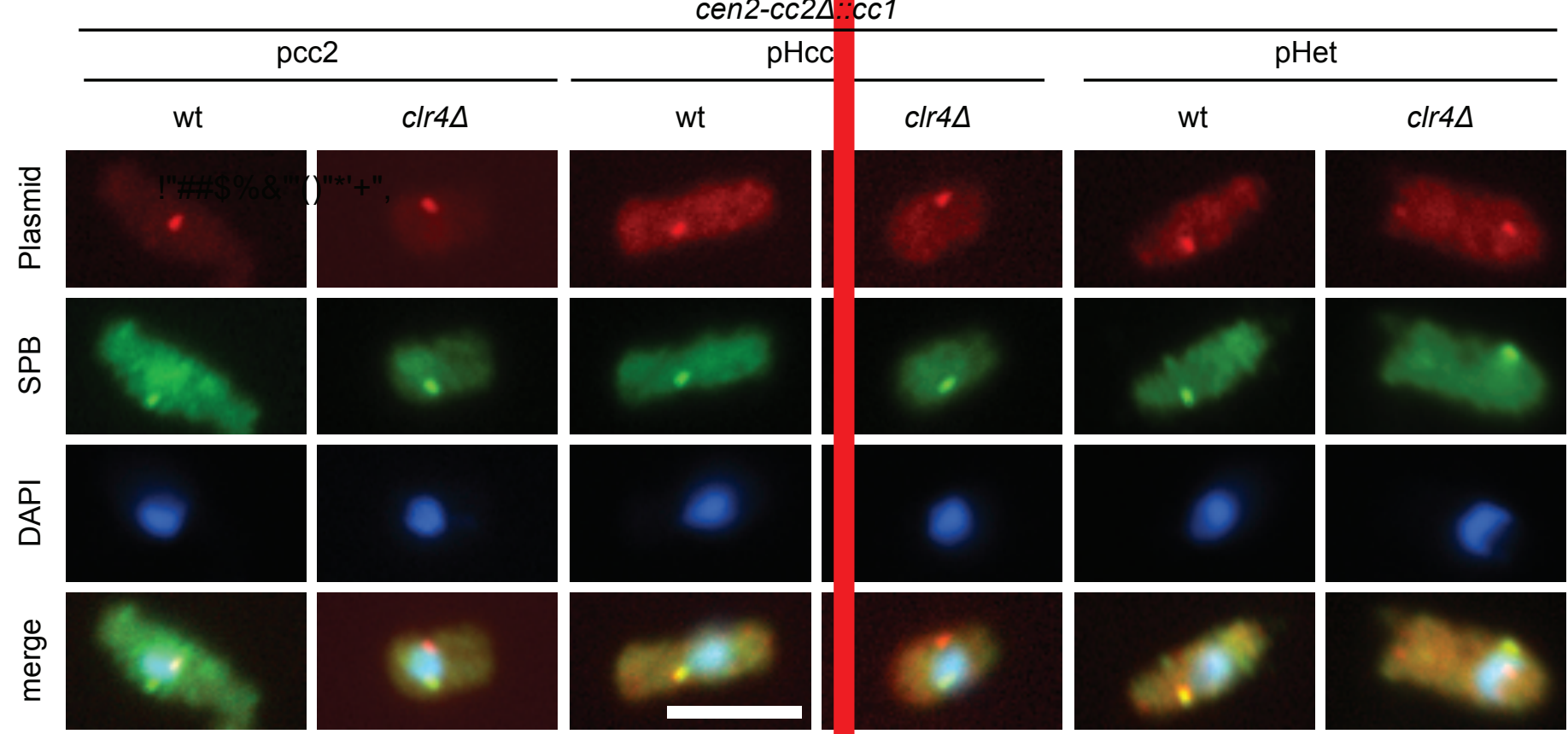

cen2-cc2 $\triangle:: c c 1$

$\mathbf{F}$

plasmid-SPB Co-localisation

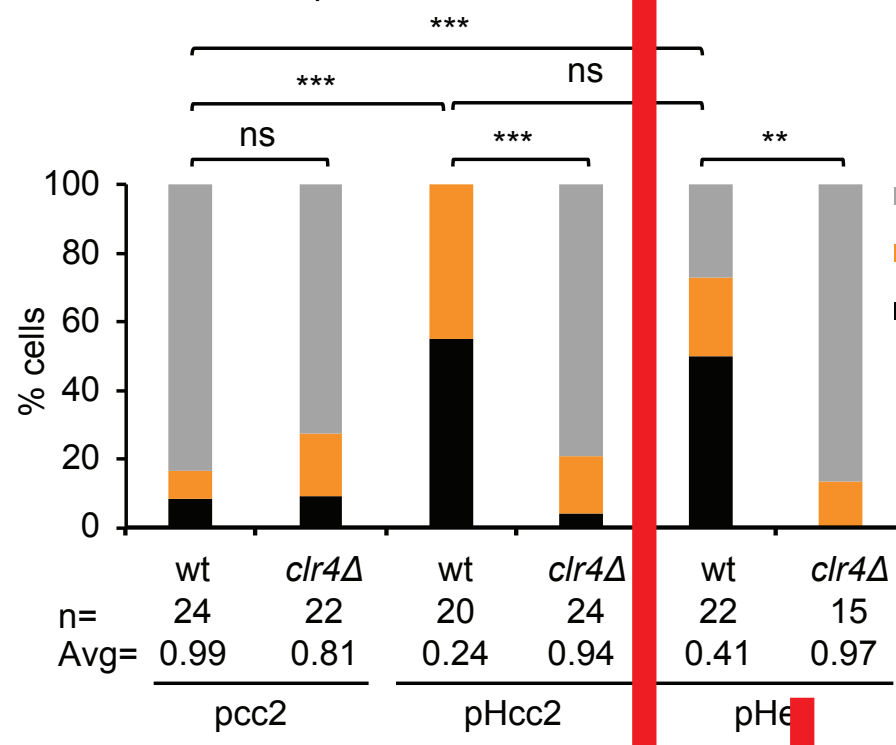

separate

- adjacent

- overlap 
bioRxiv preprint doi: https://doi.org/10.1101/2021.12.16.473016; this version posted December 16, 2021. The copyright holder for this A preprint (which was not certified by peer review) is the author/funder, who has granted bioRxiv a license to display the preprint in Chrl perpetuity. It is made available under aCC-BY-NC-NQ 4.9 International license.

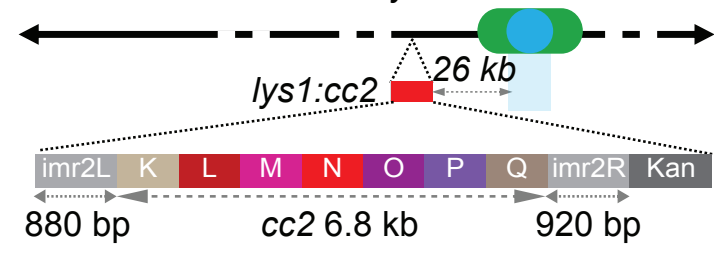

B
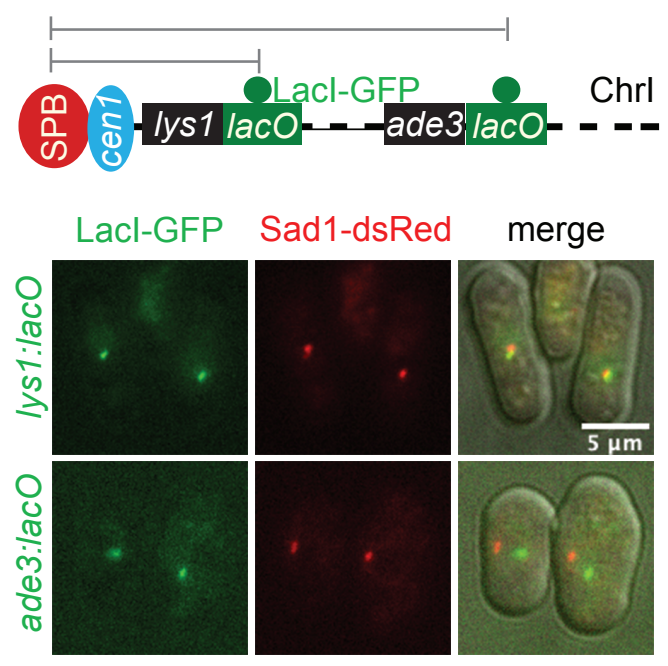

Sad1-dsRed
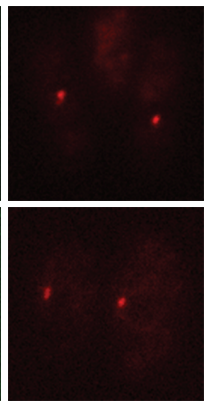

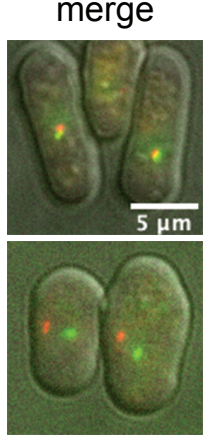

C

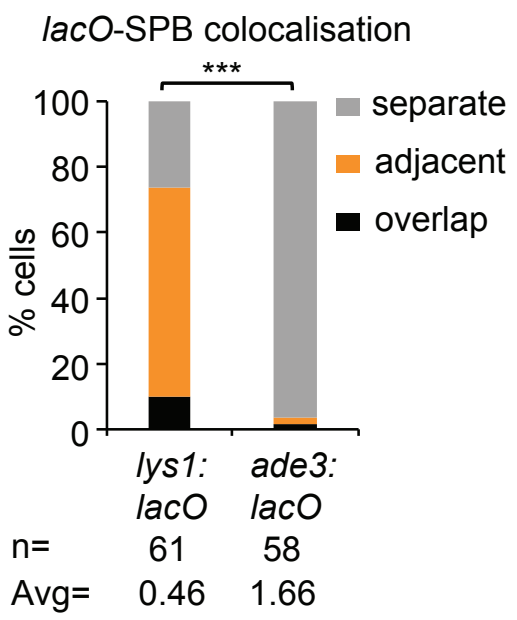

D

qChIP: CENP-A ${ }^{\text {Cnp1 }}$

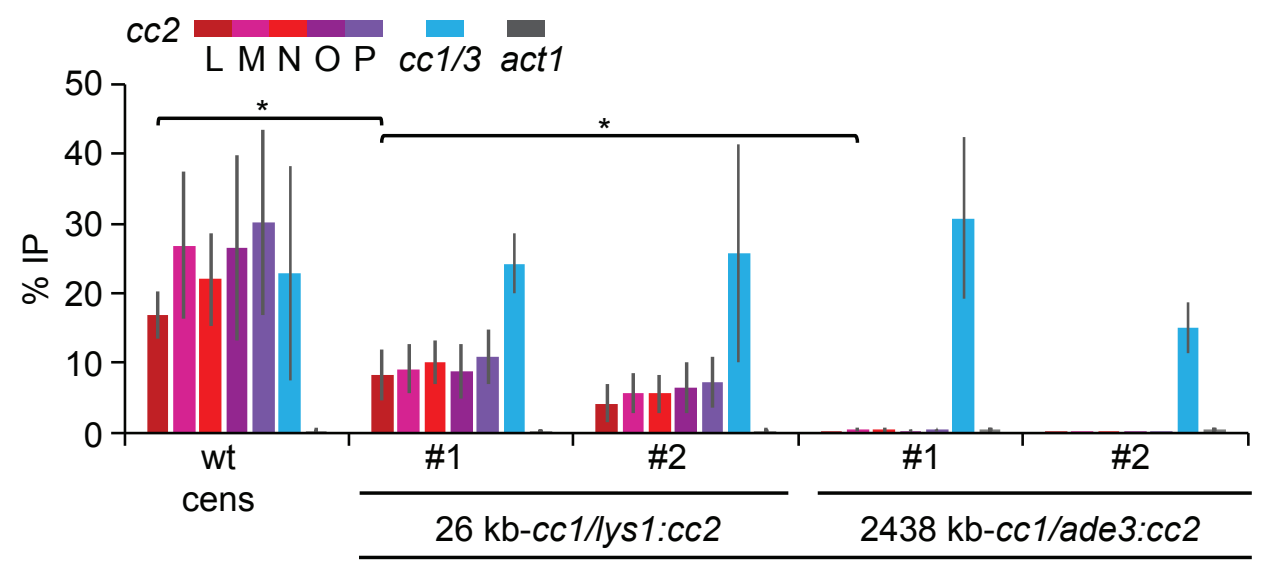

cen2-cc2 $\triangle:: c c 1$

E

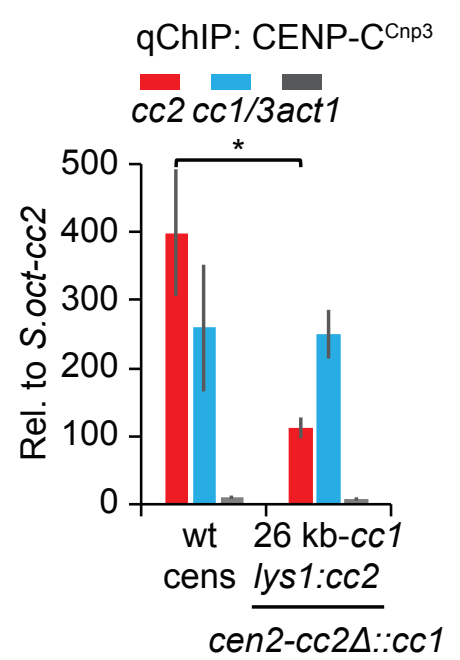

F

qChIP: CENP-K ${ }^{\operatorname{sim} 4}$ cc2 $c c 1 / 3 a c t 1$

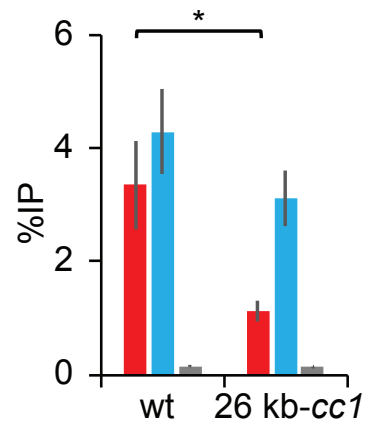

cens lys1:cc2

cen2-cc2 $\triangle:: c c 1$
G

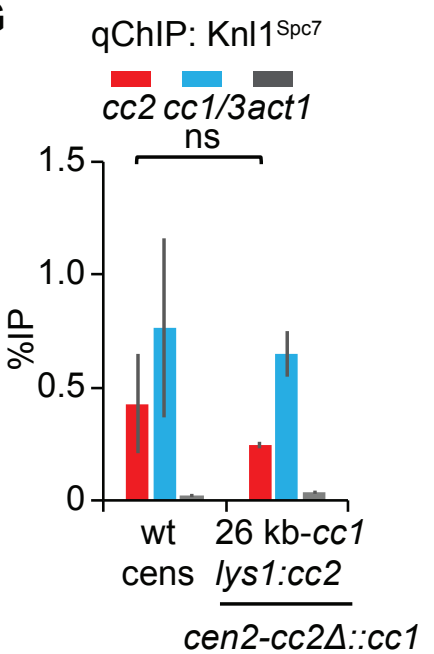

Wu et al., Figure 2 
bioRxiv preprint doi: https://doi.org/10.1101/2021.12.16.473016; this version posted December 16,2021 . The copyright holder for this preprint (which was not certified by peer review) is the author/funder, who has granted bioRxiv a license to display the preprint in perpetuity. It is made available under aCC-BY-NC-ND 4.0 International license.

A
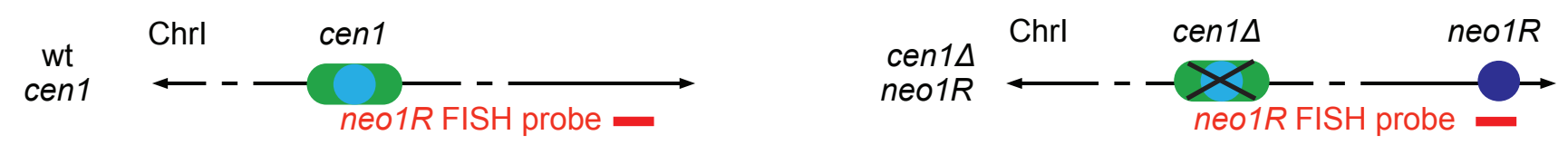

B

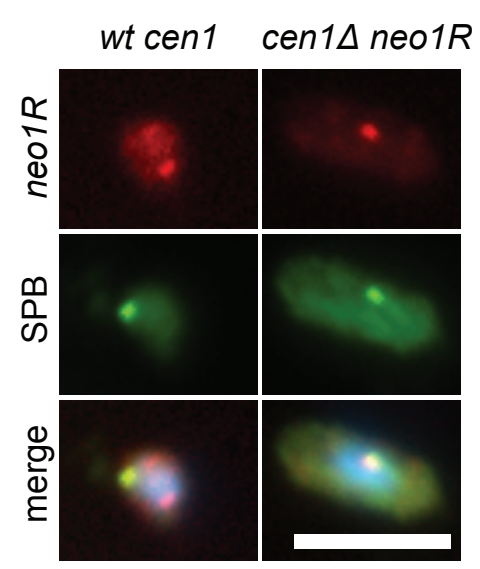

C

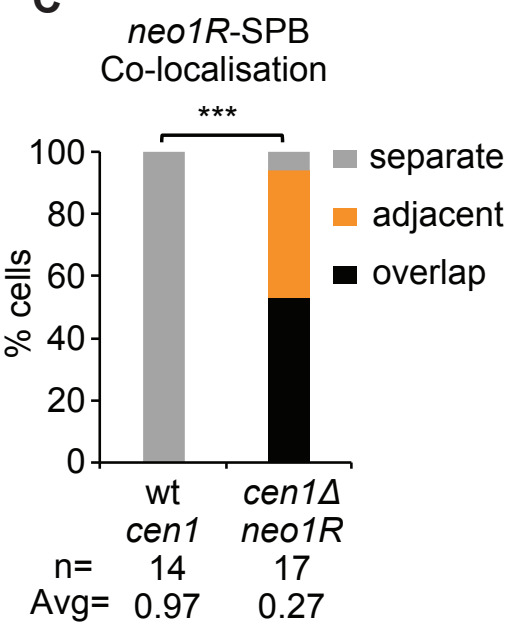

D

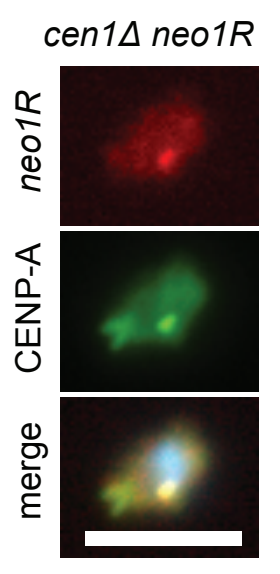

E

neo1R-CENP-A

Co-localisation

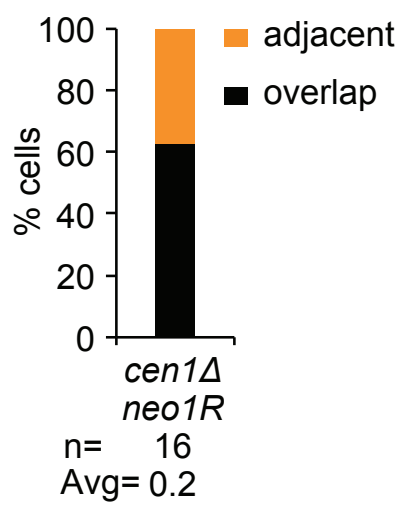

Wu et al., Figure3 
A preprint (which was not certified by peer review) is the author/funder, who has granted bioRxiv a license to display the preprint in

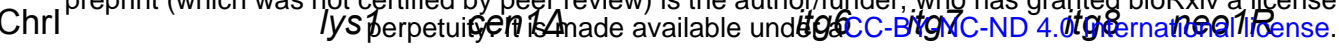

B

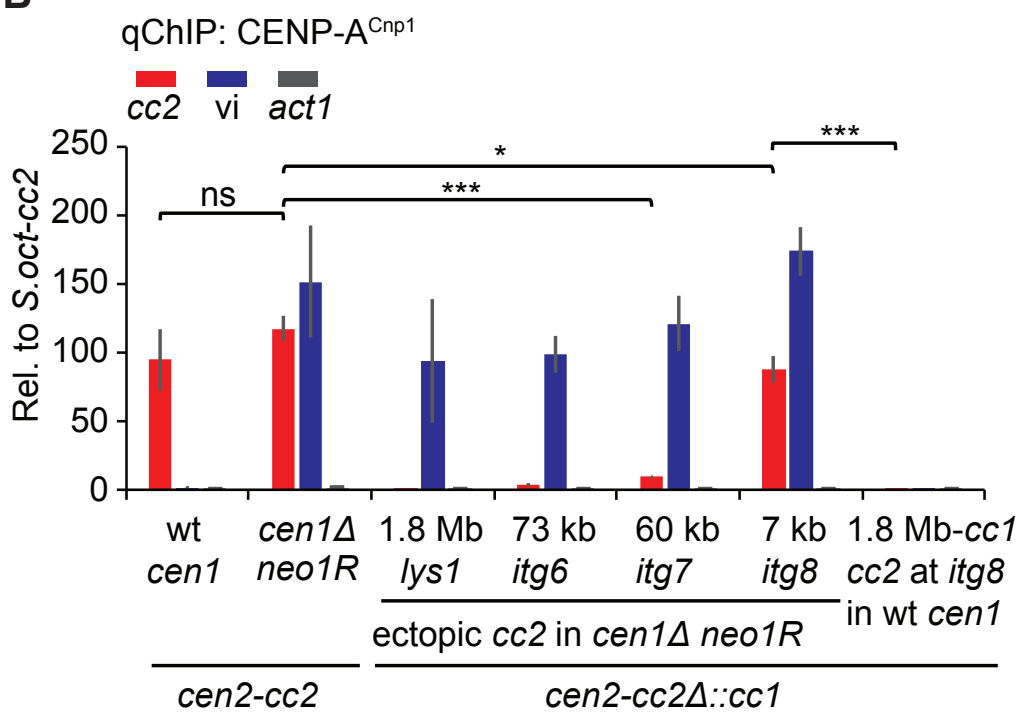

F

qChIP: CENP-A ${ }^{\text {Cnp } 1}$

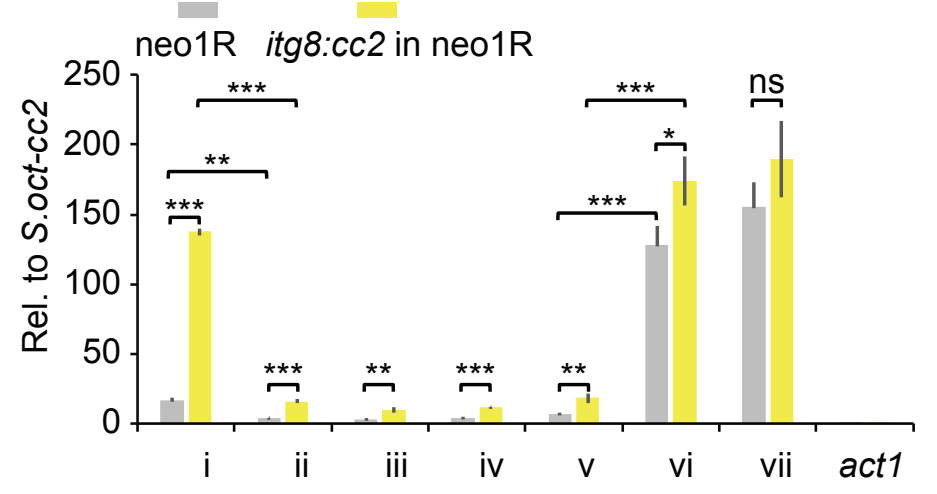

C
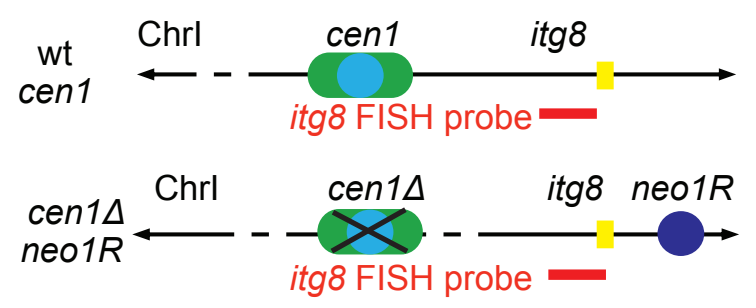

D

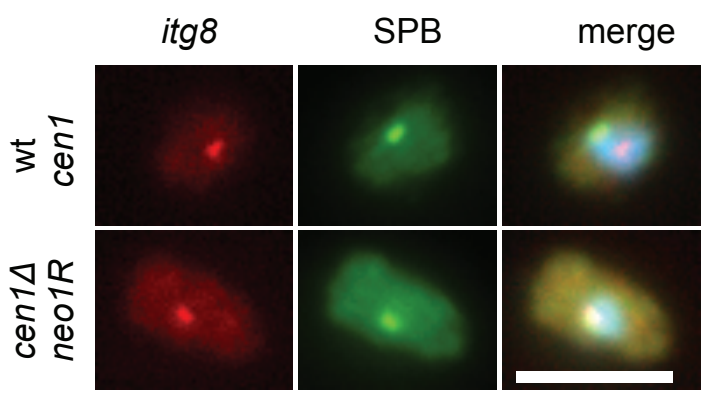

E

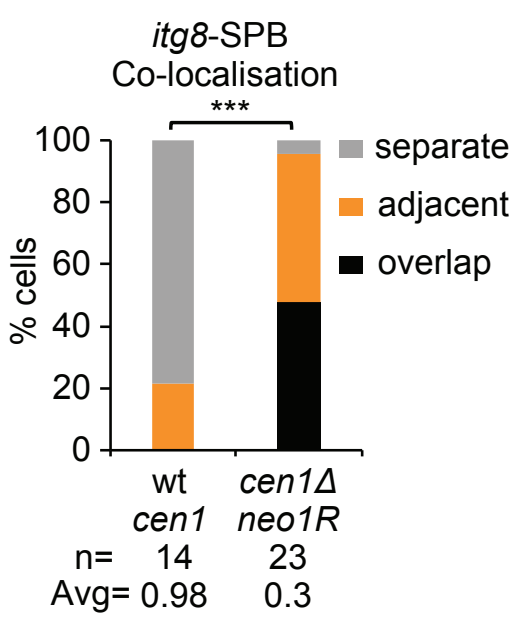

Wu et al., Figure 4 
A bioRxiv preprint doi: https://doi.org/10.1101/2021.12.16.473016; this grsion posted December 16, 2021. The copyright holder for this Lemeprint (which wasnot certified by peer review) is the author/fundeP, who has granted bioRxiv a license to display the preprintigitoplasm
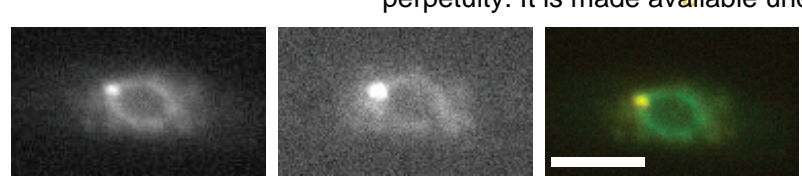

Lacl-GFP

Lem2-GBP-mCherry

merge
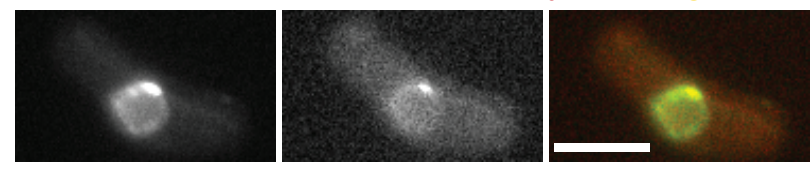

C

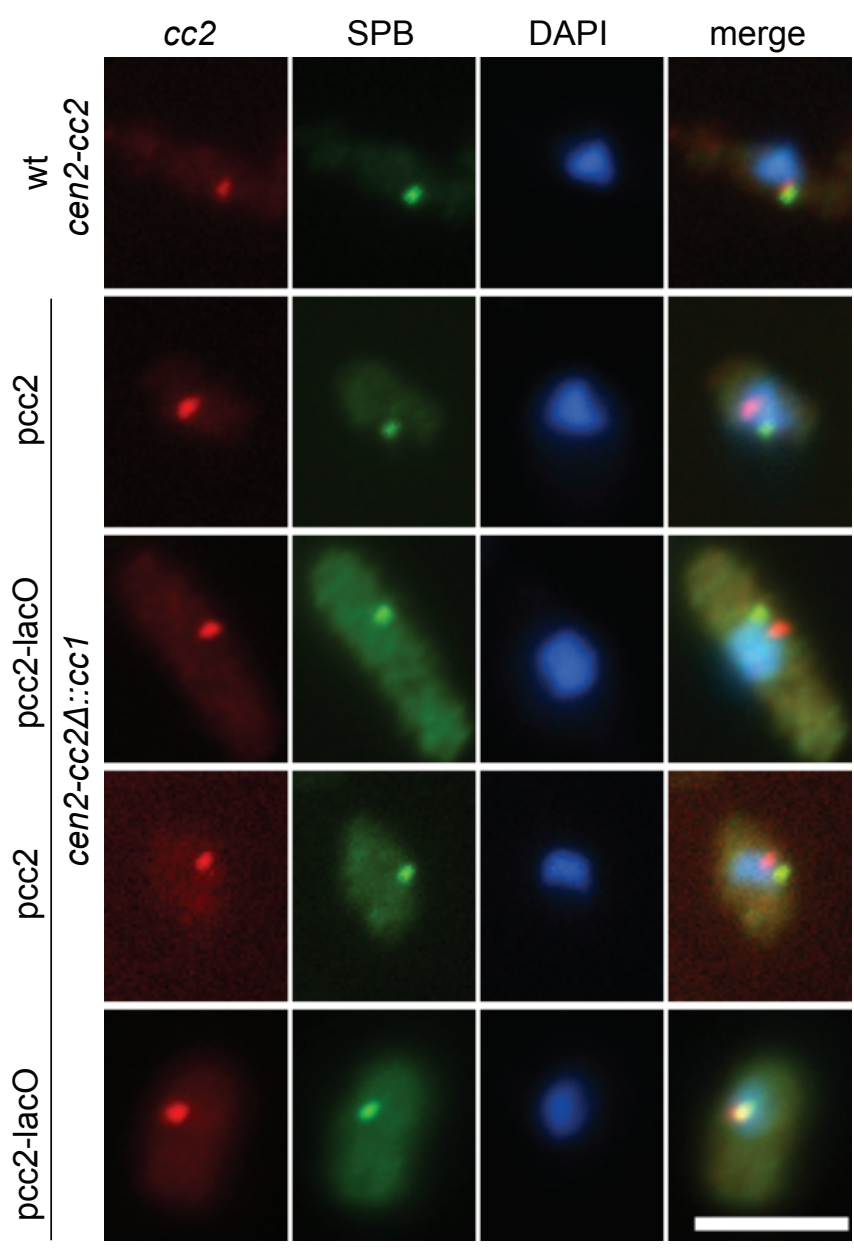

D

cc2-SPB colocalisation

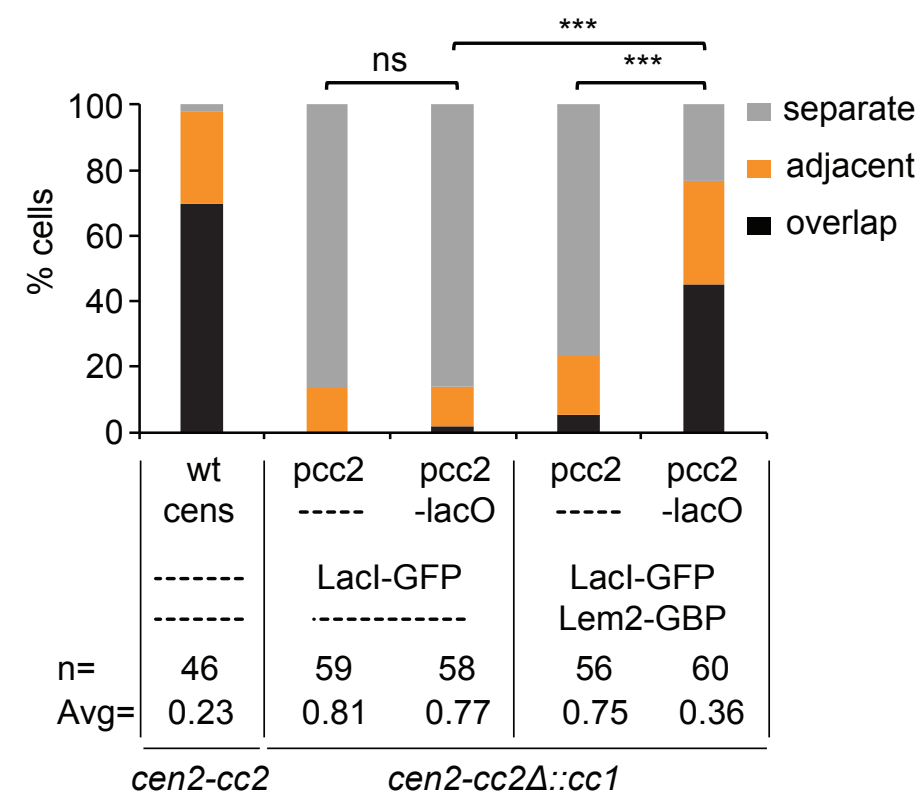

E

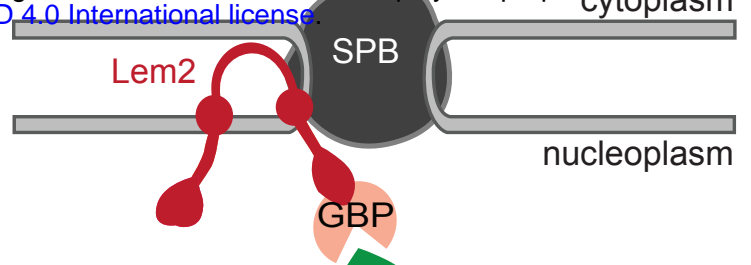

pcc2-lacO
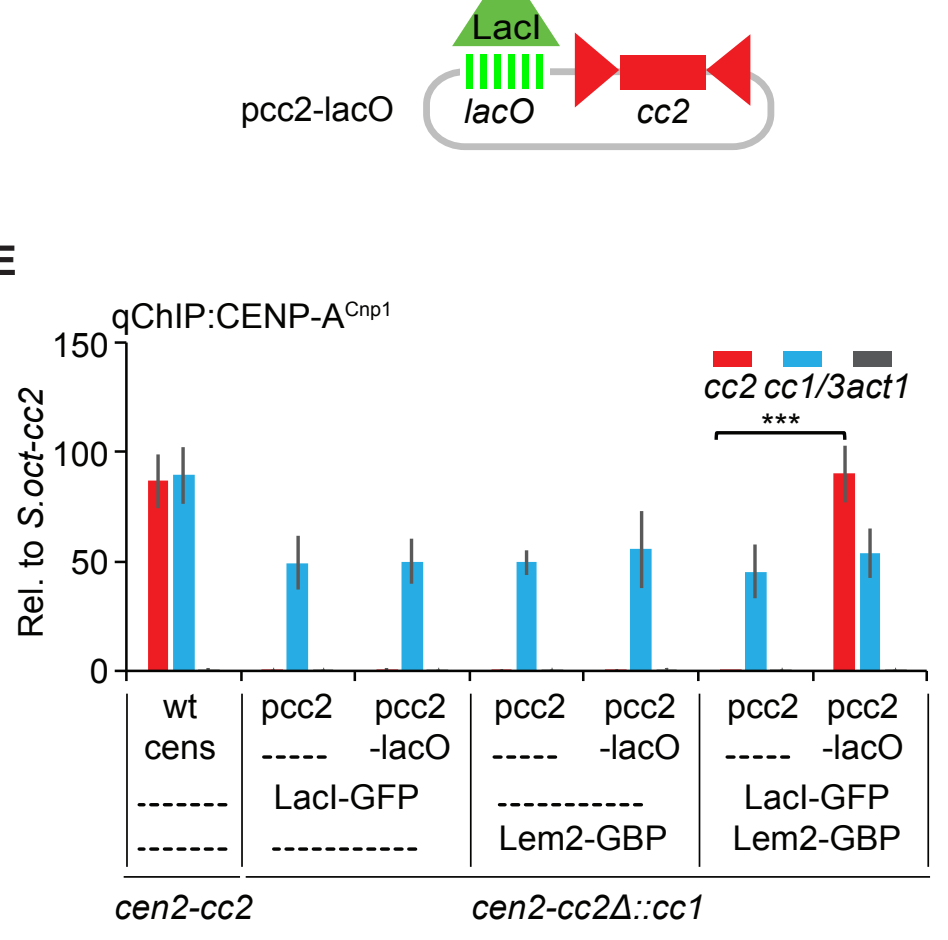

F

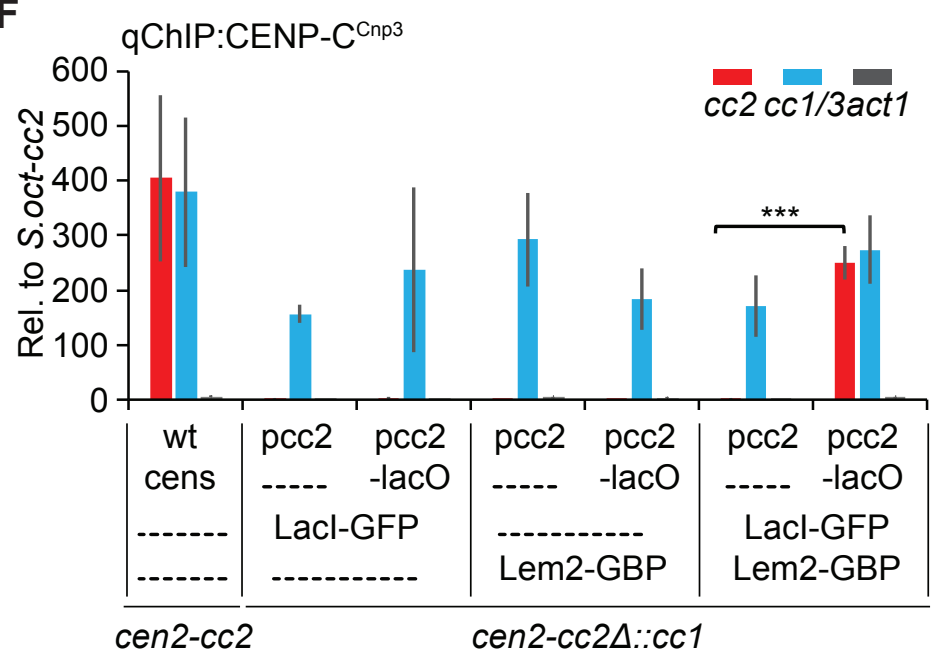

G

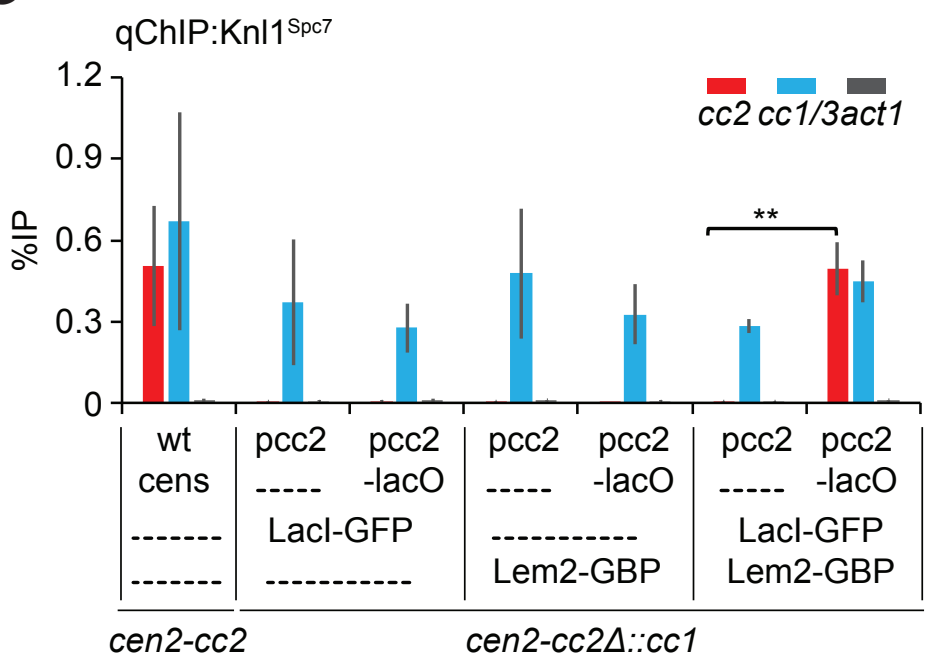

Wu et al., Figure 5 
bioRxiv preprint doi: https://doi.org/10.1101/2021.12.16.473016; this version posted December 16, 2021. The copyright holder for this

preprint (which was not certified by peer review) is the author/funder, who has granted bioRxiv a license to display the preprint in

A
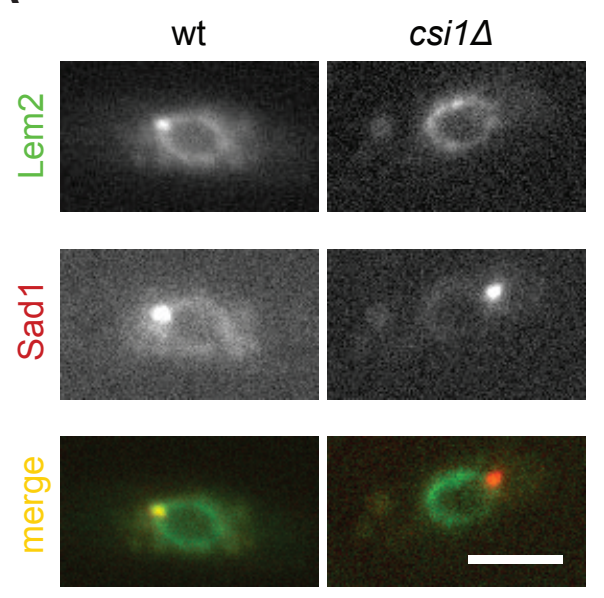

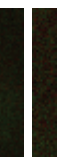

B

D

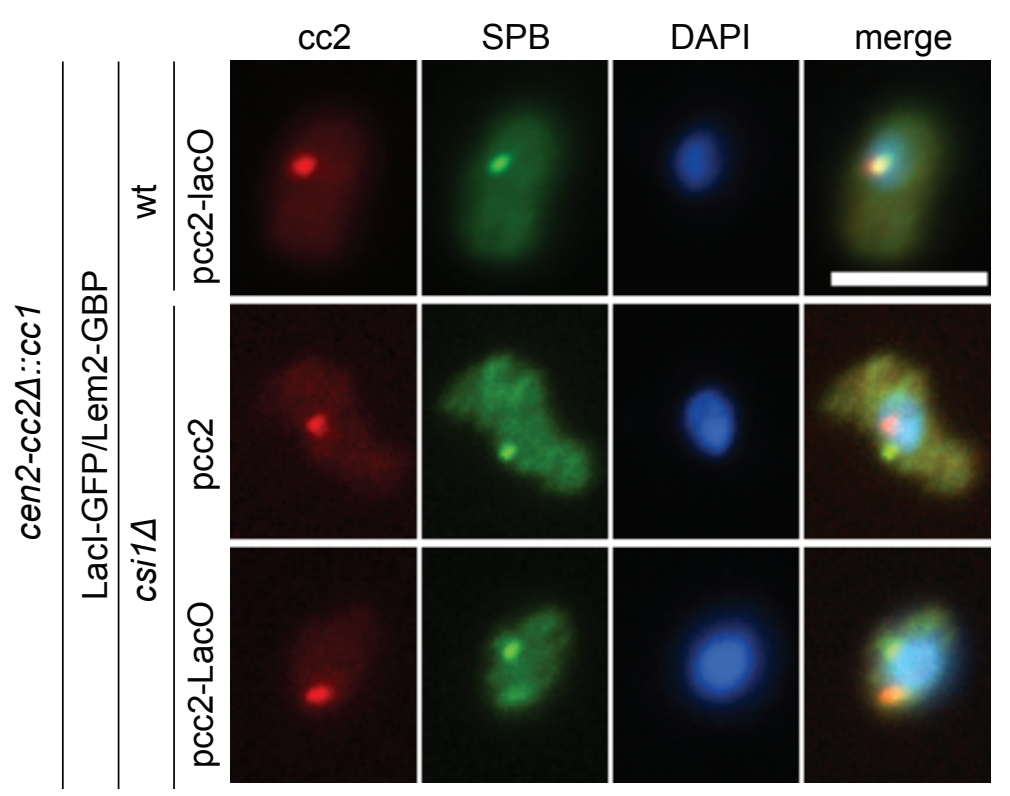

$\mathbf{F}$

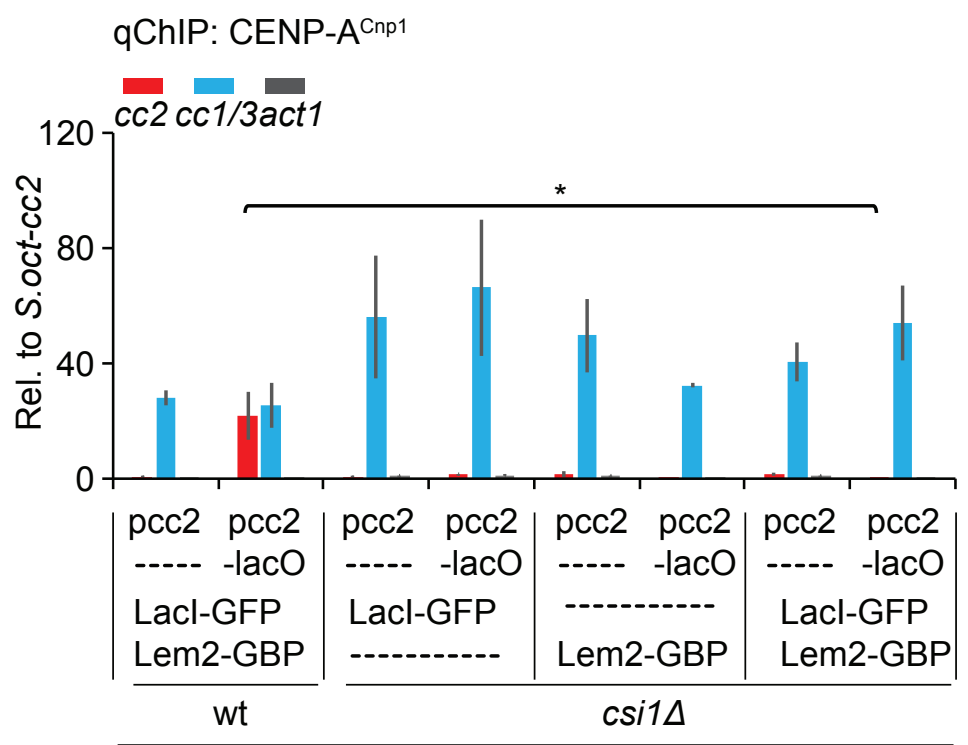

cen2-cc2 $\Delta:: c c 1$
C
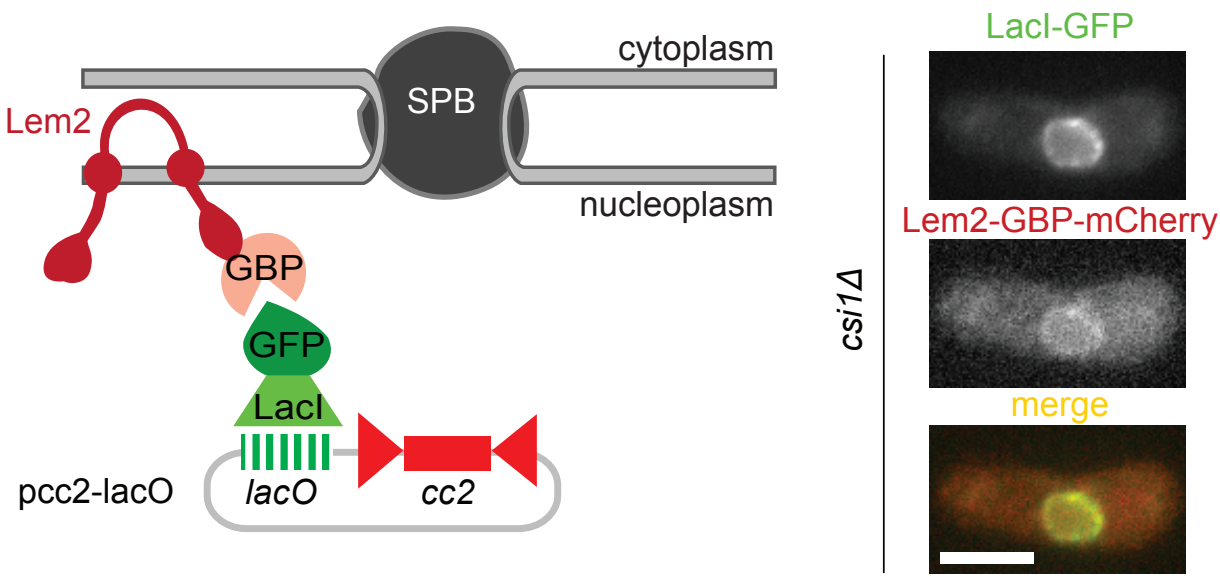

E

cc2-SPB Co-localisation

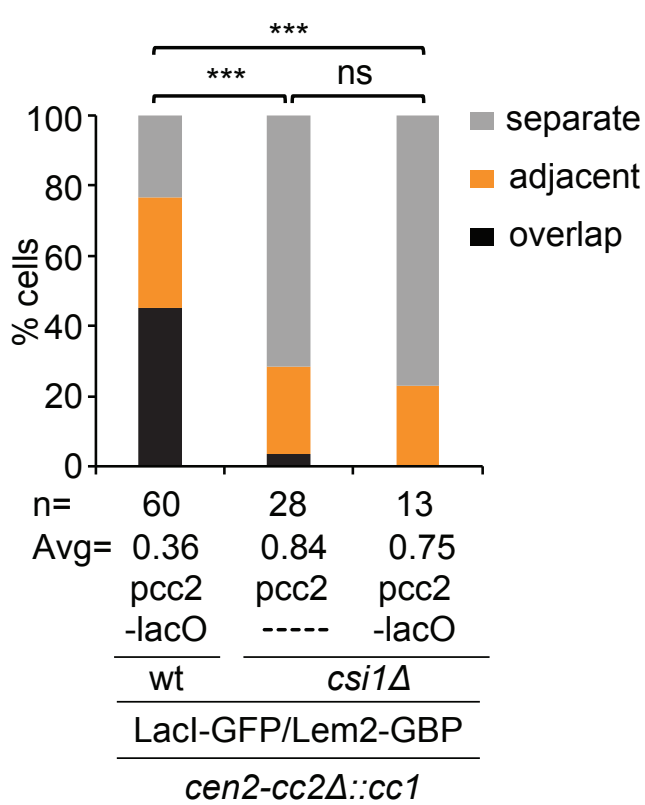

G
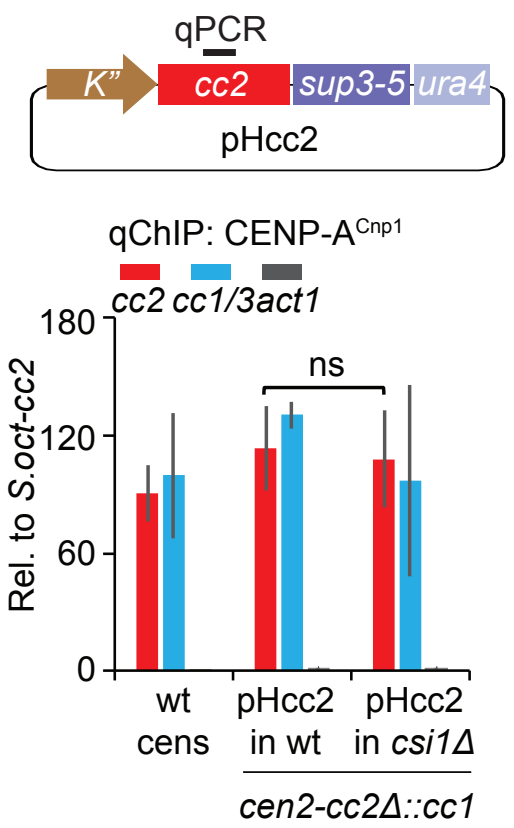

Wu et al., Figure 6 
bioRxiv preprint doi: https://doi.org/10.1101/2021.12.16.473016; this version posted December 16, 2021. The copyright holder for this preprint (which was not certified by peer review) is the author/funder, who has granted bioRxiv a license to display the preprint in perpetuity. It is made available under aCC-BY-NC-ND 4.0 International license.

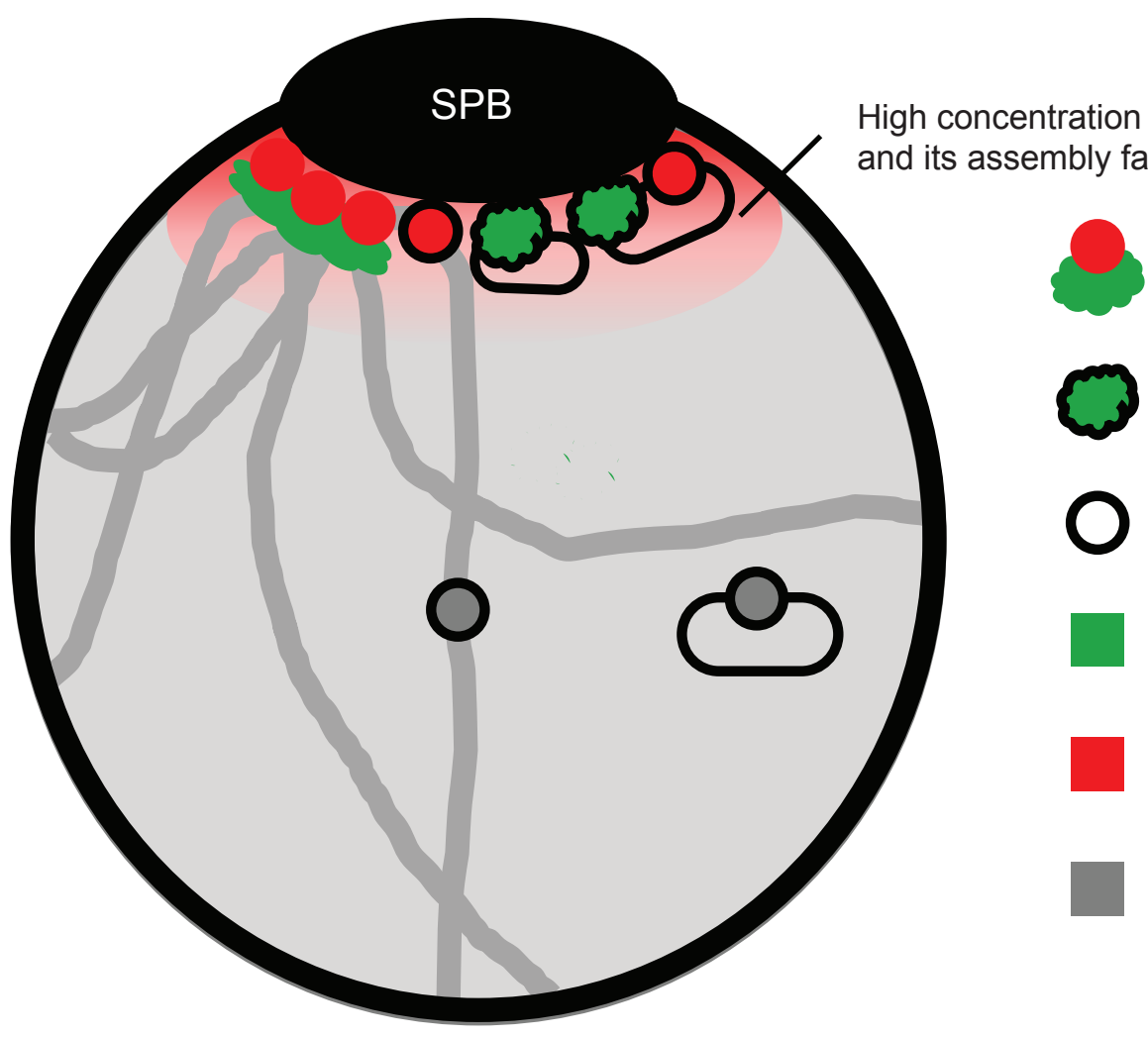

endogenous centromere

plamsid-boren outer repeat assembled in heterochromatin ectopic or plasmid-borne cc2, can be assembled in $\mathrm{H} 3$ or CENP-A ${ }^{\text {Cnp1 }}$ chromatin

heterochromatin

CENP-A ${ }^{\text {Cnp1 }}$ chromatin

H3 chromatin

Wu et al., Figure 7 
A wt cens strain:
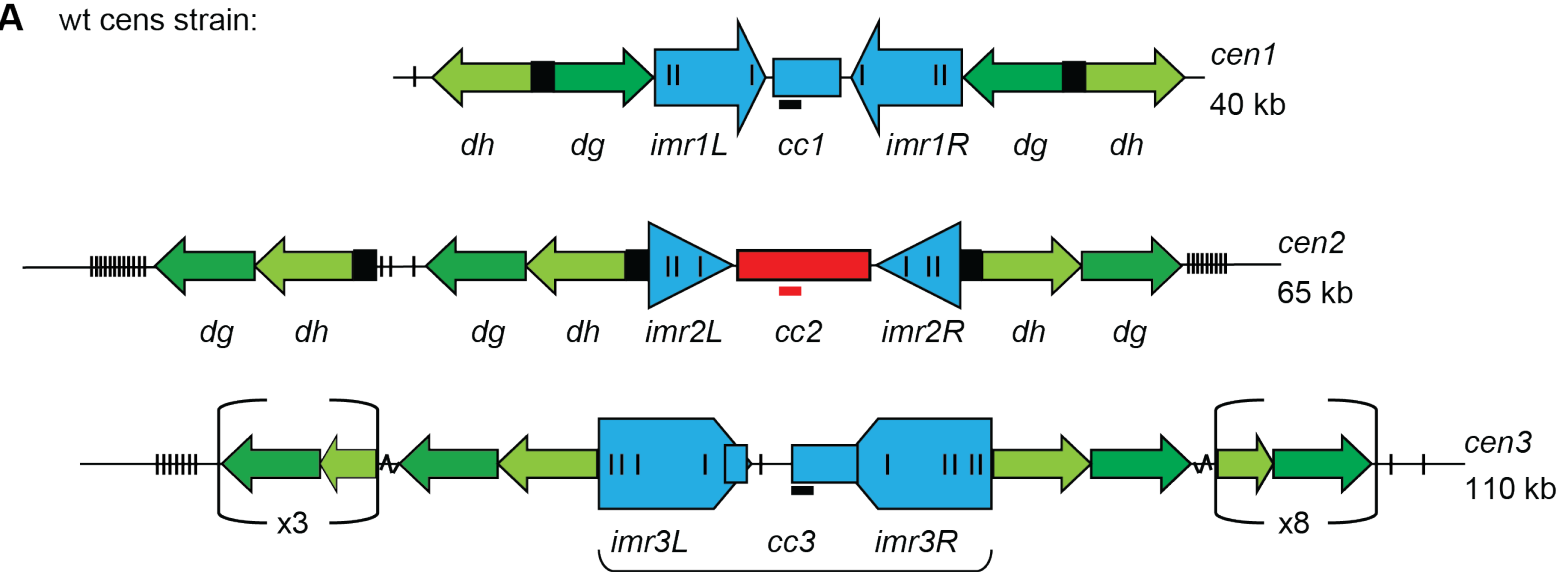

Outer Repeats (otr) H3K9me heterochromatin

Central Domain CENP-A ${ }^{\text {Cnp1 }}$ chromatin
Outer Repeats (otr) $\mathrm{H} 3 \mathrm{~K} 9 \mathrm{me}$ heterochromatin

B $\quad c c 2 \Delta:: c c 1$ strain:
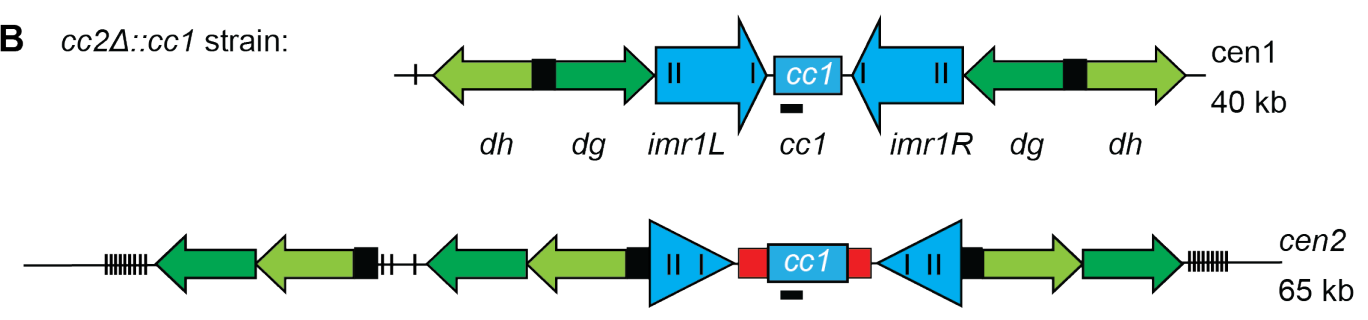

$d g \quad d h \quad d g \quad d h \quad$ imr2L $c c 2 \Delta:: c c 1$ imr2R $d h \quad d g$

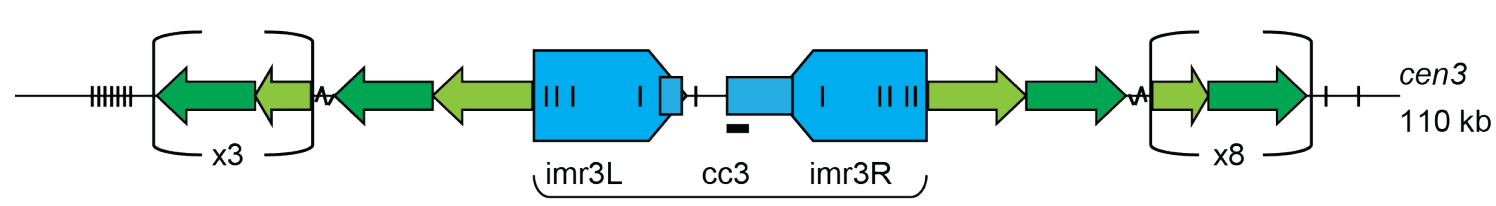

Outer Repeats (otr) H3K9me heterochromatin

Central Domain

chromatin
Outer Repeats (otr) H3K9me heterochromatin

Figure S1. Domain organization of centromeres in fission yeast. Relates to Figures 1-7

(A, B) Schematic representation of the three endogenous centromeres in wt-cens (A) or $c c 2 \Delta:: c c 1$ strains (B). Each centromere consists of two distinct domains: a central domain assembled in CENP-A ${ }^{\text {Cnp1 }}$ chromatin harbouring a central core $(c c)$ DNA and flanking innermost repeats $(\mathrm{imr})$, which are surrounded by various repetitive DNA elements known as the outer repeats (otr-dg/dh) assembled in H3K9me-dependent heterochromatin (Allshire and Ekwall, 2015). In $c c 2 \Delta:: c c 1$ cells, $6 \mathrm{~kb}$ of $c c 2$ DNA was replaced by $5.5 \mathrm{~kb}$ of $c c 1$ sequence, allowing the specific analysis of unique $c c 2$ DNA present in ectopic $c c 2$ insertions and minichromosomes pcc2 and pHcc2 (Catania et al., 2015). Black, red bars represent qChIP primer sites on $c c 1 / 3$ (the homologous region between $c c 1$ and $c c 3$ ) and cc2 DNA, respectively. 
A

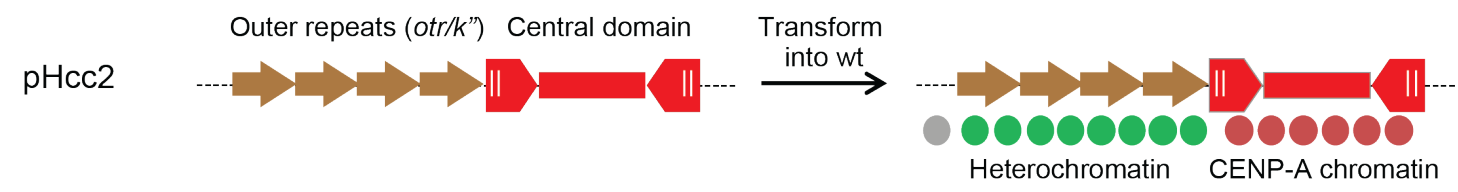

B

pHcc2
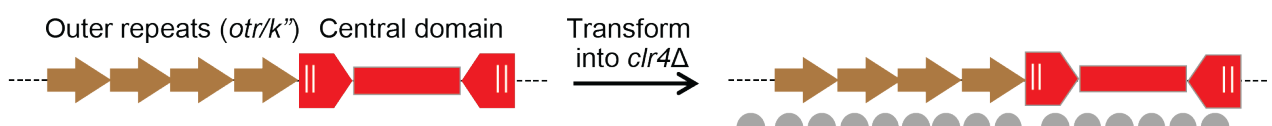

00000000000

C

$\mathrm{pHcc} 2$

D

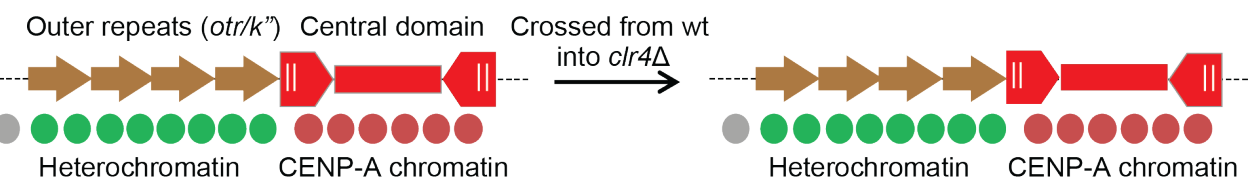

pcc2

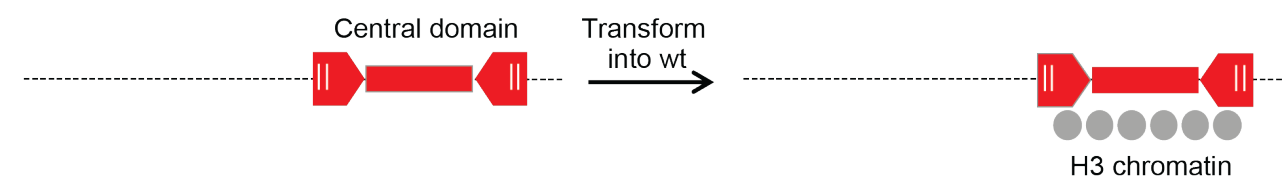

E

pcc2

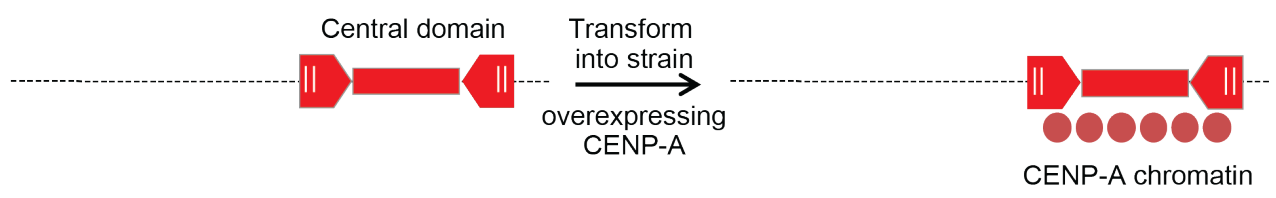

F
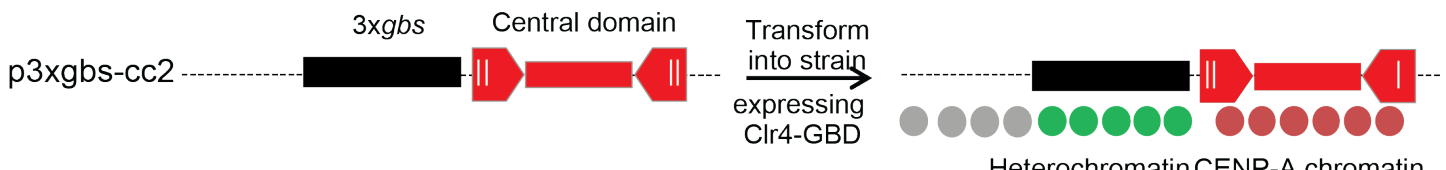

H3

H3K9me

CENP-A

Figure S2. Heterochromatin is required for CENP-A ${ }^{\text {Cnp1 }}$ chromatin establishment unless CENP-A ${ }^{\text {Cnp1 }}$ is overexpressed. Relates to Figure 1

(A, B) pHcc2 assembles heterochromatin on outer repeats (otr/K") which promotes CENP$A^{\text {Cnp1 }}$ chromatin establishment on its central domain DNA in wt (A) cells but not in clr4 $\triangle(B)$ cells following transformation (Folco et al., 2008).

(C) Heterochromatin is not required to maintain CENP-A ${ }^{\text {Cnp1 }}$ chromatin on central domain of pHcc2 in clr4 $\Delta$ crossed from wt (Folco et al., 2008).

$(D, \quad E)$ pcc2 assembles CENP-A ${ }^{\text {Cnp1 }}$ chromatin on its central domain in CENP$A^{\text {Cnp1 }}$ overexpressed cells (E) but not in wt CENP-A ${ }^{\text {Cnp1 }}$ cells (D) upon transformation (Catania et al., 2015).

(F) p3xgbs-cc2 assembles heterochromatin on 3xgbs (three Gal4-binding sites) that permits CENP-A ${ }^{\text {Cnp1 }}$ chromatin establishment on its $c c 2$ central domain in cells expressing Clr4GBD (the DNA binding domain of the S. cerevisiae Gal4 protein) fusions following transformation. Artificial association of Clr4 with $3 x g b s$ bypasses the requirement for heterochromatic outer repeats (otr/K') in heterochromatin assembly (Kagansky et al., 2009). 
A

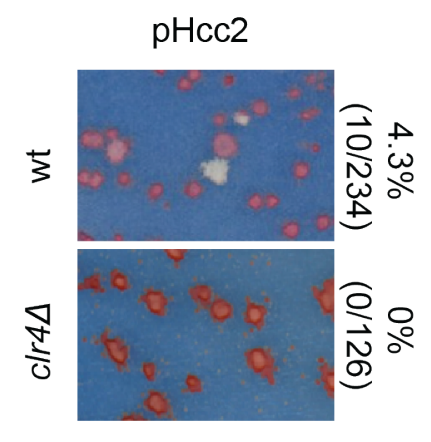

B

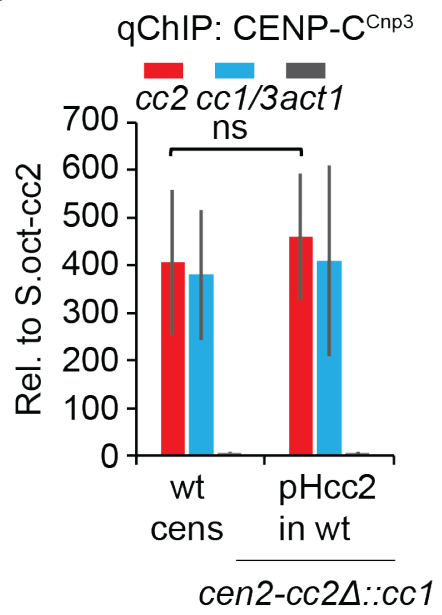

C

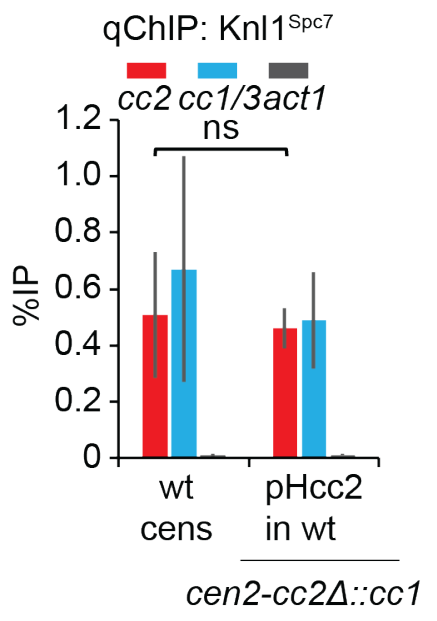

Figure S3. Kinetochore proteins are recruited to minichromosome $\mathrm{pHcc2}$ transformed into wt cells. Relates to Figure 1

(A) Functional centromere establishment assay on pHcc2 transformed into wt and clr4 $\Delta$ strains carrying $c c 2 \Delta:: c c 1$. Transformants were replica plated to low adenine non-selective plate. Pale pink/white colonies indicate the presence of functional centromere since sup3e tRNA on pHcc2 suppresses ade6-704 mutation within strains. Establishment frequency was calculated as the percentage of pale pink/white colonies divided by the total number of transformants (See also STAR Methods), thus $4.3 \%, 0 \%$ of colonies established functional centromeres on pHcc2 transformed into wt and clr $4 \Delta$ cells, respectively.

$(B, C)$ qChIP analyses for CENP-C ${ }^{\mathrm{Cnp} 3}(\mathrm{~B}), \mathrm{Knl} 1^{\mathrm{Spc7}}(\mathrm{C})$ levels at $c c 2, c c 1 / 3$ and act1 in wt cens strain carrying endogenous cen2-cc2 or cen2-cc2 $::$ cc1 strain transformed with pHcc2. \% IP levels in S. pombe were normalized to \%IP of central core from spiked-in S. octosporus chromatin in (B). qChIP results in (C) were reported as \%IP. Data are mean $\pm S D(n=3)$. ns, no significance (Unpaired t-test). 
A

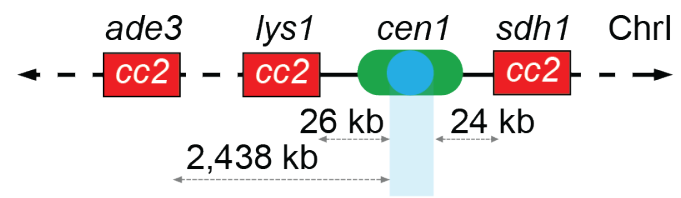

qChIP: CENP-A ${ }^{\text {Cnp1 }}$

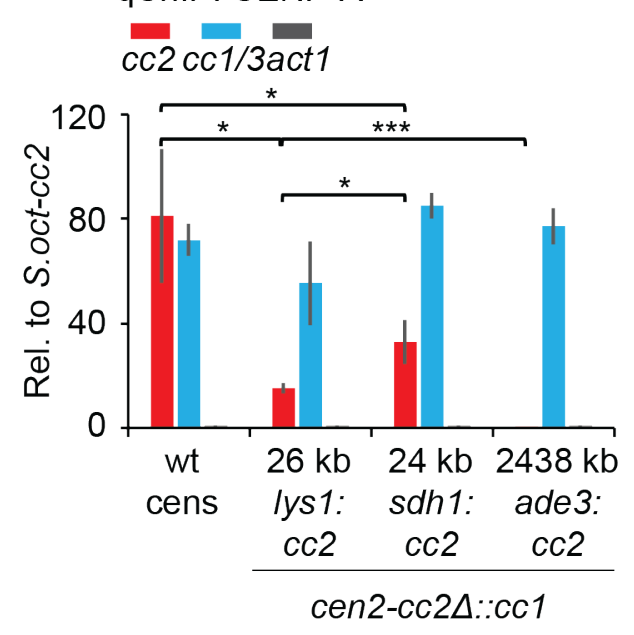

C
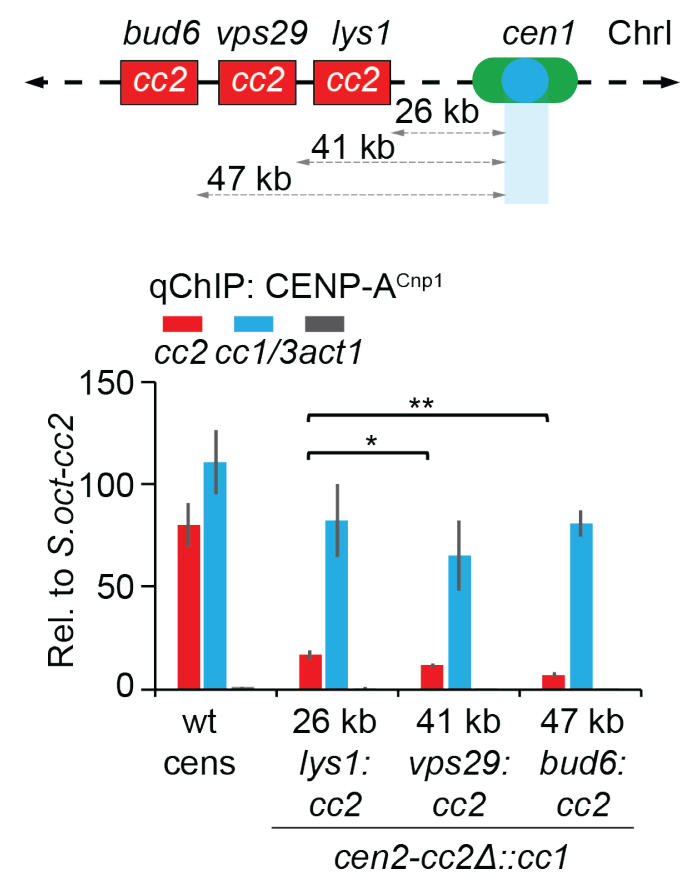

B

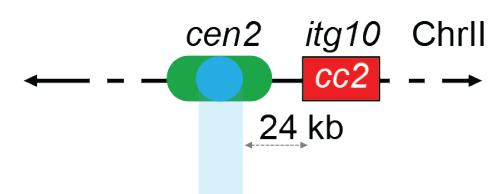

qChIP: CENP-A ${ }^{\text {Cnp1 }}$ cc2 cc1/3act1
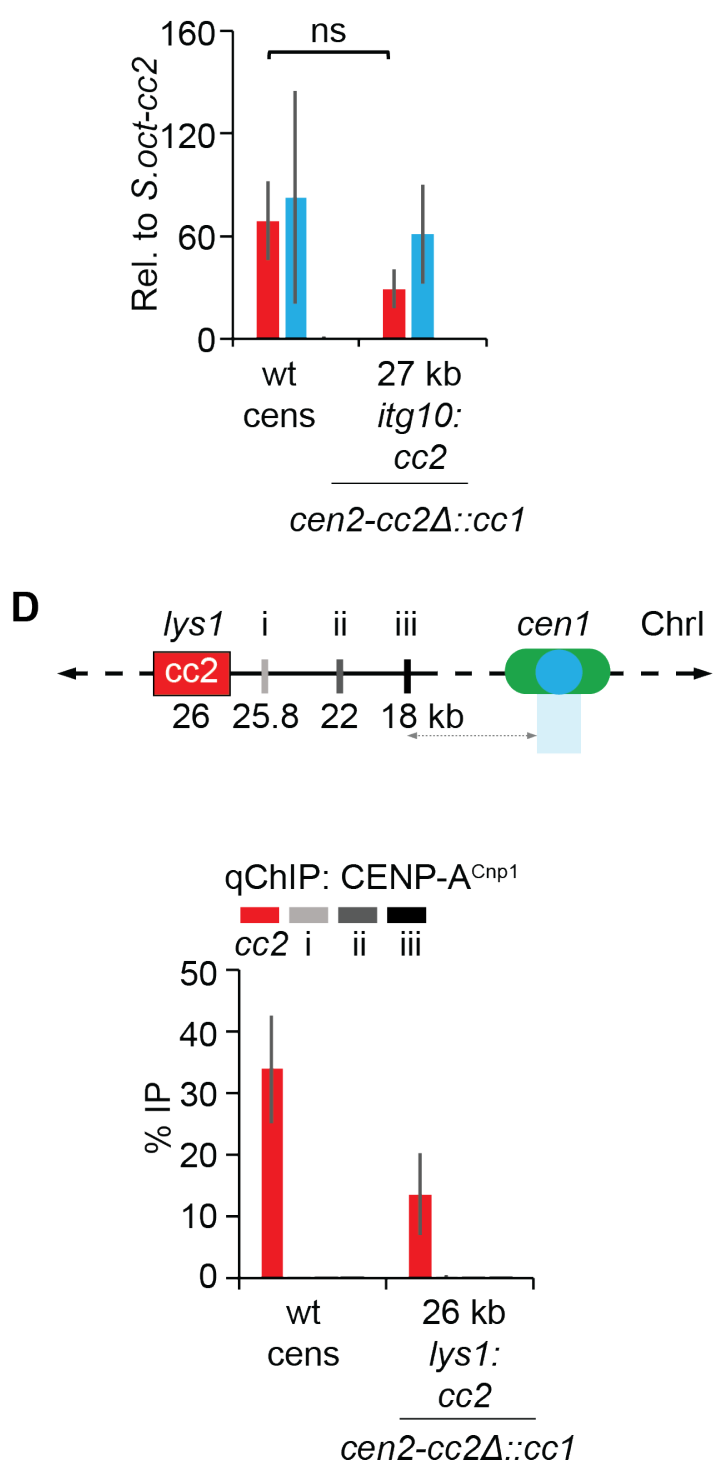
Figure S4. CENP-A ${ }^{\mathrm{Cnp} 1}$ chromatin is established on $c c 2$ inserted closed to centromeres. Relates to Figure 2

(A-C) qChIP analyses for CENP-A ${ }^{\text {Cnp1 }}$ levels at $c c 2$, cc1/3 and act1 in wt cens strain with cen2cc2 or $c c 2 \Delta:: c c 1$ strain with lys1:cc2, sdh1:cc2, ade3:cc2 (A) or itg10:cc2 (itg10; Chrll: 1,645,855$1,655,523$; B) or vps29:cc2 or bud6:cc2 (C) insertion. \%IP levels in S. pombe were normalized to \%IP of central core from spiked-in S. octosporus chromatin.

(D) qChIP analyses for CENP-A ${ }^{\text {Cnp1 }}$ levels at three euchromatic locus between lys1 and cen1: sites i, ii, iii, 25.8, 22, $18 \mathrm{~kb}$ from $c c 1$ respectively in wt cens or cc2 $::$ :cc1 strain with lys1:cc2. qChIP results were reported as \%IP. All qChIP data are mean \pm SD $(n=3)$. ns, no significance; *, $p<0.05 ;{ }^{* *}, p<0.005 ;{ }^{* *}, p<0.0005$ (Unpaired t-test). 
A

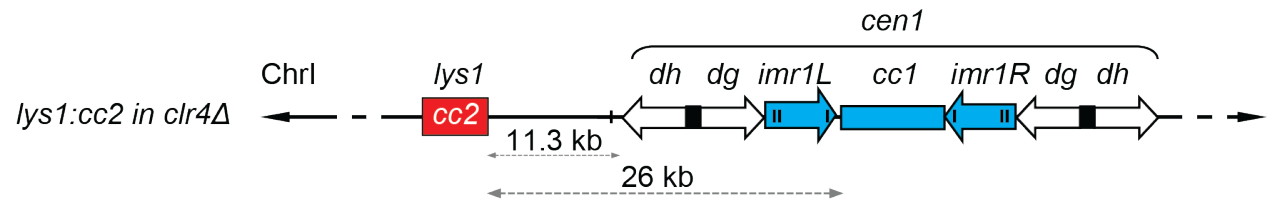

B

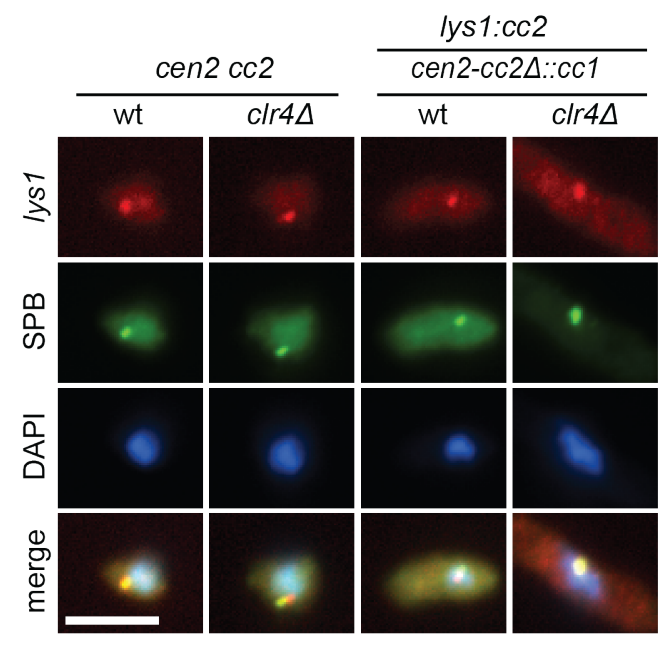

D

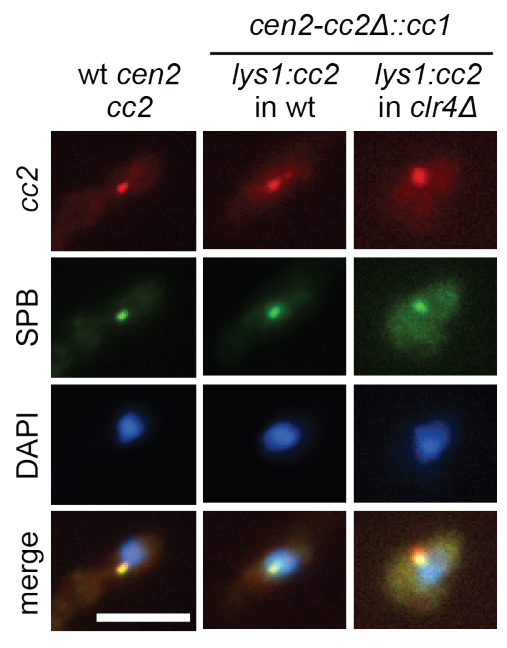

F

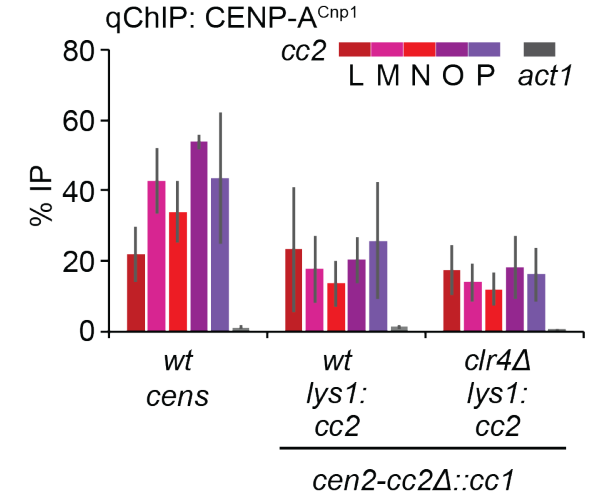

G
C lys1-SPB colocalisation

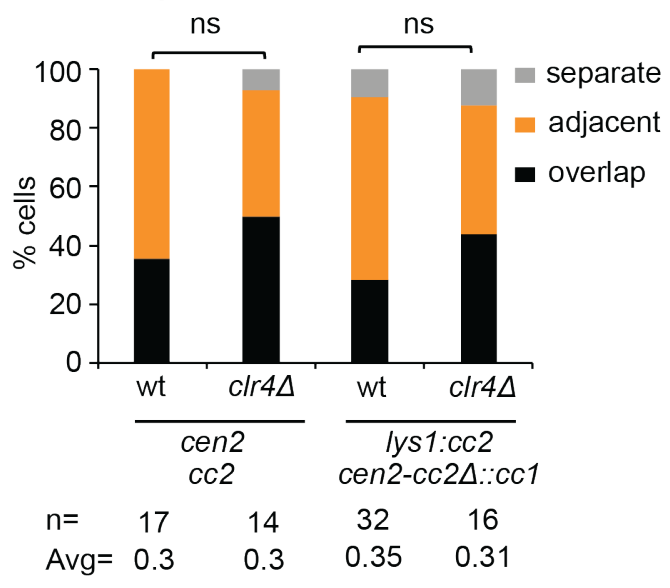

E cc2-SPB colocalisation
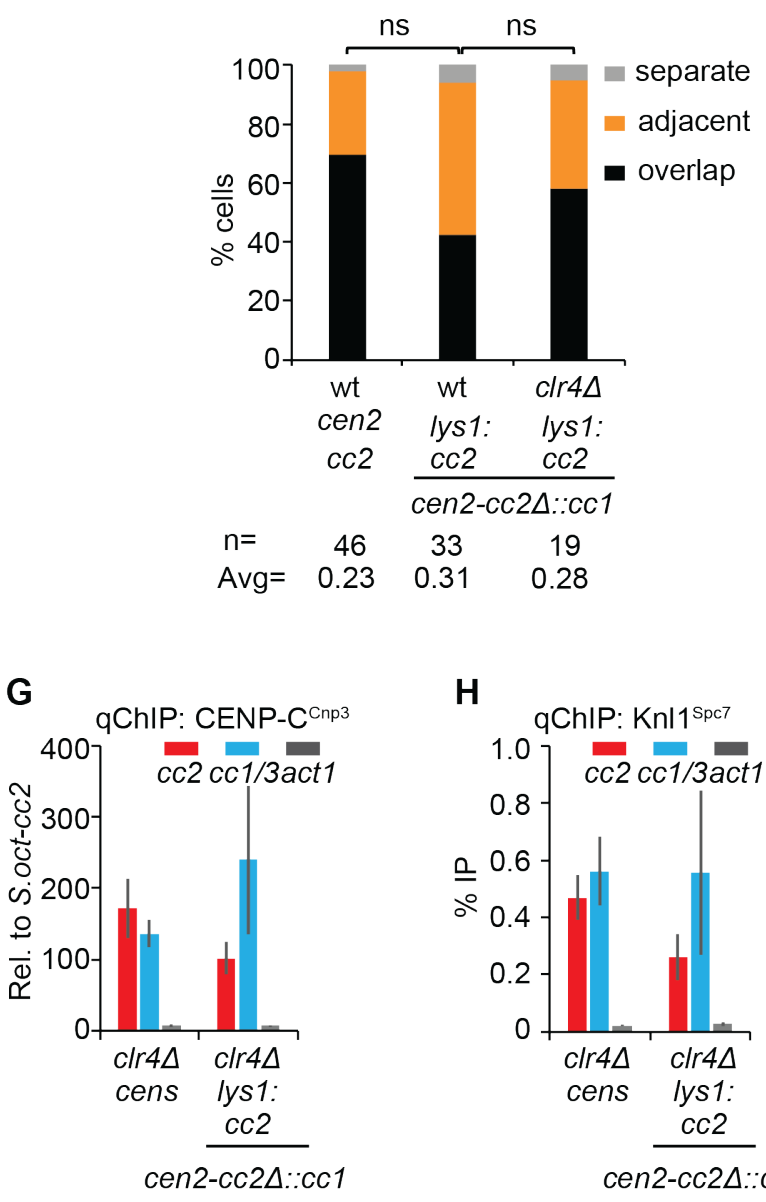

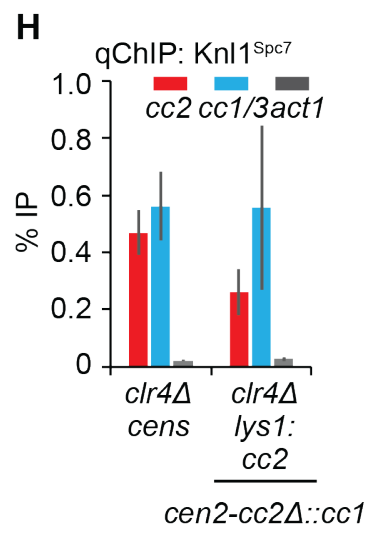


Figure S5. Centromeric heterochromatin is not required to establish CENP-A ${ }^{\text {Cnp1 }}$ chromatin on cc2 inserted close to cen1. Relates to Figure 2

(A) Diagram represents lys1:cc2 insertion, $26 \mathrm{~kb}$ or $11.3 \mathrm{~kb}$ from $c c 1$, cen1 $\mathrm{dh}$ repeat in heterochromatin-deficient $c / r 4 \Delta$ cells following transformation, respectively.

$(B, D)$ Representative images of lys1 (B) or cc2 (D) DNA FISH (red), SPB location (green; antiCdc11) and DNA staining (blue, DAPI) in wt or clr4 $\Delta$ strain with endogenous cen2-cc2 or cen2$c c 2 \Delta:: c c 1$ and $l y s 1: c c 2$. Images were scaled as in Figure 1. Scale bar, $5 \mu \mathrm{m}$.

$(C, E)$ Cells were classified into three groups according to the 3D distances between lys 1 (C) or cc2 (E) and SPB (Cdc11): overlap $(\leq 0.3 \mu \mathrm{m})$, adjacent $(0.3-0.5 \mu \mathrm{m})$ or separate $(0.5-3 \mu \mathrm{m})$. Percentage of interphase cells ( $n$, number analyzed from 3 independent experiments) in each category. Avg, average distance. ns, no significance (Mann-Whitney $U$ test).

$(\mathrm{F}-\mathrm{H})$ qChIP analyses for CENP-A ${ }^{\mathrm{Cnp} 1}(\mathrm{~F}), \mathrm{CENP}_{-} \mathrm{C}^{\mathrm{Cnp3}}(\mathrm{G}), \mathrm{Knl}^{\mathrm{Spc7}}(\mathrm{H})$ levels at $c c 2, c c 1 / 3$ and act1 wt or $c / r 4 \Delta$ strain with endogenous cen2-cc2 or cen2-cc2 $\Delta:: c c 1$ and lys1:cc2. \%IP levels in S. pombe were normalized to \%IP of central core from spiked-in S. octosporus chromatin in (G). qChIP results in $(F, H)$ were reported as \%IP. Data are mean $\pm S D(n=3)$. 
A

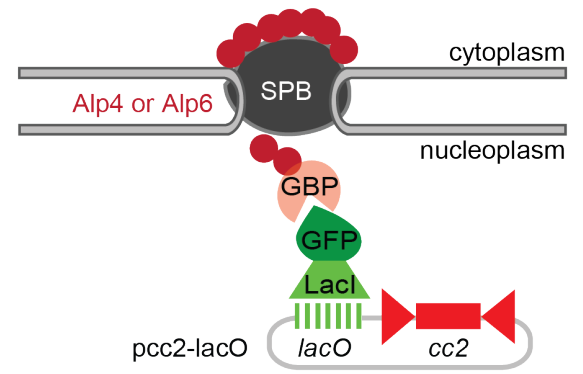

B

Lacl-GFP Alp4-GBP-mCherry merge

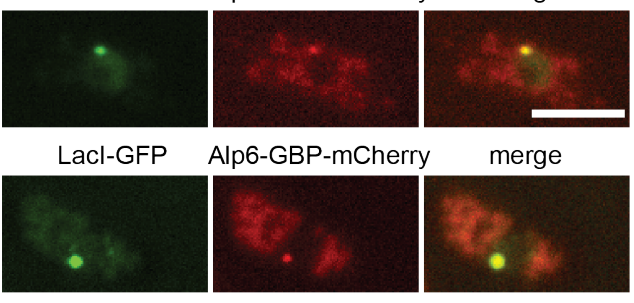

C

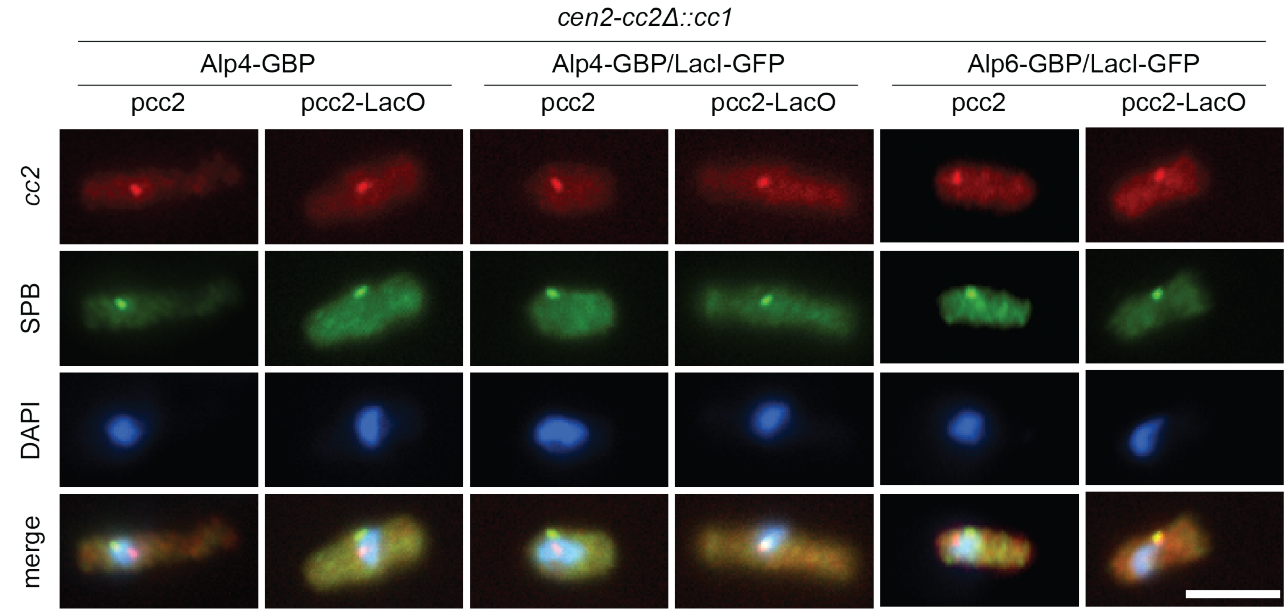

D

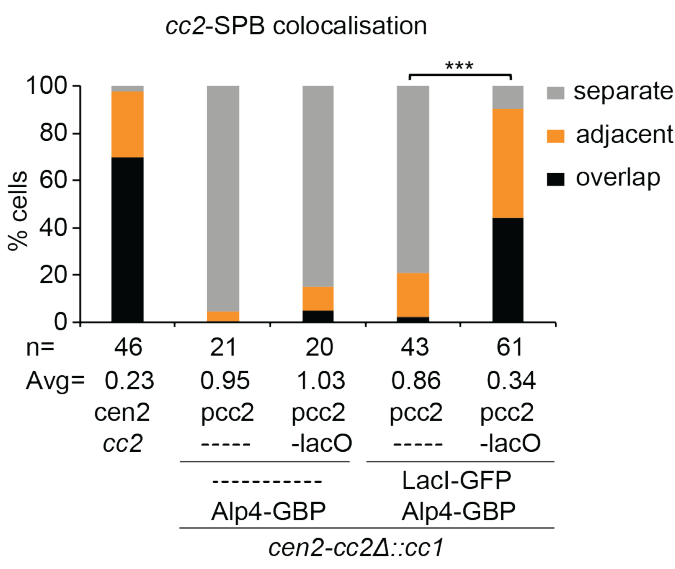

$\mathbf{F}$

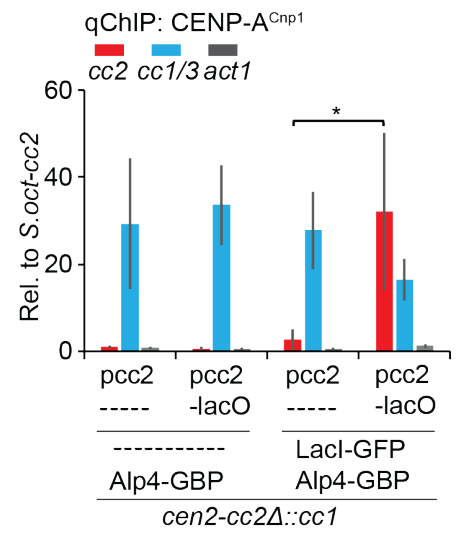

E cc2-SPB colocalisation

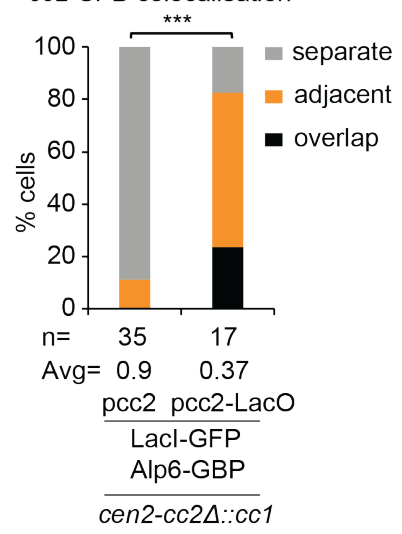

G

qChIP: CENP-A ${ }^{\text {Cnp1 }}$

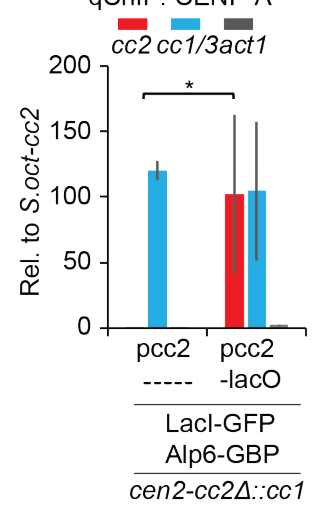


Figure S6. Tethering cc2 DNA to Alp4 or Alp6 allows CENP-A ${ }^{\mathrm{Cnp} 1}$ incorporation, Relates to Figure 5

(A) Forced association of pcc2-lacO with Alp4 or Alp6-GBP-mCherry at SPB using same tethering system as in Figure 5. A small subset of Alp4 or Alp6 molecules (red circles) are localized to the nucleoplasmic side of SPB during interphase (Bestul et al., 2017).

(B) Representative images of live cells expressing Lacl-GFP and Alp4 or Alp6-GBP-mCherry. Images were scaled as in Figure 2. Scale bar, $5 \mu \mathrm{m}$.

(C) Representative images of cc2 DNA FISH (red), SPB location (green; anti-Cdc11) and DNA staining (blue, DAPI) in indicated strains. Images were scaled as in Figure 1. Scale bar, $5 \mu \mathrm{m}$.

$(D, E)$ Cells were classified into three groups according to the 3D distances between $c c 2$ and SPB (Cdc11): overlap $(\leq 0.3 \mu \mathrm{m})$, adjacent $(0.3-0.5 \mu \mathrm{m})$ or separate $(0.5-3 \mu \mathrm{m})$. Percentage of interphase cells ( $n$, number analyzed from 3 independent experiments) in each category. Avg, average distance. ${ }^{* * *}, p<0.0001$ (Mann-Whitney $U$ test).

$(F, G)$ qChIP analyses for CENP-A ${ }^{\mathrm{Cnp} 1}$ levels at $c c 2, c c 1 / 3$ and act1 in indicated strains. \%IP levels in $S$. pombe were normalized to \%IP of central core from spiked-in $S$. octosporus chromatin. Data are mean \pm SD $(n=3-4) .{ }^{*}, p<0.05$ (Unpaired t-test). 
A

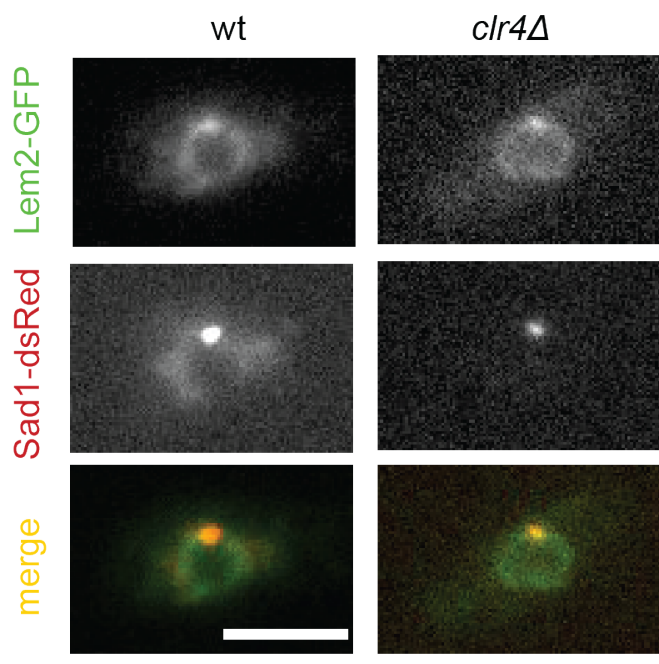

B

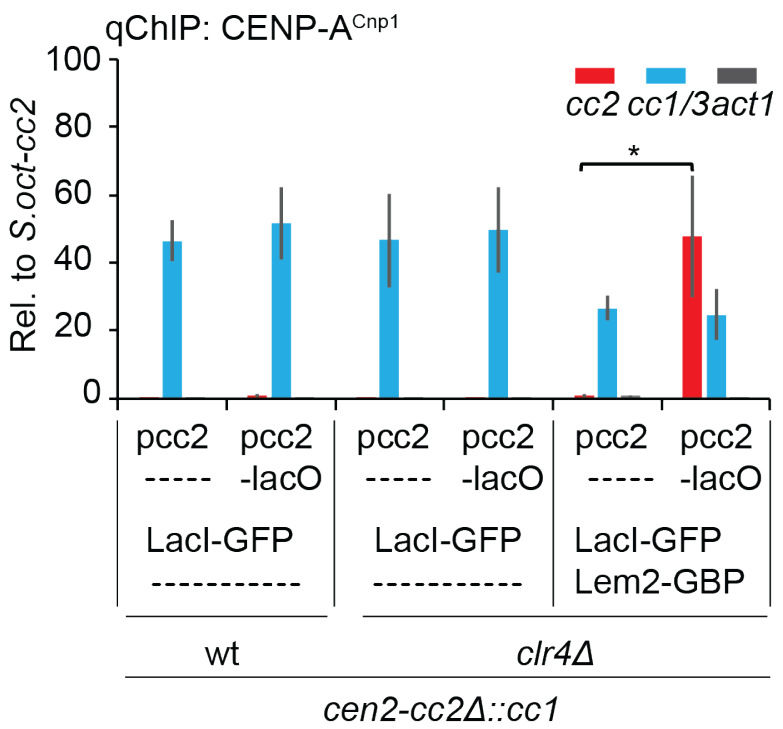

Figure S7. Tethering cc2 DNA to Lem2 allows CENP-A ${ }^{\text {Cnp1 }}$ incorporation independently of heterochromatin. Relates to Figure 5

(A) Representative images of live wt or clr4 $\Delta$ cells expressing Lem2-GFP and Sad1-dsRed. Images were scaled as in Figure 2. Scale bar, $5 \mu \mathrm{m}$.

(B) qChIP analyses for CENP-A ${ }^{\text {Cnp } 1}$ levels at $c c 2, c c 1 / 3$ and act1 in wt or clr4 $\Delta$ strains carrying cen2-cc2 $\triangle:: c c 1$ and expressing Lacl-GFP or both Lacl-GFP and Lem2-GBP-mCherry transformed with pcc2 or pcc2-lacO. \%IP levels in S. pombe were normalized to \%IP of central core from spiked-in S. octosporus chromatin. Data are mean $\pm S D(n=3) .{ }^{*}, p<0.05$ (Unpaired ttest). 
Table S1. Fission yeast strains used in this study. Related to STAR Methods.

\begin{tabular}{|c|c|c|}
\hline $\begin{array}{l}\text { Strain } \\
\text { number }\end{array}$ & Genotype & abbreviated as \\
\hline A7374 & $h+$ ade6-704-HYGMX6 his3-D1 leu1-32 ura4-DSE/D18? arg3? cc2D6kb:cc1 & wt cc2 $2:: c c 1$ \\
\hline B6596 & $h+c l r 4 \Delta$ ade6-704-HYGMX6 his3-D1 leu1-32 ura4-DSE/D18? arg3? cc2D6kb:cc1 & clr4 \\
\hline B3665 & h+lys1(=cen1):IacO his7+:lac/GFP, Sad1-dsRed-LEU2+\#1 & lys 1:lacO \\
\hline B3672 & \begin{tabular}{|l} 
h? Sad1-dsRed-LEU2+ his7+::Iacl-GFP ade3[::kanR-ura4+-lacOp] ade6-704-hygMX6 ura4- \\
DSE/D18 leu1-32? his3-D1? arg3-D4? Lys1-131?\#1
\end{tabular} & ade3:lacO \\
\hline A1741 & h- ade6-704-HYGMX6 his3-D1 leu1-32 ura4-DSE & wt cen2 \\
\hline B2950 & $\begin{array}{l}\text { h- lys 1 } \triangle 500:: c c 2-K A N M X 6 \text { ade6-704-HYGMX6 his3-D1 leu1-32 ura4-DSE/D18? arg3? } \\
\text { cc2D6kb:cc1\#1 }\end{array}$ & $\begin{array}{l}\text { lys1:cc2 \#1 or } \\
\text { lys1:cc2 in wt }\end{array}$ \\
\hline B2951 & $\begin{array}{l}\text { h- lys 1 } 1500:: c c 2-K A N M X 6 \text { ade6-704-HYGMX6 his3-D1 leu1-32 ura4-DSE/D18? arg3? } \\
\text { cc2D6kb:cc1\#2 }\end{array}$ & |ys1:cc2 \#2 \\
\hline B2953 & $\begin{array}{l}\text { h- ade3 } \triangle 500:: c c 2-K A N M X 6 \text { ade6-704-HYGMX6 his3-D1 leu1-32 ura4-DSE/D18? arg3? } \\
\text { cc2D6kb:cc1\#1 }\end{array}$ & ade3:cc2 \#1 \\
\hline B2954 & $\begin{array}{l}\text { h- ade3 } \triangle 500:: c c 2-K A N M X 6 \text { ade6-704-HYGMX6 his3-D1 leu1-32 ura4-DSE/D18? arg3? } \\
\text { cc2D6kb:cc1\#2 }\end{array}$ & ade3:cc2 \#2 \\
\hline A7373 & h- ade6-704-HYGMX6 his3-D1 leu1-32 ura4-DSE/D18? arg3? cc2D6kb:cc1 & wt cen1 or wt \\
\hline A9792 & $h$ - leu1 ura4 $\Delta c e n 1:: p A D H 1-$ loxP-KanR::ura4+cd60 (neo1R) & cen1 1 neo1R \\
\hline B3269 & $\begin{array}{l}\text { h? lys 1 } 4500:: c c 2-K A N M X 6 \text { ade6-704-HYGMX6 cc2D6kb:cc1 cen1A::pADH1-loxP-KanR::ura4+ } \\
\text { cd60 (neo1R) his3-D1/His+? leu1-32 ura4?/ura4-DSE/D18? arg3? }\end{array}$ & Iys1:cc2 in neo1R \\
\hline B3260 & $\begin{array}{l}\text { h- Chrl 5,435,010-5,435,2374226bp:::cc2-KANMX6 cen14::pADH1-loxP-KanR::ura4+ cd60 } \\
\text { (neo1R) ade6-704-HYGMX6 cc2D6kb:cc1 his3-D1 leu1-32 ura4-DSE/D18? arg3? }\end{array}$ & itg6:cc2 in neo1R \\
\hline B3265 & 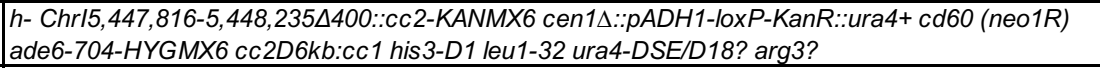 & itg7:cc2 in neo1R \\
\hline B3565 & $\begin{array}{l}\text { h- Chrl5,501,647-5,502,134D528:::cc2-KANMX6 cen14::pADH1-loxP-KanR::ura4+ cd60 (neo1R) } \\
\text { ade6-704-HYGMX6 cc2D6kb:cc1 his3-D1 leu1-32 ura4-DSE/D18? arg3? }\end{array}$ & itg8:cc2 in neo1R \\
\hline B3561 & $\begin{array}{l}\text { h- Chrl5,501,647-5,502,1344528::cc2-KANMX6 ade6-704-HYGMX6 his3-D1 leu1-32 ura4- } \\
\text { DSE/D18? arg3? cc2D6kb:cc1 }\end{array}$ & itg8:cc2 in wt cen1 \\
\hline B4295 & $h+$ Lem2-GFP-Nat, Sad1-dsRed-LEU2+ ade6-210 ura4-D18 & Lem2-GFP \\
\hline B3437 & h+ his7+:lacl-GFP cc2D6kb:cc1 ura4-DSE/D18? leu1-32 ade6-DNN arg3? his3+ & Lacl-GFP \\
\hline B4246 & $\begin{array}{l}\text { h+ Lem2-GBP-mcherry-Hyg Sad1-dsRed-LEU2+ cc2D6kb:cc1 ade6-210 his3-D1? leu1-32? ura4- } \\
\text { DSE/D18? arg3? }\end{array}$ & Lem2-GBP \\
\hline B4216 & $\begin{array}{l}\text { h+ Lem2-GBP-mcherry-Hyg his7+:lacl-GFP cc2D6kb:cc1 ura4-DSE/D18? leu1-32 ade6-DNN } \\
\text { arg3? his3+ }\end{array}$ & Lacl-GFP Lem2-GBP \\
\hline B4339 & $\begin{array}{l}\text { h- Lem2-GFP-Nat Sad1-dsRed-LEU2+ Csi1::Kan cc2_6kb:cc1 ade6-210 ura4-D18 his3-D1? leu1- } \\
32\end{array}$ & Lem2-GFP csi1 $\Delta$ \\
\hline B4866 & $\begin{array}{l}\text { h? Lem2-GBP-mcherry-Hyg, his7+:lacl-GFP csi1 }:: \text { KanMX cc2D6kb:cc1 ade6-DNN his3-D1/ } \\
\text { his3+? leu1-32 ura4-DSE/D18? arg3? }\end{array}$ & $\begin{array}{l}\text { Lacl-GFP Lem2-GBP } \\
\text { csi1 } \Delta\end{array}$ \\
\hline B4865 & $\begin{array}{l}\text { h? Lem2-GBP-mcherry-Hyg csi14::KanMX cc2D6kb:cc1 ade6-DNN his3-D1/his3+? leu1-32 ura4- } \\
\text { DSE/D18? arg3? }\end{array}$ & Lem2-GBP csi1 $\Delta$ \\
\hline B4906 & $\begin{array}{l}\text { h? Sad1-dsRed-LEU2+ his7+::lacl-GFP Csi1::Kan cc2 } 26 \mathrm{~kb}: c c 1 \text { ade6-704-hygMX6 ura4-DSE/D18 } \\
\text { leu1-32 his3-D1? arg3-D4? }\end{array}$ & Lacl-GFP csi1 $\Delta$ \\
\hline B3633 & $h+c s i 1 \Delta:: K a n M X$ cc2 $26 \mathrm{~kb}: c c 1$ ade6-704-HYGMX6 his3-D1 leu1-32 ura4-DSE/D18? arg3? & $c \operatorname{si1} \Delta$ \\
\hline B3367 & $\begin{array}{l}\text { h- sdh1 } 13344:: c c 2-K A N M X 6 \text { ade6-704-HYGMX6 his3-D1 leu1-32 ura4-DSE/D18? arg3? } \\
\text { cc2D6kb:cc1 }\end{array}$ & sdh1:cc2 \\
\hline B3639 & $\begin{array}{l}\text { h- SPBC21B10.09-10.08c } 6650:: c c 2-K A N M X 6 \text { ade6-704-HYGMX6 his3-D1 leu1-32 ura4- } \\
\text { DSE/D18? arg3? cc2D6kb:cc1 }\end{array}$ & itg10:cc2 \\
\hline B3211 & $\begin{array}{l}\text { h- SPAC15E1.02cD158bp::cc2-KANMX6 ade6-704-HYGMX6 his3-D1 leu1-32 ura4-DSE/D18? } \\
\text { arg3? cc2D6kb:cc1 }\end{array}$ & bud6:cc2 \\
\hline B3297 & $\begin{array}{l}\text { h- vps294154::cc2-KANMX6 ade6-704-HYGMX6 his3-D1 leu1-32 ura4-DSE/D18? arg3? } \\
\text { cc2D6kb:cc1 }\end{array}$ & vps29:cc2 \\
\hline A7255 & $h+c l r 4:: N A T$ ade6-210 his3-D1 leu1-32 ura4-D18 arg3-D4 cc2D6kb:cc1 & $c / r 4 \Delta c c 2 \Delta:: c c 1$ \\
\hline B3257 & $h+$ clr4::NAT lys 1 $5500:: c c 2-K A N M X 6$ ade6-210 his3-D1 leu1-32 ura4-D18 arg3-D4 cc2D6kb:cc1 & Iys 1:cc2 in clr4 4 \\
\hline 8946 & h- clr4:NAT ade6-210 leu1-32 ura4-D18 arg3-D his3D & clr4 $\Delta$ cen 2 cc2 \\
\hline B5560 & $\begin{array}{l}\text { h? cc2 } 26 \text { kb:cc1 Alp4-GBP-mcherry-Hyg his7+:lacl-GFP leu1-32 ura4-D18/DS-E ade6- } \\
\text { 704::hphMX/ade6-DNN? his3? arg3? }\end{array}$ & Lacl-GFP Alp4-GBP \\
\hline B5564 & $\begin{array}{l}\text { h? cc2 } 26 \text { kb:cc1 Alp4-GBP-mcherry-Hyg leu1-32 ura4-D18/DS-E ade6-704::hphMX/ade6-DNN? } \\
\text { his3? arg3? }\end{array}$ & Alp4-GBP \\
\hline B5572 & $\begin{array}{l}\text { h? cc2 } 26 \mathrm{~kb}: c c 1 \text { Alp6-GBP-mcherry-Hyg his 7+:lacl-GFP leu1-32 ura4-D18/DS-E ade6- } \\
\text { 704::hphMX/ade6-DNN? his3? arg3? \#8 }\end{array}$ & Lacl-GFP Alp6-GBP \\
\hline B4349 & $\begin{array}{l}\text { h- lem2-GBP-mcherry-Hyg, his 7+:lacl-GFP, clr4::NAT, cc2D6kb:cc1 ura4-DSE/D18? his3+/his3- } \\
\text { D1? arg3? leu1-32 ade6-DNN/ade6? }\end{array}$ & $\begin{array}{l}\text { Lacl-GFP Lem2-GBP } \\
\text { clr4 }\end{array}$ \\
\hline B4351 & $\begin{array}{l}\text { h? his7+:lacl-GFP clr4::NAT cc2D6kb:cc1 ura4-DSE/D18? his3+/his3-D1? arg3? leu1-32 ade6- } \\
\text { DNN/ade6? }\end{array}$ & Lacl-GFP clr4 $\Delta$ \\
\hline B4343 & h- Iem2-GFP-Nat Sad1-dsRed-LEU2+ clr4::hph+ ade6-210 ura4-D18 his3-D1? leu1-32 & Lem2-GFP clr4 $\Delta$ \\
\hline
\end{tabular}


Table S2. Plasmids used in this study. Related to STAR Methods.

\begin{tabular}{|c|c|c|c|c|c|c|c|c|c|c|}
\hline \multirow{2}{*}{$\begin{array}{l}\text { Name used in main } \\
\text { text (if appicable) }\end{array}$} & \multirow[t]{2}{*}{ Name } & \multicolumn{7}{|c|}{ Plasmid features } & \multirow[t]{2}{*}{ Notes } & \multirow[t]{2}{*}{ Resource } \\
\hline & & marker 1 & marker 2 & marker 3 & $\begin{array}{l}\text { central } \\
\text { core }\end{array}$ & \begin{tabular}{|l|}
$K^{\prime \prime}$ \\
repeats
\end{tabular} & laco & \begin{tabular}{|l|}
$\begin{array}{l}\text { Other } \\
\text { features }\end{array}$ \\
\end{tabular} & & \\
\hline & pMC52 & & & kan & & & & & $\begin{array}{l}\text { Used for ectopic } c c 2 \text { insertion } \\
\text { and } c c 2 \text { FISH probe }\end{array}$ & This study \\
\hline pcc2 & pMC2 (pcc2) & ura4 & sup3e & kan & $8.6 \mathrm{~kb} \mathrm{cc2}$ & & & & Used for tethering assay & This study \\
\hline pcc2-lacO & pMC12 (pcc2-lacO) & ura4 & sup3e & kan & $8.6 \mathrm{~kb} c c 2$ & & $\begin{array}{l}2.8 \mathrm{~kb} ; \sim 90 \\
\text { lacO } \text { sites }\end{array}$ & & Used for tethering assay & This study \\
\hline pHcc2 & pHcc2 & ura4 & sup3e & & $8.6 \mathrm{~kb} \mathrm{cc} 2$ & $5.6 \mathrm{~kb} K^{\prime \prime}$ & & & $\begin{array}{l}\text { Used for minichromosome } \\
\text { establishment assay }\end{array}$ & This study \\
\hline \multirow[t]{6}{*}{ pHet } & pMC183 (pHet) & & & nat & & $2 \mathrm{~kb} K^{\prime \prime}$ & & & $\begin{array}{l}\text { Used to check centromeric } \\
\text { heterochromatin nuclear } \\
\text { localization }\end{array}$ & This study \\
\hline & pMC1 & ura4 & sup3e & kan & & & & & $\begin{array}{l}\text { Used for plasmid backbone } \\
\text { FISH probe }\end{array}$ & This study \\
\hline & pLSB-Kan & & & kan & & & & Cas9 & Used to make $c / r 4 \Delta$ mutant & $\begin{array}{l}\text { (Torres-Garcia et } \\
\text { al., 2020). }\end{array}$ \\
\hline & clr4-pLSB-Kan & & & kan & & & & $\begin{array}{l}\text { Cas9 and clr4 } \\
\text { sgRNA }\end{array}$ & Used to make $c / r 4 \Delta$ mutant & This study \\
\hline & $\begin{array}{l}\text { pFA6a-GBP-mCherry- } \\
\text { hygMX6 }\end{array}$ & & & hyg & & & & $\begin{array}{l}\text { GBP and } \\
\text { mCherry }\end{array}$ & $\begin{array}{l}\text { Used to make Lem2/Alp4/Alp6 } \\
\text { GBP-mCherry-Hgy fusion } \\
\text { protein }\end{array}$ & \begin{tabular}{|l} 
Gift from Julia \\
Promisel Cooper \\
(Fernández-Alvarez \\
et al., 2016)
\end{tabular} \\
\hline & pFA6a-GFP-NatMX6 & & & nat & & & & GFP & $\begin{array}{l}\text { Used to make Lem2-GFP } \\
\text { fusion protein }\end{array}$ & This study \\
\hline
\end{tabular}


Table S3. Primers used in this study. Related to STAR Methods.

\begin{tabular}{|c|c|}
\hline & PCR primers \\
\hline Name & Sequence 5'-3' \\
\hline WW1-lys1-R-Sacl & TACTACGAGCTCATGGTCTGAGAGACTTAAGAG \\
\hline WW2-lys1-F-Mscl & TACTACTGGCCAATCTAATCCCGTGGTAGAAAG \\
\hline WW3-lys1-Xhol & TACTACCTCGAGGCTCGTCAGATTATATATCTG \\
\hline WW4-lys 1-F-Kpnl & TACTACGGTACCGTAACCTTATACTTGTTAGCC \\
\hline WW5-ade3-R-Sacl & TACTACGAGCTCGATGCTTGTACAACTATGGAC \\
\hline WW6-ade3-F-Mscl & TACTACTGGCCAGTGATGAGGAAGGATTTAGAC \\
\hline WW7-ade3-R-Xhol & TACTACCTCGAGGTAAAGTTGAGGACATCGGAT \\
\hline WW8-ade3-F-Kpnl & TACTACGGTACCAGAGGTTTCATGCCAAATTCC \\
\hline WW201-sdh1-F-Kpnl & TACTACGGTACCGCACTGGTTTAACTTCATTC \\
\hline WW202-sdh1-R-Xhol & TACTACCTCGAGTTATTTGCAGCTTTCTCACC \\
\hline WW203-sdh1-F-Mscl & TACTACTGGCCACAAAATAGTCTTGTGAGCAC \\
\hline WW204-sdh1-R-Sacl & TACTACGAGCTCAAAATGGAGGGAATCTGAAG \\
\hline WW115-vps29-F-Sacl & TACTACGAGCTCCACACCACTCAACAACATC \\
\hline WW116-vps29-R-Mscl & TACTACTGGCCACGAGCTAAAATACTTAGTGC \\
\hline WW117-vps29-F-Xhol & TACTACCTCGAGTTAATGGATGTTCAAGGAGC \\
\hline WW118-vps29-R-Kpnl & TACTACGGTACCCTGACACTGGAATATAATCG \\
\hline WW095-bud6-F-Sacl & TACTACGAGCTCTTTGGCAATGATTGTATGCC \\
\hline WW096-bud6-R-Mscl & TACTACTGGCCAGAAGGTTGCTACAACTTATG \\
\hline WW097-bud6-F-Xhol & TACTACCTCGAGGTAGGATGGCATTTCTTGTA \\
\hline WW098-bud6-R-Kpnl & TACTACGGTACCTCTTATTCCTCTAGGTTAGG \\
\hline WW111-itg6-F-Sacl & TACTACGAGCTCTAAAAGCCTGTCAAATCAGG \\
\hline WW112-itg6-R-Mscl & TACTACTGGCCATCTTATTAAGTAACACGGAG \\
\hline WW113-itg6-F-Xhol & TACTACCTCGAGCCCATGCAAAGTATACTATG \\
\hline WW114-itg6-R-Kpnl & TACTACGGTACCTTAGTAGATCGTTTCCAGTG \\
\hline WW107-itg6-F-Sacl & TACTACGAGCTCAGGTGATGTTTGTACAGTAC \\
\hline WW108-itg7-R-Mscl & TACTACTGGCCAATGGTAGATATGCACCTTAC \\
\hline WW109-itg7-F-Xhol & TACTACCTCGAGTTTCAAAGATGATGCCACTG \\
\hline WW110-itg7-Kpnl & TACTACGGTACCAGATATATCCGAAGACTTCC \\
\hline WW289-itg8-F-Sacl & TACTACGAGCTCTCGCATAAGAGCTAATGTTG \\
\hline WW290-itg8-R-Mscl & TACTACTGGCCAAGGCATTATGATCTGTATCG \\
\hline WW291-itg8-F-Xhol & TACTACCTCGAGTACAATACGCAGATCATGTG \\
\hline WW292-itg8-R-Kpnl & TACTACGGTACCCCTTAACTAATTGGTGTTCG \\
\hline WW197-itg10-F-Kpnl & TACTACGGTACCAAGCTCTATATCTTGTCGAG \\
\hline WW198-itg10-R-Xhol & TACTACCTCGAGTAGCATATTATCCGTTAGCC \\
\hline WW297-itg10-F-Mscl & TACTACTGGCCACTAGTTGCTGTACTATGTAG \\
\hline WW298-itg10-R-Sacl & TACTACGAGCTCCCAACTTTACAATGCAGTTG \\
\hline WW17-lys1-F & TCCATTCTTTACGAGATGGTG \\
\hline WW18-lys1-R & ATTTGACTGTTCTGGTTTCGG \\
\hline WW23-lys1-downstream & ATACTGAGTACAATCAGCGTC \\
\hline WA519 & CGTCAAGACTGTCAAGGAG \\
\hline WA529 & AGTACGAAAACAGGAATGTG \\
\hline WW24-lys 1-upstream & ATGAAGGAATTGGCTCATCAG \\
\hline WW25-ade3-F & AGATCATCAACAGGAGAGAAG \\
\hline WW26-ade3-R & TGATCAAATGGTTGGTCCTTG \\
\hline WW31-ade3-downstream & CATCTGTTCAACGTGGTAAC \\
\hline WA519 & CGTCAAGACTGTCAAGGAG \\
\hline WW32-ade3-upstream & GGATTGTCACGAATTTCTTGC \\
\hline WA529 & AGTACGAAAACAGGAATGTG \\
\hline WW236-sdh1-check-R & ATTACCAAAGCTTCTCCTTC \\
\hline
\end{tabular}

